\title{
Ruthenium Catalyzed Intramolecular Hydroarylation of Arenes and Mechanistic Study: Synthesis of Dihydrobenzofurans, Indolines, and Chromans
}

\author{
Raja K. Rit, ${ }^{*}$ Koushik Ghosh, Rajib Mandal, and Akhila K. Sahoo* \\ School of Chemistry, University of Hyderabad, Hyderabad-500046, India
}

SUPPORTING INFORMATION 
Table of Contents

Page

X-Ray data

S3-S5

NMR data

S6-S122

Hammett Study

S123

ESI-MS data

S124 
X-ray crystallography: Single crystal X-ray data for the compound $\mathbf{2 p}$ was collected using the detector system $[\lambda(\mathrm{Mo}-\mathrm{K} \alpha)=0.71073 \AA]$ at $293 \mathrm{~K}$, graphite monochromator with a $\omega$ scan width of $0.3^{\circ}$, crystal-detector distance $60 \mathrm{~mm}$, collimator $0.5 \mathrm{~mm}$. The SMART software ${ }^{1}$ was used for the intensity data acquisition and the SAINTPLUS Software ${ }^{1}$ was used for the data extraction. In each case, absorption correction was performed with the help of SADABS program, ${ }^{1}$ an empirical absorption correction using equivalent reflections was performed with the program. The structure was solved using SHELXS- $97,{ }^{2}$ and full-matrix least-squares refinement against $\mathrm{F}^{2}$ was carried out using SHELXL-97. ${ }^{2}$ All non-hydrogen atoms were refined anisotropically. Aromatic and methyl hydrogens were introduced on calculated positions and included in the refinement riding on their respective parent atoms.

\section{X-ray crystal structure and data for $2 p$ :}

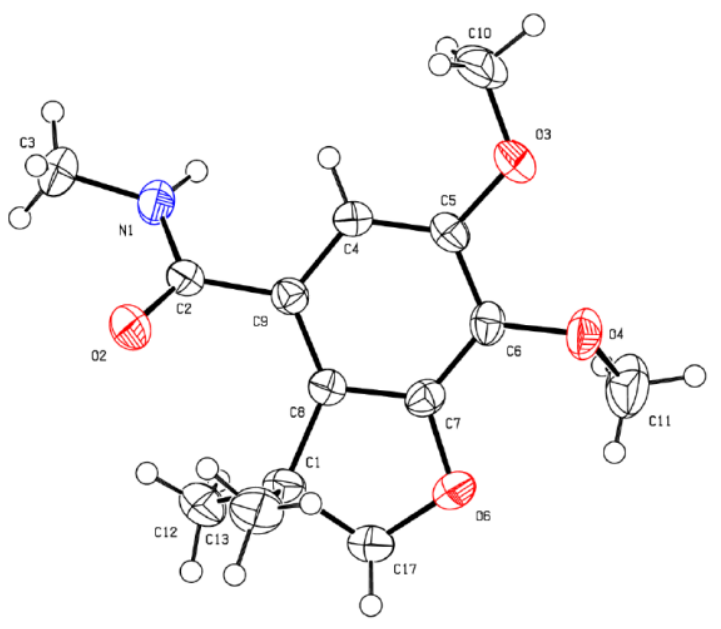

Figure 1. Thermal ellipsoidal plot of compound 2p with atom labeling scheme. Displacement ellipsoids are drawn at 50\% probability level except for the $\mathrm{H}$ atoms, which are shown as circles of arbitrary radius. 
Table 1. Crystal data for $2 p$.

\begin{tabular}{|c|c|}
\hline Compound & $2 p$ \\
\hline Formula & $\mathrm{C}_{14} \mathrm{H}_{19} \mathrm{~N} \mathrm{O}_{4}$ \\
\hline $\mathrm{M}_{\mathrm{w}}$ & 265.30 \\
\hline Crystal system & triclinic \\
\hline Space group & $\mathrm{Pbca}$ \\
\hline$T[\mathrm{~K}]$ & $293 \mathrm{~K}$ \\
\hline$a[\AA]$ & $11.1060(5)$ \\
\hline$b[\AA]$ & $10.7491(5)$ \\
\hline$c[\AA]$ & $23.3289(11)$ \\
\hline$\alpha\left[^{\circ}\right]$ & 90 \\
\hline$\beta\left[^{\circ}\right]$ & 90 \\
\hline$\gamma\left[^{\circ}\right]$ & 90 \\
\hline $\mathrm{Z}$ & 8 \\
\hline $\mathrm{V}\left[\AA^{3}\right]$ & $2785.0(2)$ \\
\hline $\mathrm{D}_{\text {calc }}\left[\mathrm{g} \mathrm{cm}^{-3}\right]$ & 1.265 \\
\hline$\mu\left[\mathrm{mm}^{-1}\right]$ & 0.093 \\
\hline Total reflns & 2519 \\
\hline Unique reflns & 1853 \\
\hline Observed reflns & 1740 \\
\hline
\end{tabular}




$$
\begin{array}{cc}
R_{1}[I>2 \sigma(I)] & 0.0377 \\
w R_{2}[\text { all }] & 0.1038 \\
\text { GOF } & 1.053
\end{array}
$$

Diffractometer Bruker D8 Quest CCD

\section{References:}

1. Bruker SMART V5.630 and SAINT-PLUS V6.45, Bruker-Nonius Analytical X-ray Systems Inc.:Madison, Wisconsin, USA 2003. SADABS, Empirical absorption correction program, Bruker AXS Inc., Madison, Wisconsin, USA 1997.

2. Sheldrick G M, Acta Crystallogr 64A (2008) 112. 


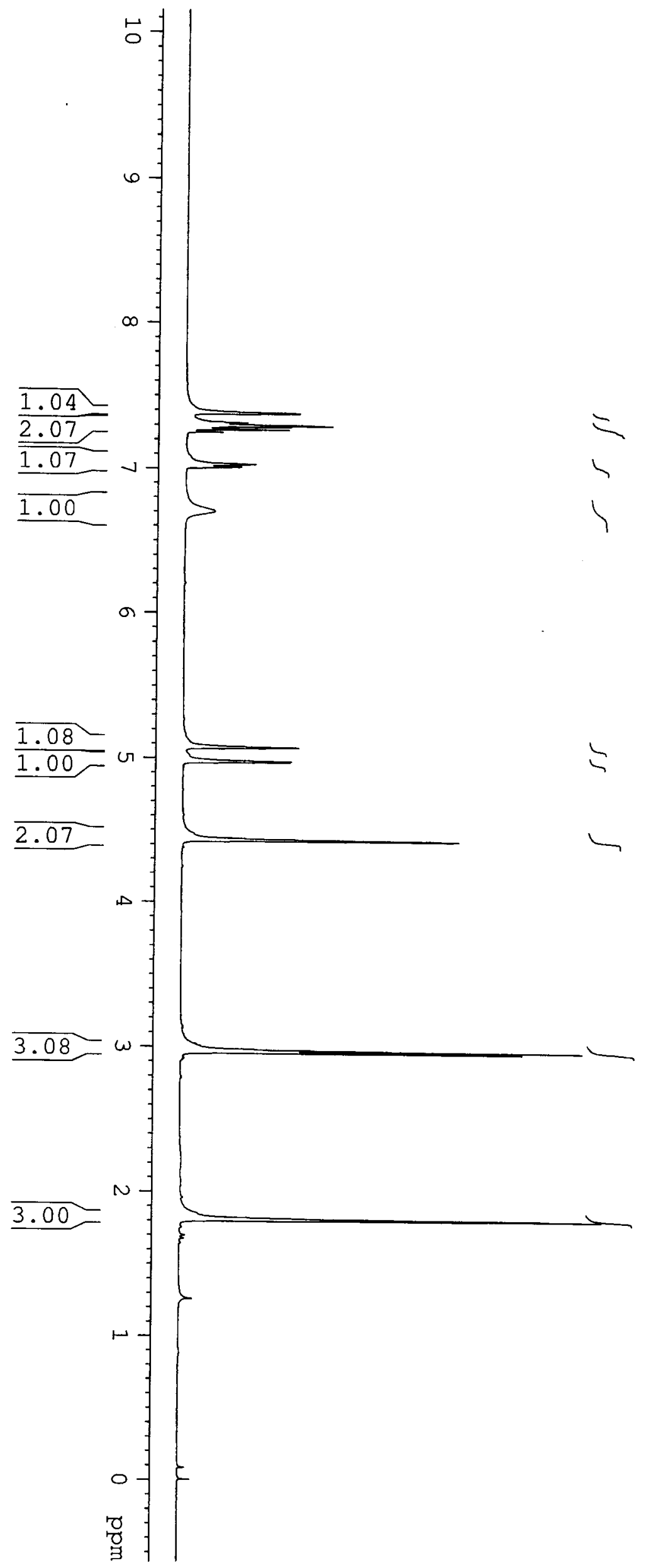

7.384

$-7.381$

7.375

7.317

7.314

7.311

7.299

7.295

7.292

7.288

$-7.281$

$-7.269$

$-7.249$

$-7.033$

7.029

7.027

7.023

7.014

7.010

7.008

4.004

6.703

$-5.074$

4.976

$-4.427$

$-2.969$

$-2.957$

$-1.803$ 

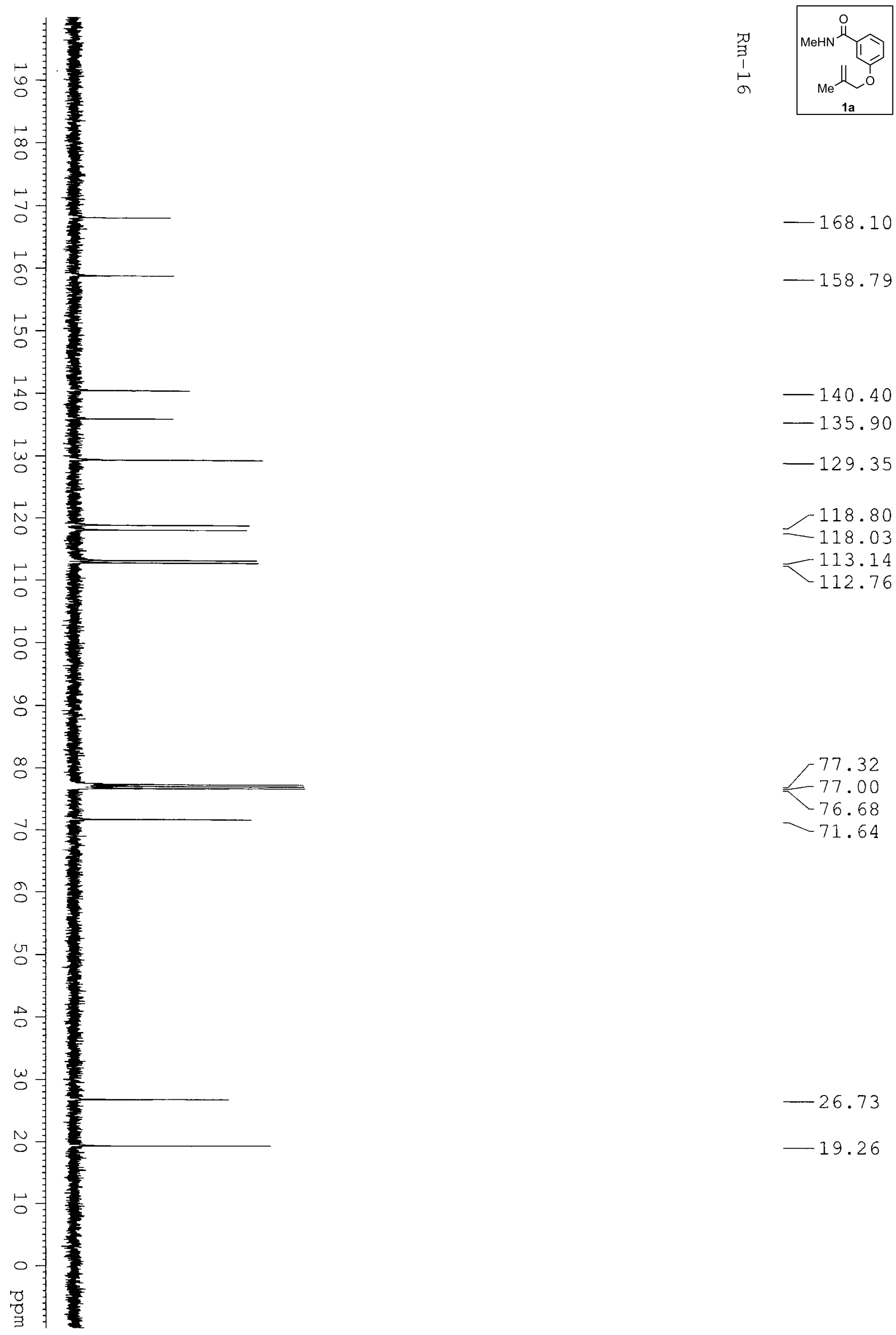

$-168.10$

$-158.79$

$-140.40$

$-135.90$

$-129.35$

$-118.80$

$-118.03$

$-113.14$

112.76

77.32

77.00
-76.68

76.68

$-71.64$

$-26.73$

$-19.26$ 


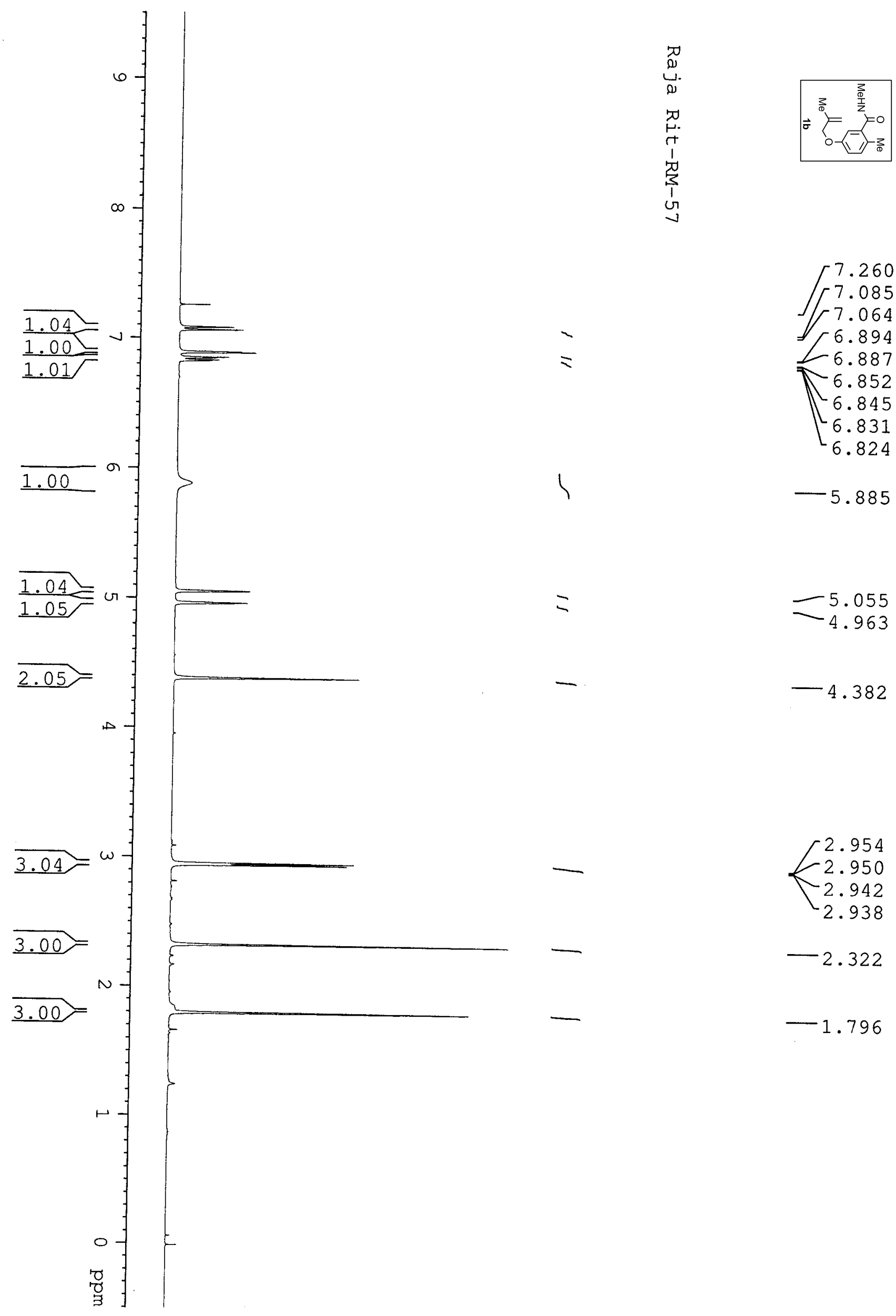




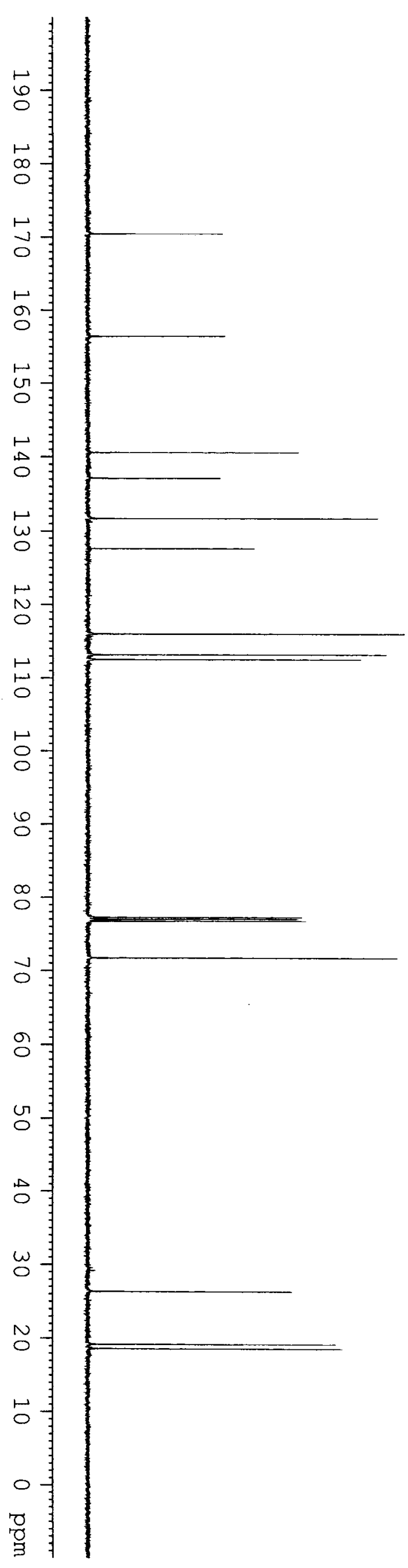

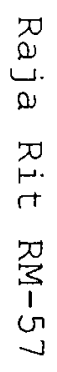

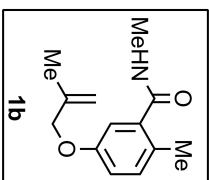

$-170.46$

$-156.44$

$-140.58$

$-137.12$

$-131.69$

$-127.61$

$-115.99$

$-113.18$

$-112.53$

77.25

77.00

76.74

$-71.72$

$-26.36$

-19.20
-18.61

$\checkmark 18.61$ 


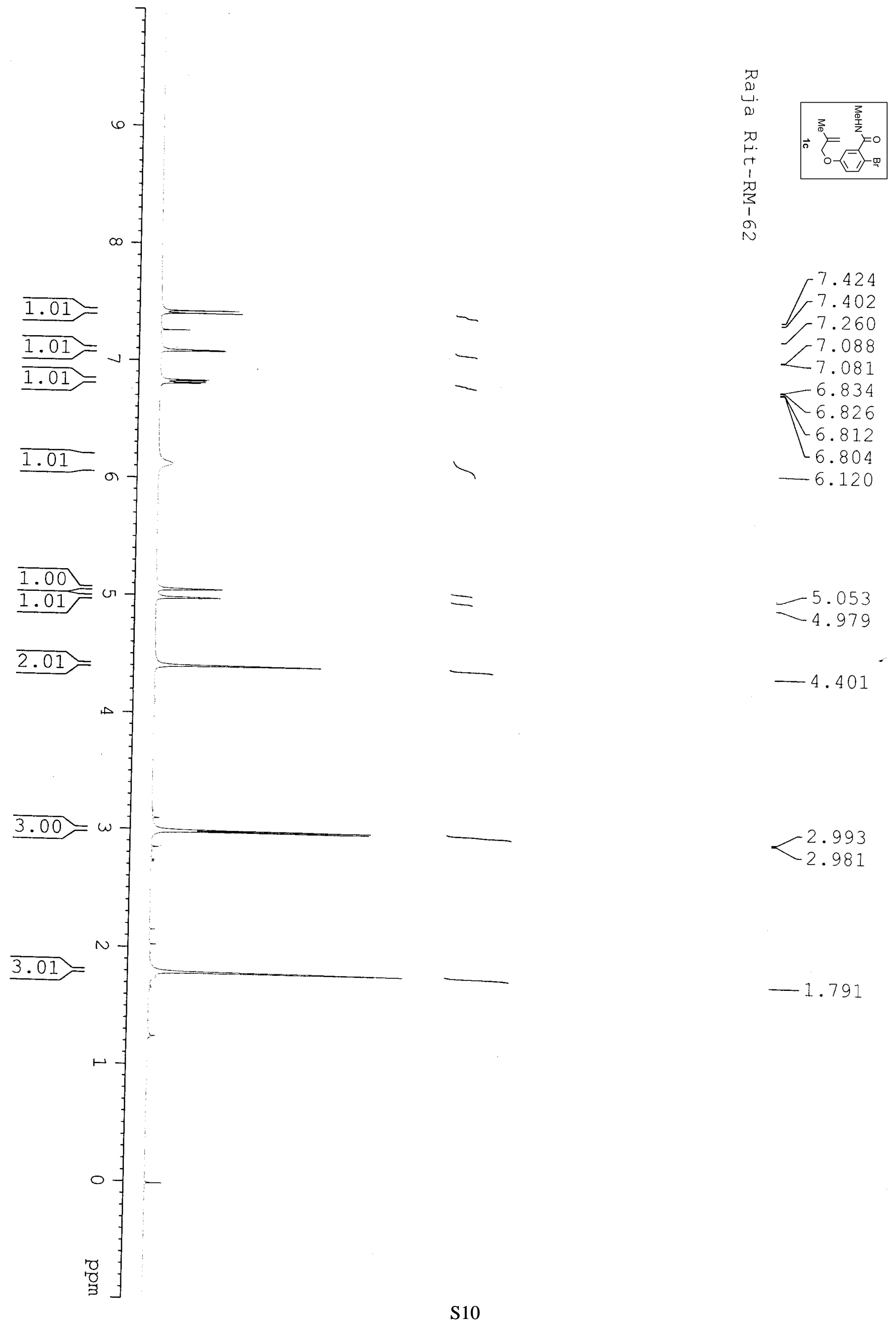



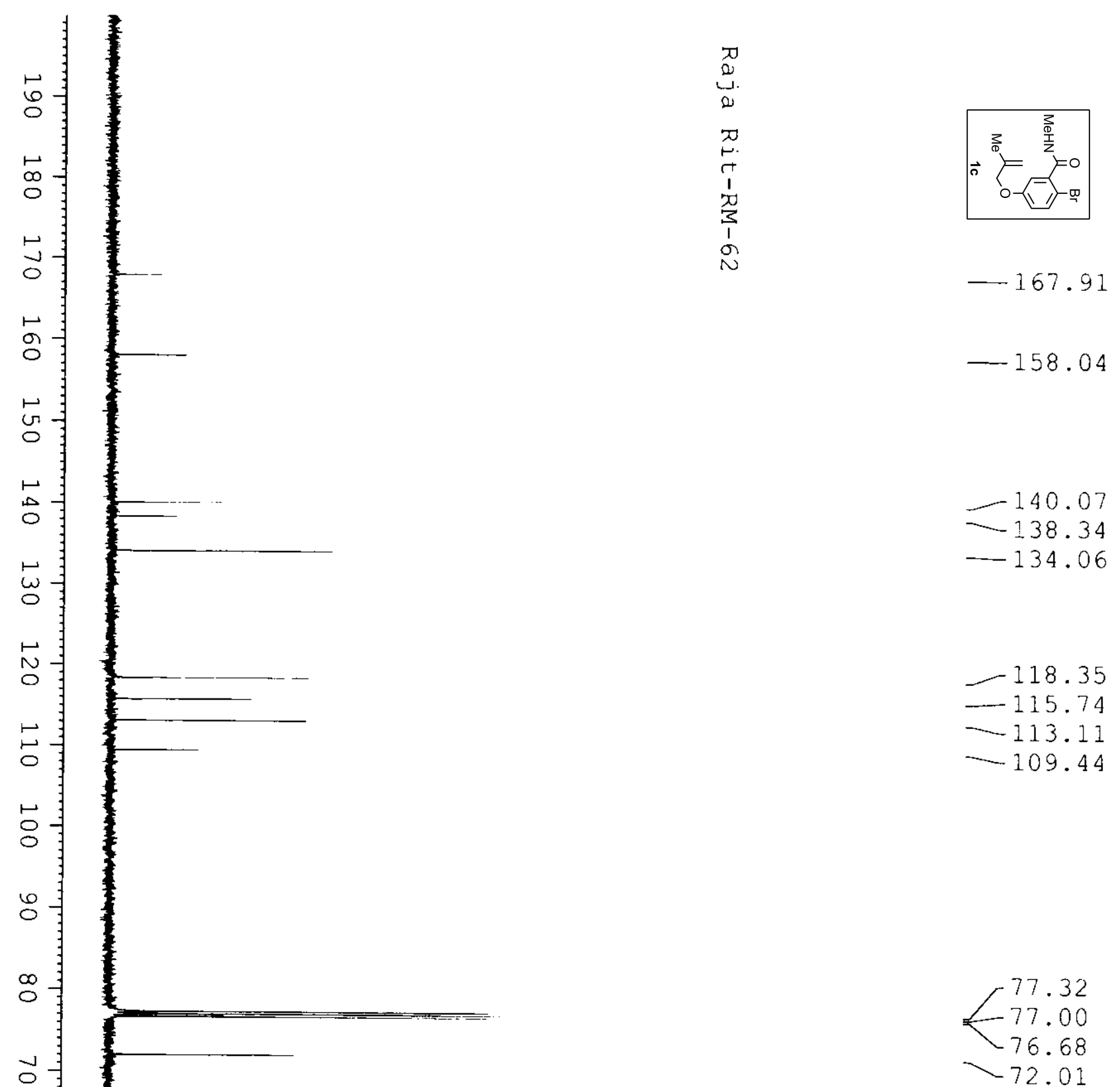

$-26.68$

$-19.25$ 


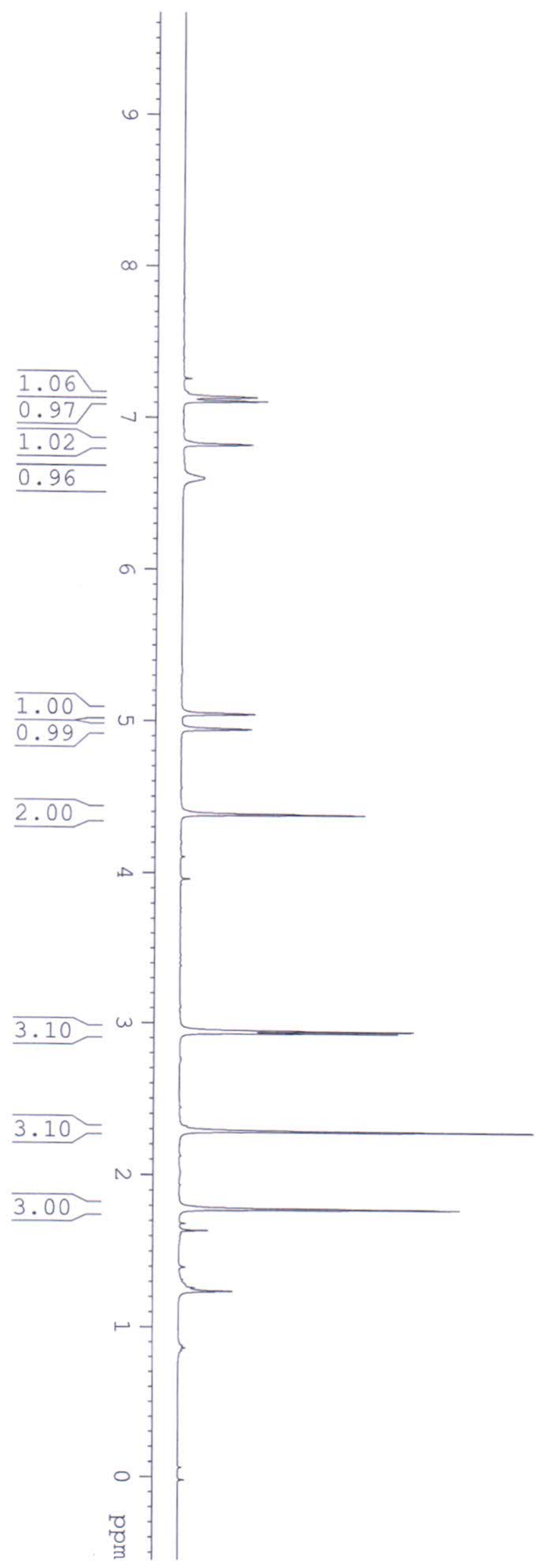

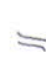

$\checkmark$

$\checkmark$

$-5.044$

$-4.944$

$-4.381$

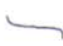

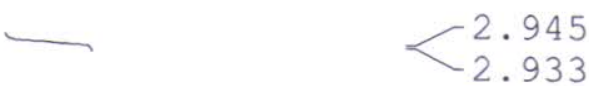

$-2.285$

$-1.778$ 

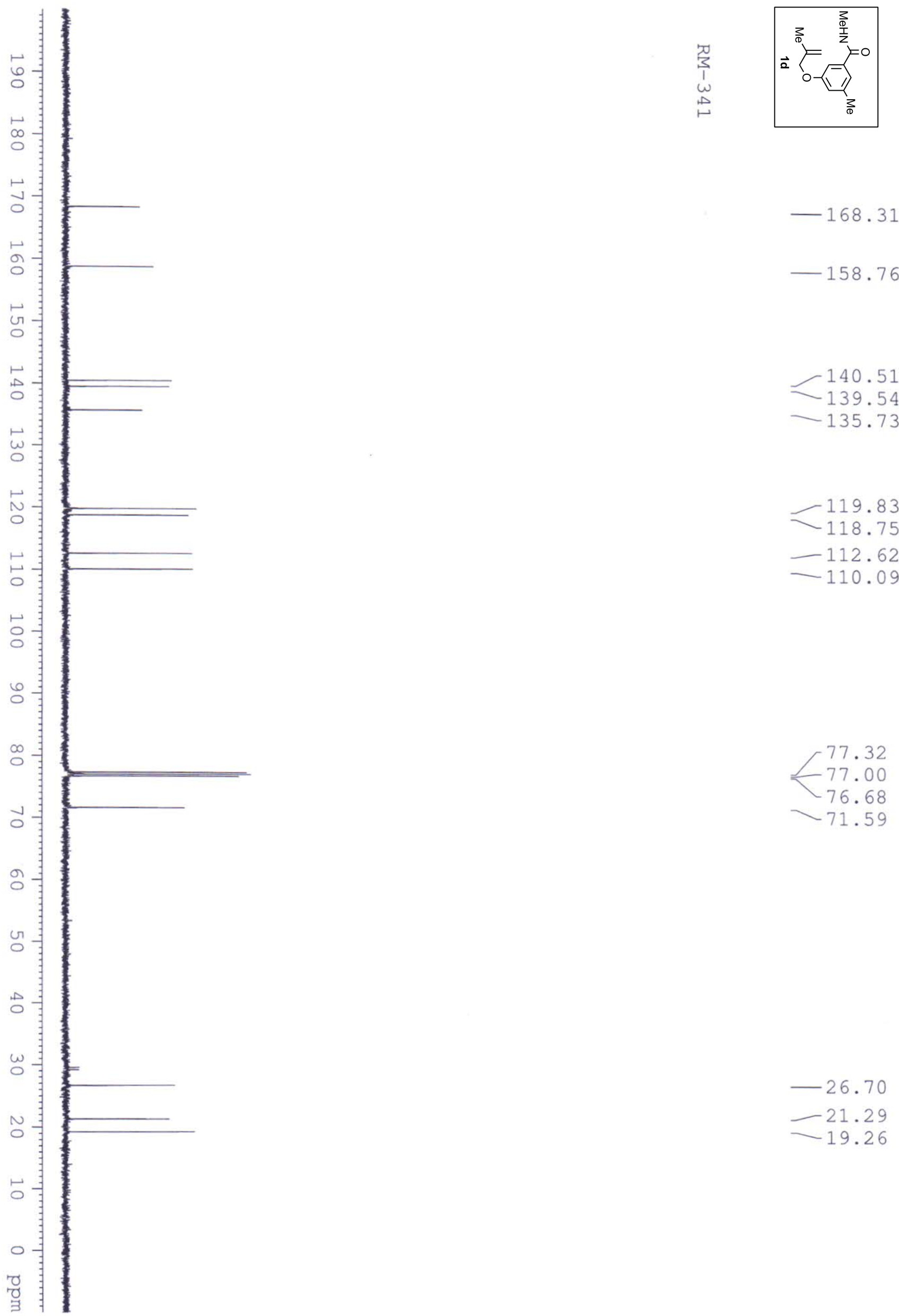

$-158.76$
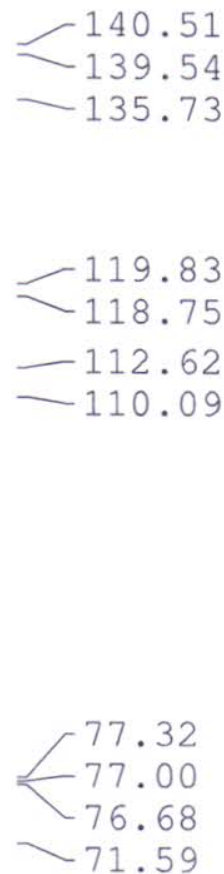

-26.70
-21.29
-19.26 

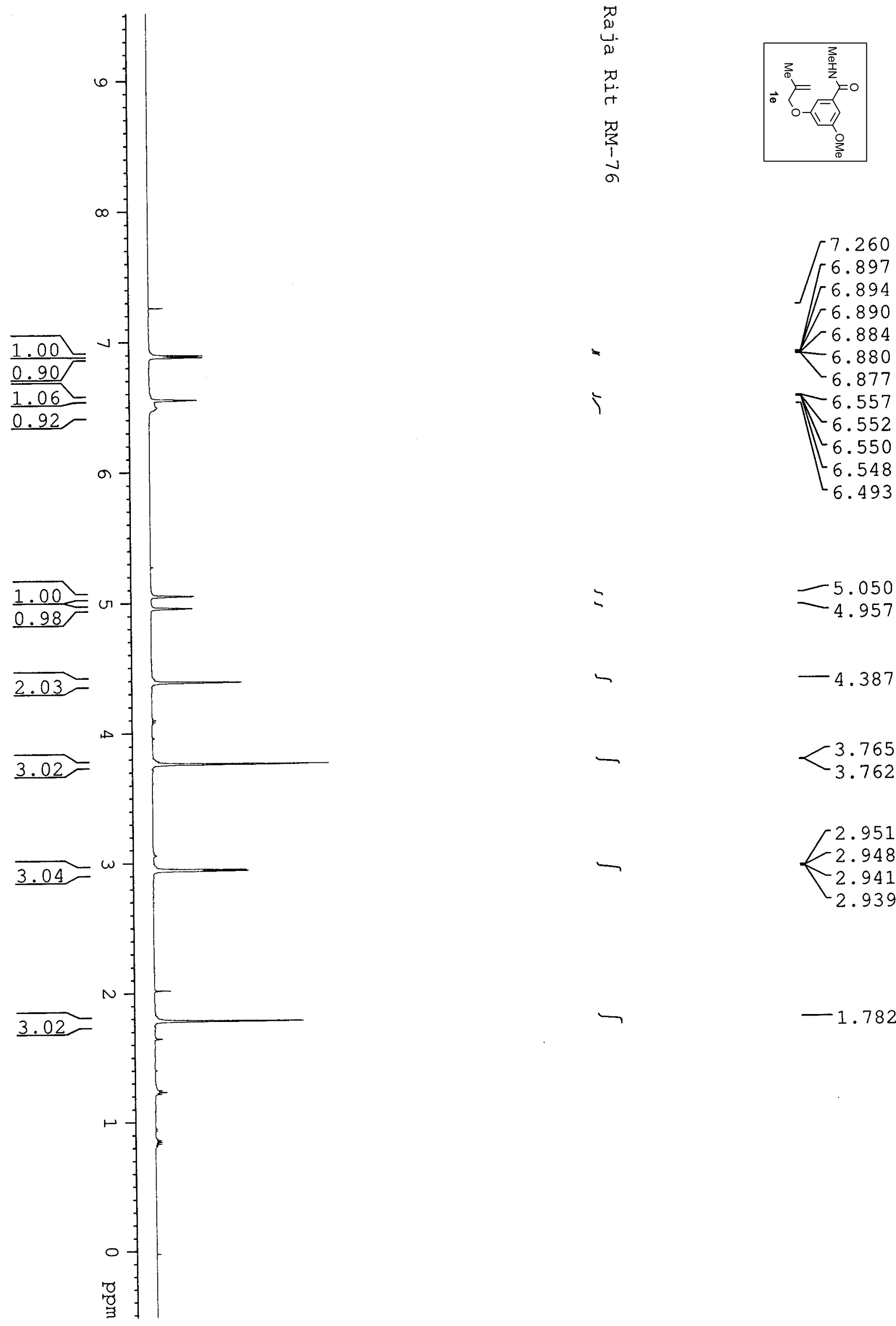

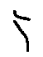

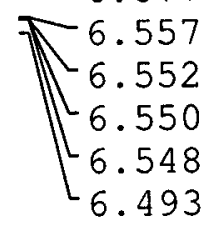

$=$

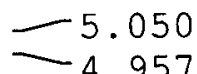

$-4.957$

ᄂ

$-4.387$

$-$

3.765

3.762

$\longrightarrow \quad \begin{aligned} & 2.951 \\ & 2.948 \\ & 2.941 \\ & 2.939\end{aligned}$

$-1.782$

윰

(. 182 


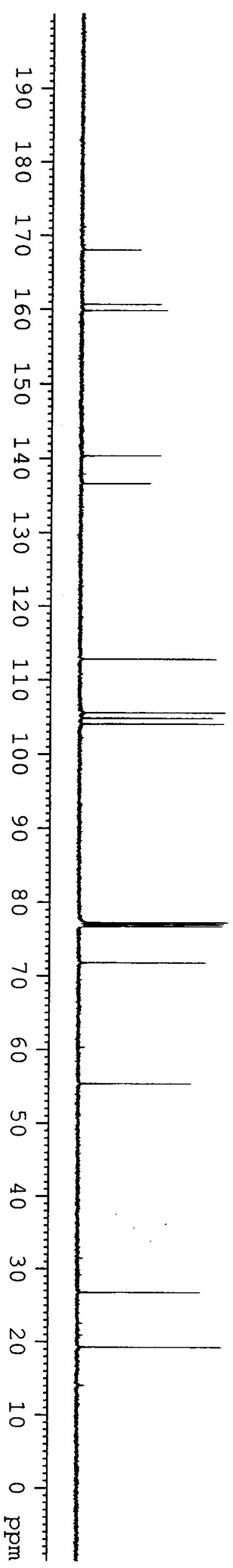

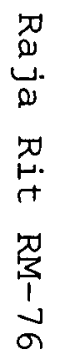

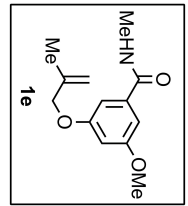

$-168.06$

$-160.68$

159.85

$-140.39$

$-136.66$

$-112.83$

105.58

104.83

104.08

77.26
77.00
76.75
71.79

$-55.41$

$-26.77$

$-19.29$ 

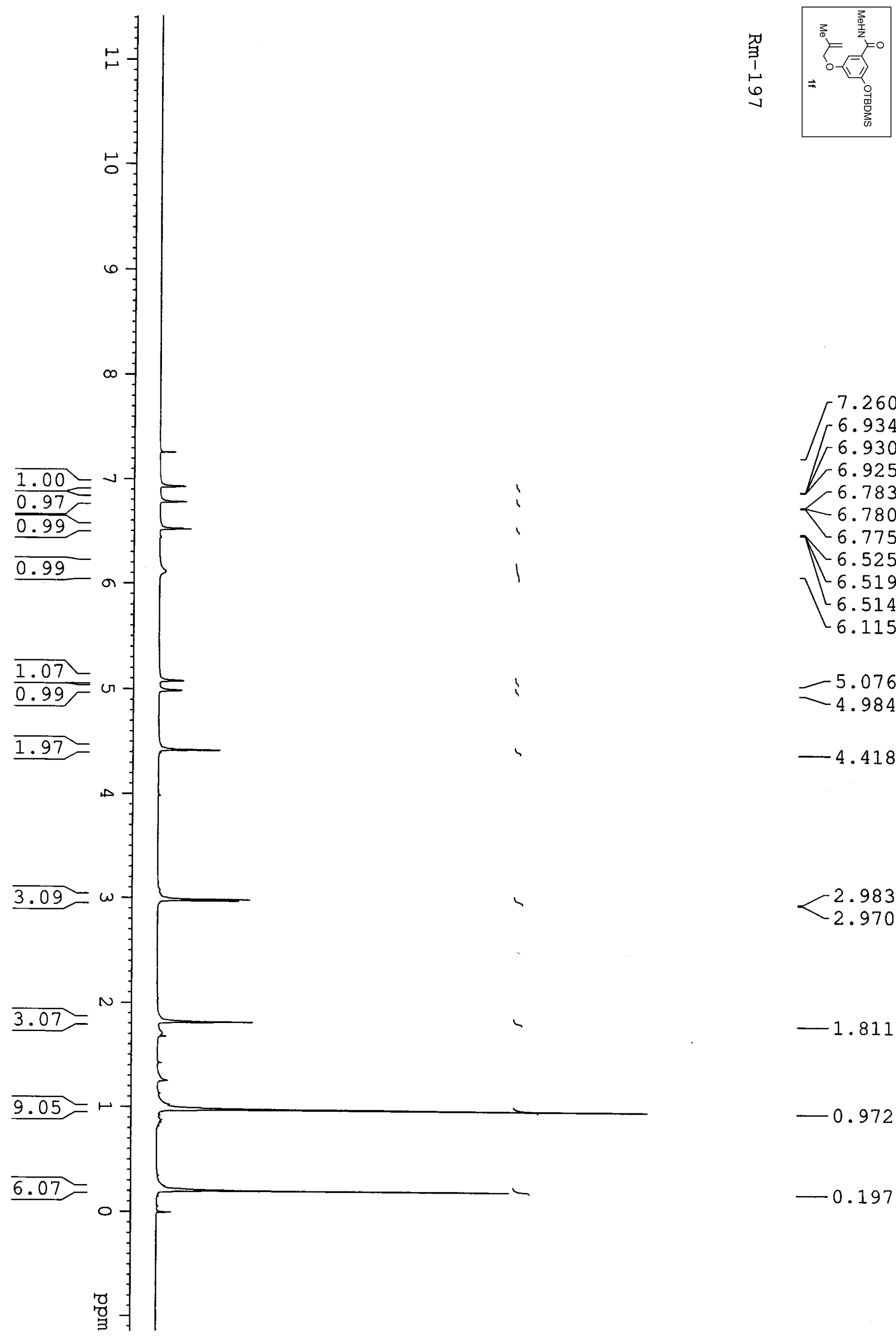

7.260

6.934

6.930

$-6.925$

6.783

$-6.780$

$-6.775$

$-6.525$

6.519

6.514

6.115

$-5.076$

$-4.984$

$-4.418$

2.983

2.970

$-1.811$

$-0.972$

$-0.197$ 

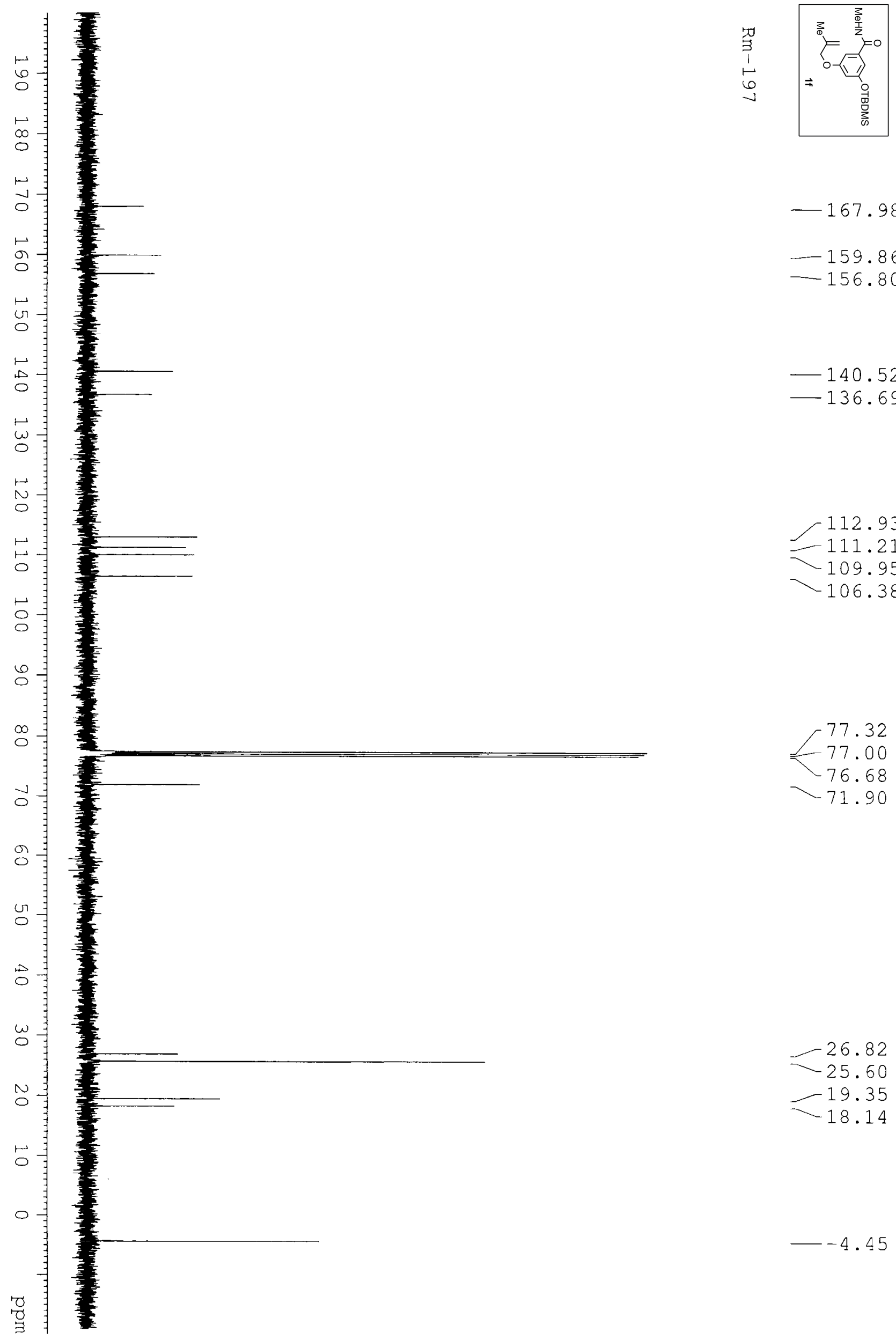

$-167.98$

$-159.86$

$-156.80$

$-140.52$

$-136.69$

$-112.93$

111.21
-109.95

$-109.95$

$-106.38$

77.32

$\lcm{-77.00}$

76.68

$-71.90$

$-26.82$

25.50

$-19.35$

$-18.14$

$--4.45$ 

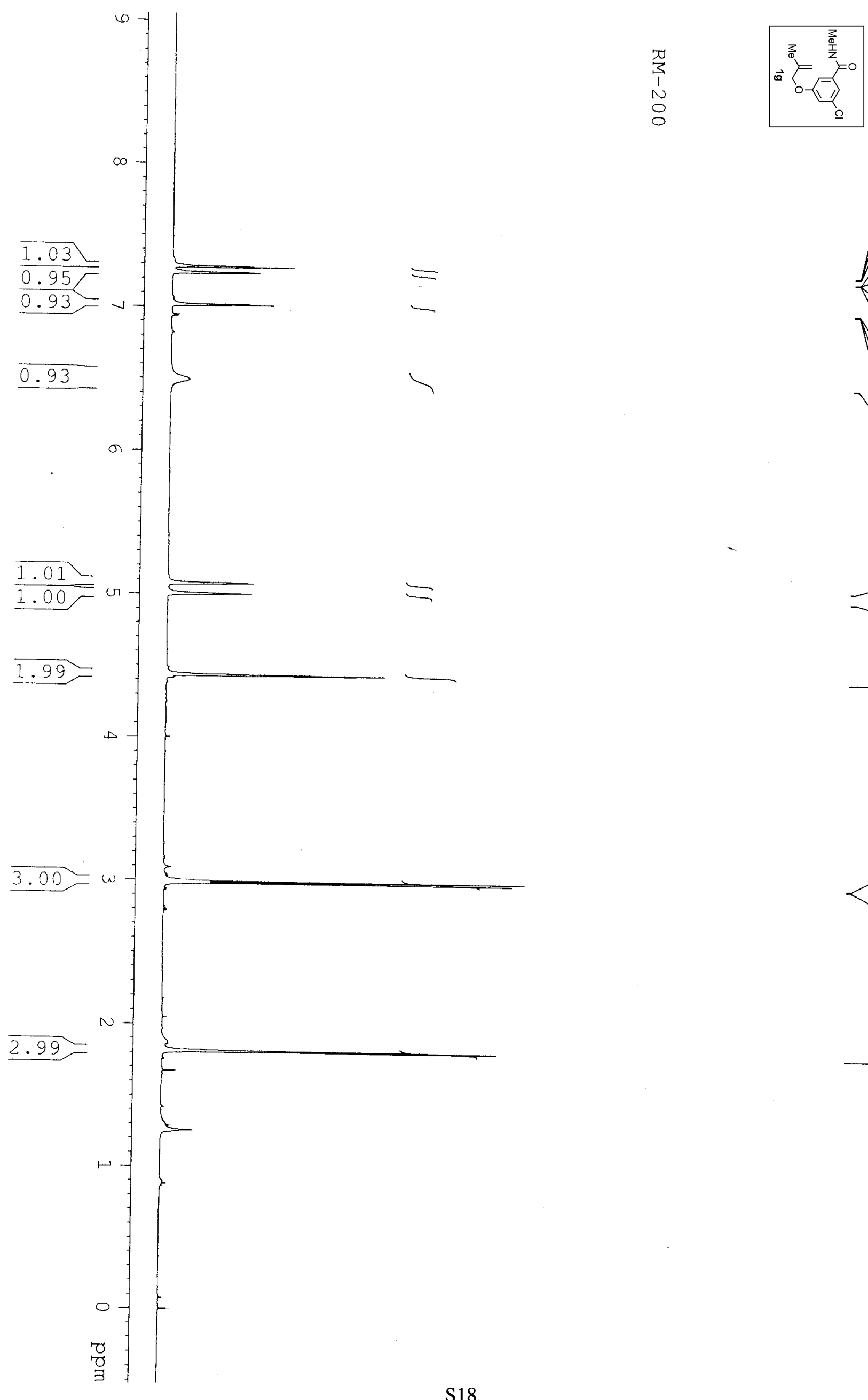

7.282

7.278

7.274

7.241

$-7.237$

$-7.235$

$-7.231$

$-7.016$

4.011

$\lfloor 7.005$

6.491

$-5.074$

$-5.000$

$-4.431$

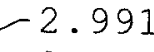

$<2.979$

$-1.808$ 


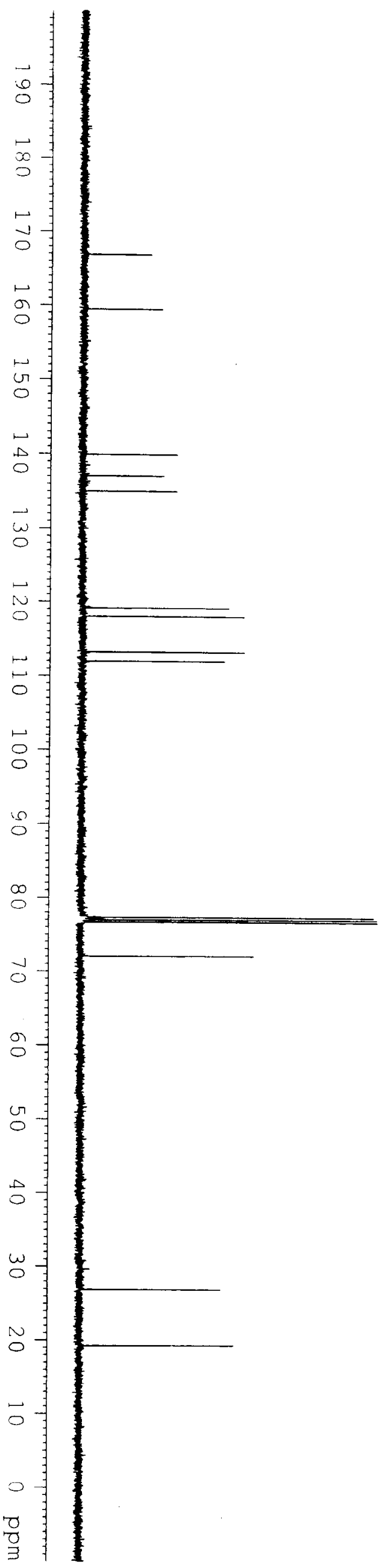

20
3
1
0
0
0

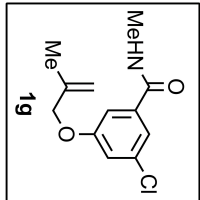

$-166.88$

$-159.46$

$-139.89$

$-137.06$

$-134.99$

$-119.14$

118.03

$-113.22$

$-111.95$

77.32

77.00

76.68

$-72.03$

$-26.89$

$-19.27$ 

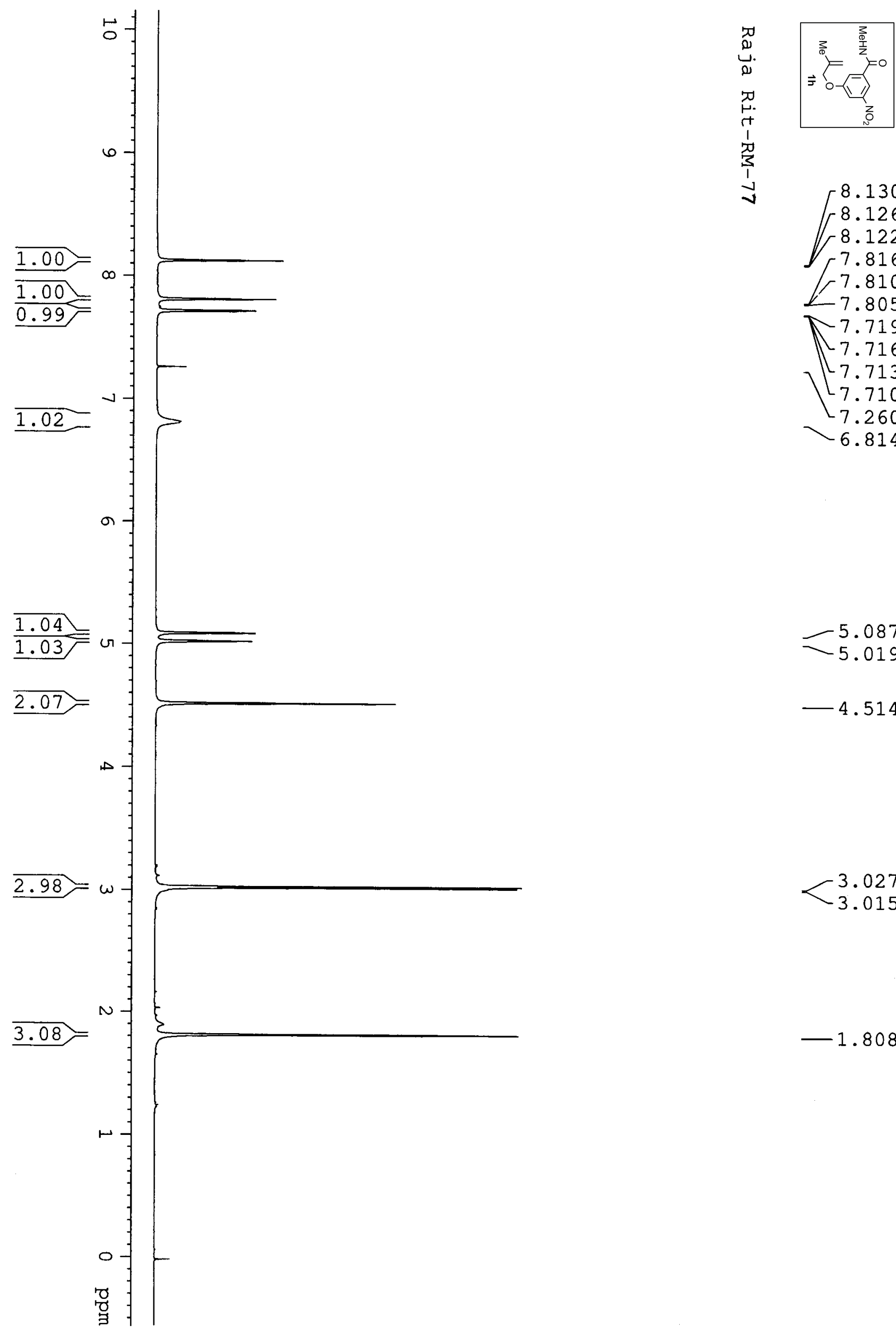

8.130
8.126

8.122

7.816

7.810

$-7.805$

$-7.719$

7.716

$-7.713$

7.710

7.260

6.814

$-5.087$

$-5.019$

$-4.514$

$<3.027$

3.015

$-1.808$ 

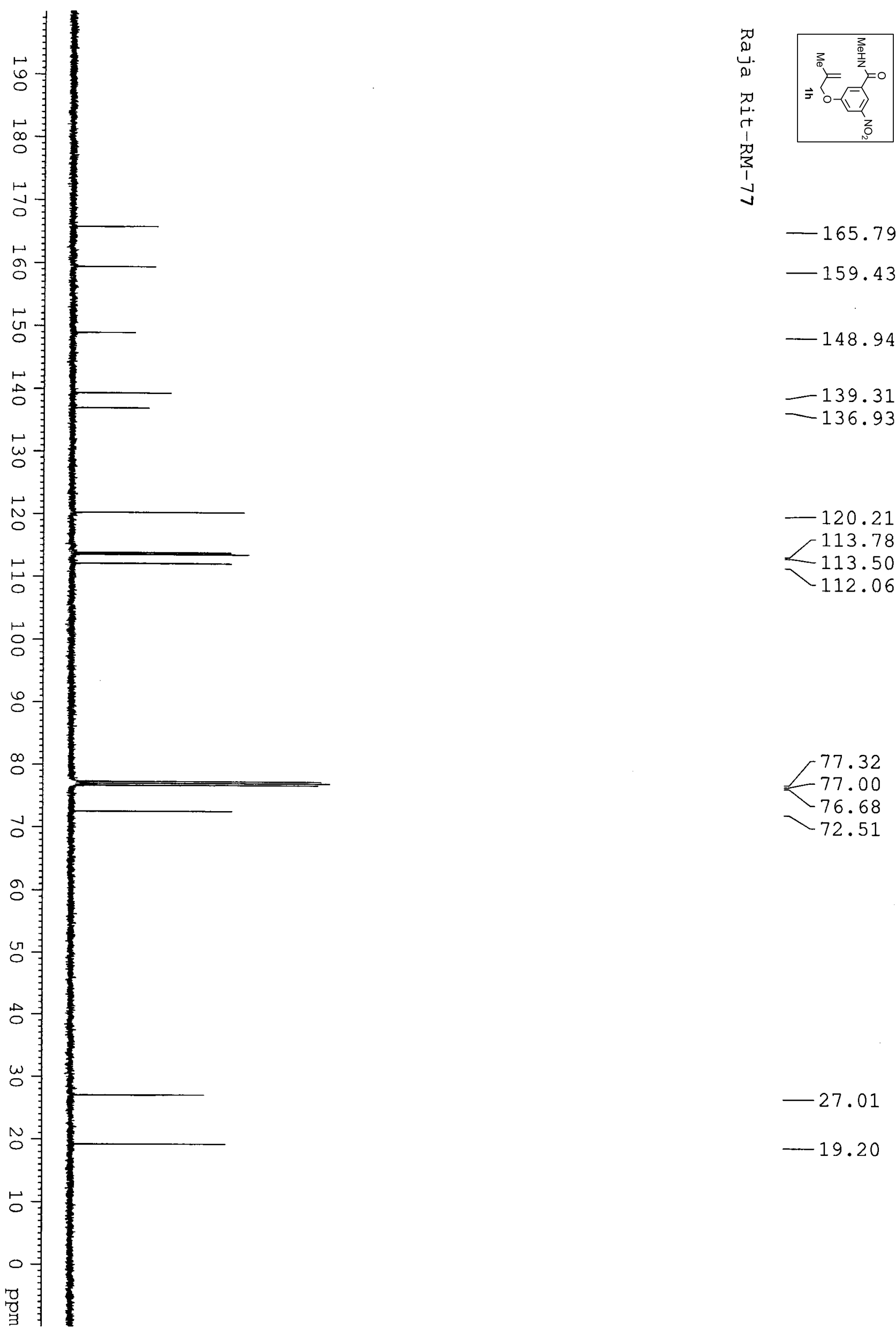

$-165.79$

$-159.43$

$-148.94$

$-139.31$

$-136.93$

$-120.21$

113.78

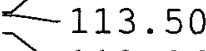

112.06

77.32

$\{77.00$

$-76.68$

72.51

$-27.01$

$-19.20$ 


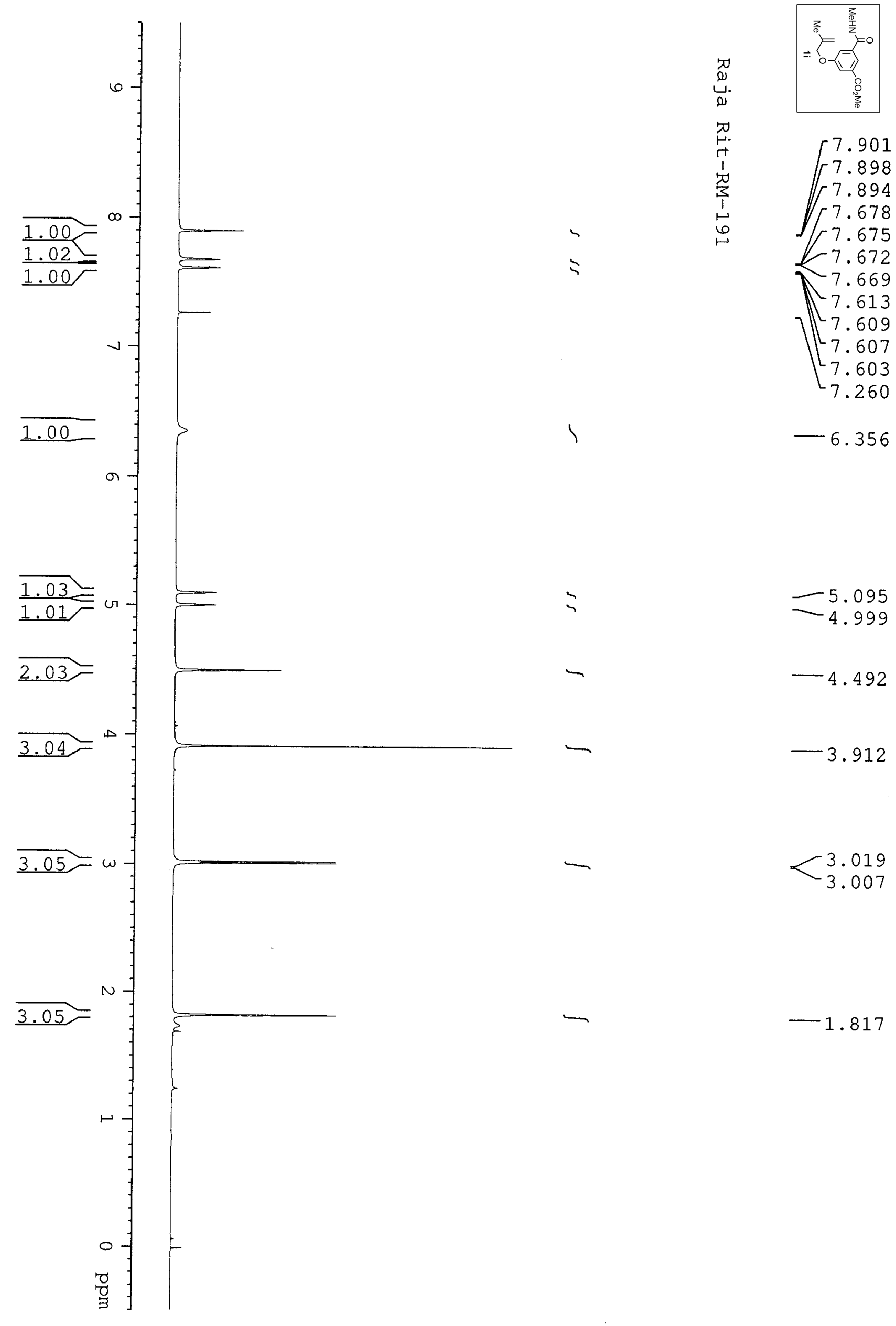



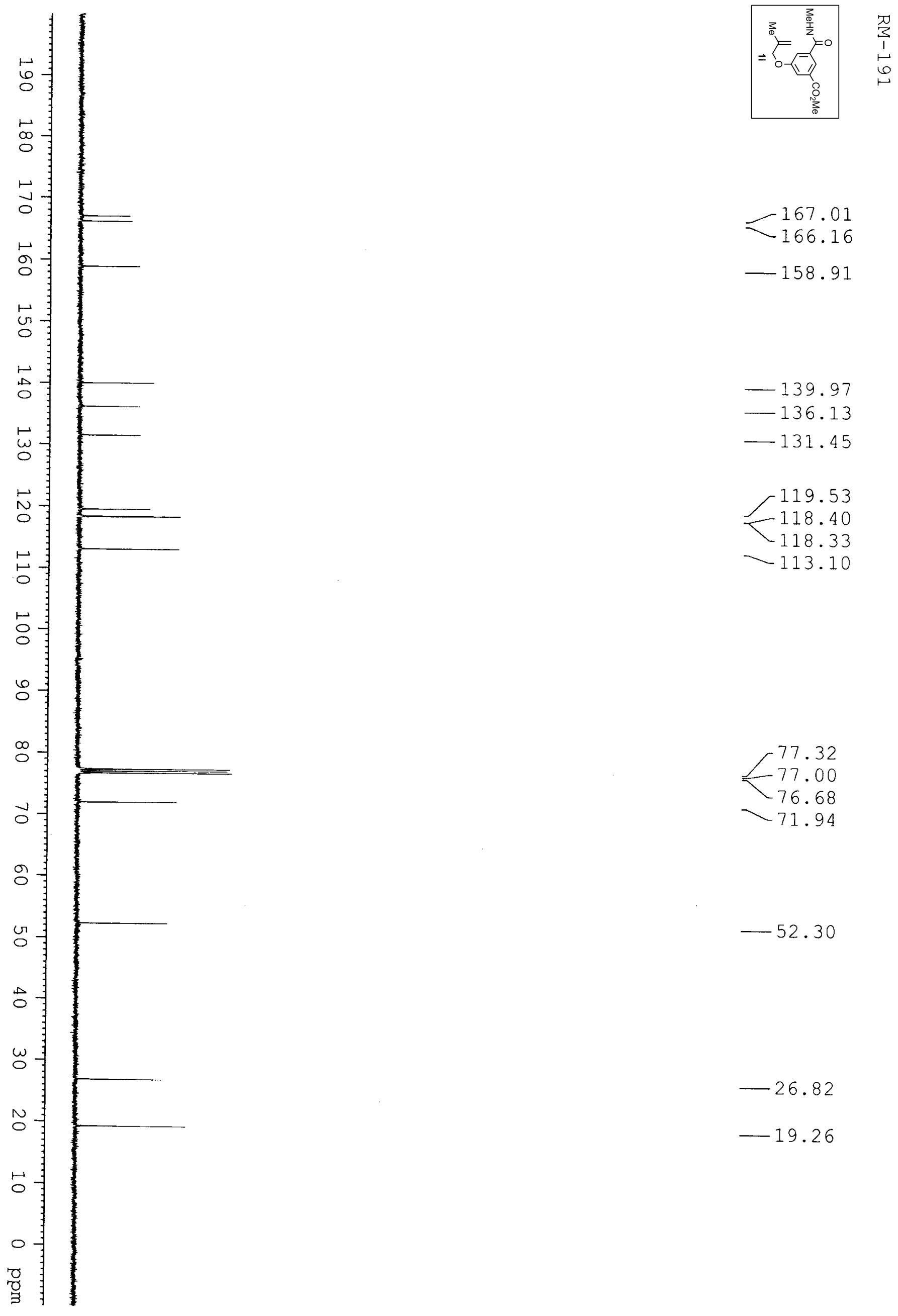

$-136.13$

$-131.45$

$-119.53$

118.40

118.33

$-113.10$

77.32
$<77.00$
76.68
71.94

$-52.30$

$-26.82$

$-19.26$ 


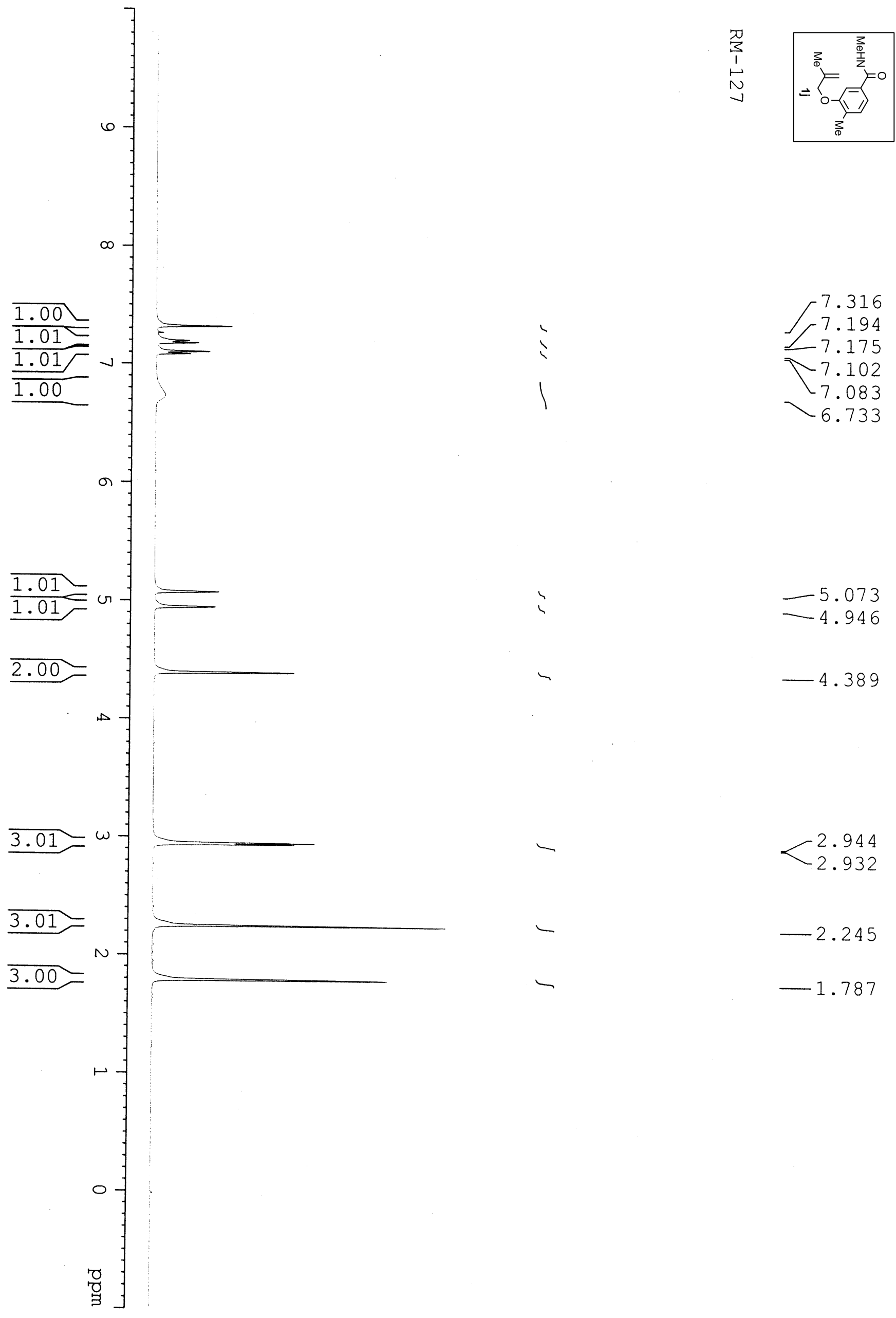




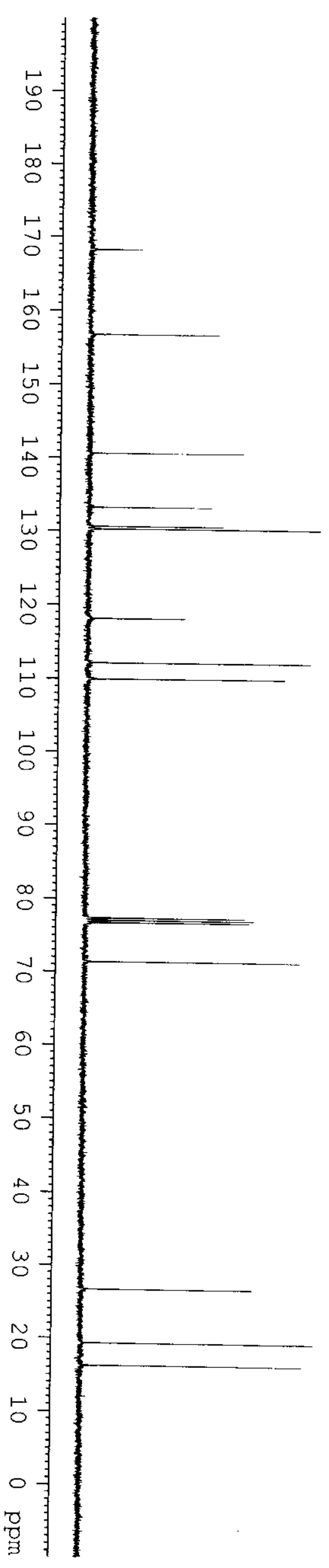

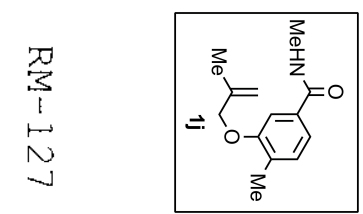

$-168.21$

$--156.76$

$-140.61$

$-133.21$

130.59

130.24

$-118.16$

$-112.15$

$-109.96$

77.32

77.00

$-76.68$

$-71.33$

$-26.68$

$-19.28$

$-16.21$ 

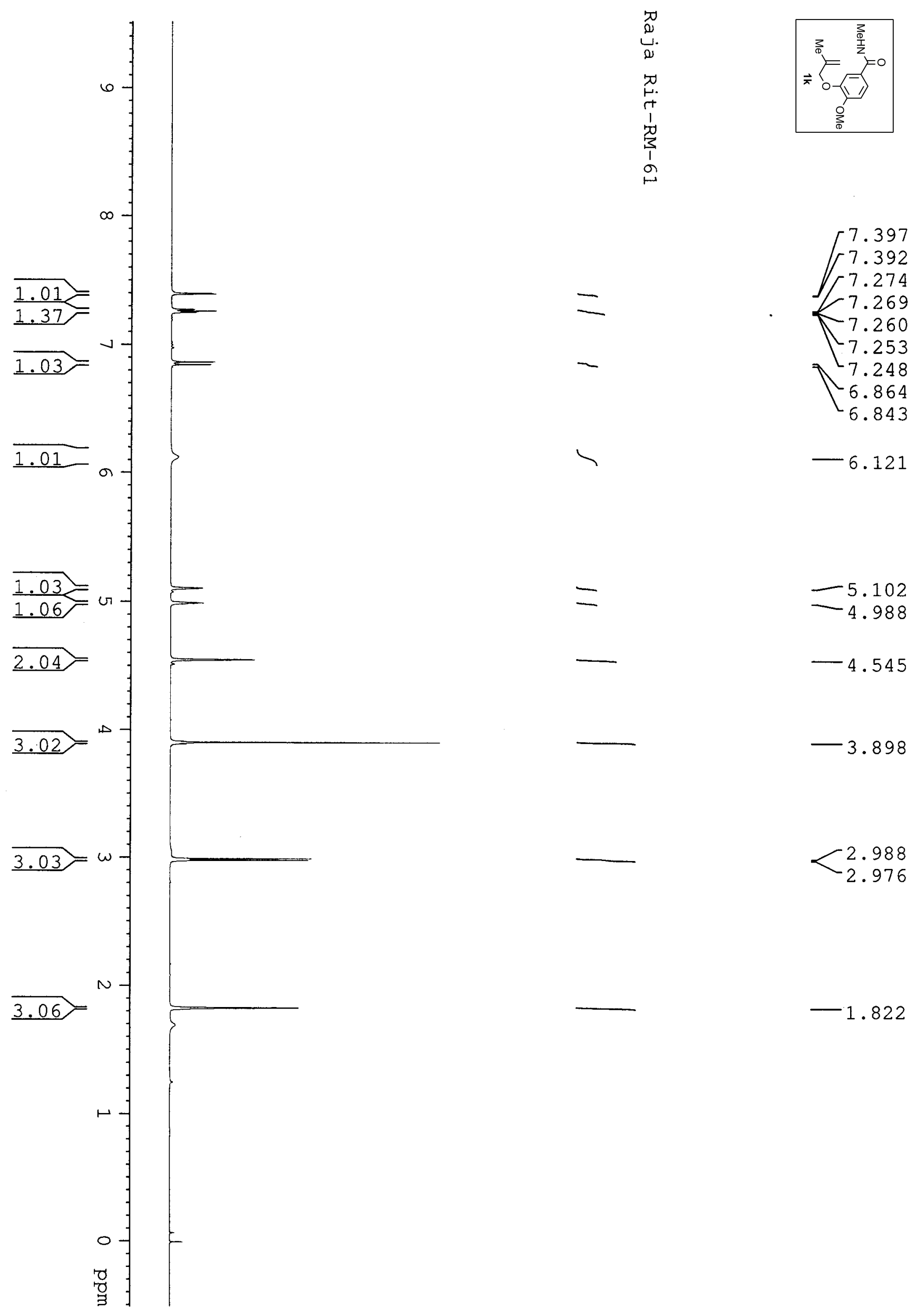

$-4.545$

$-3.898$

$-2.988$

$-2.976$

$-1.822$ 

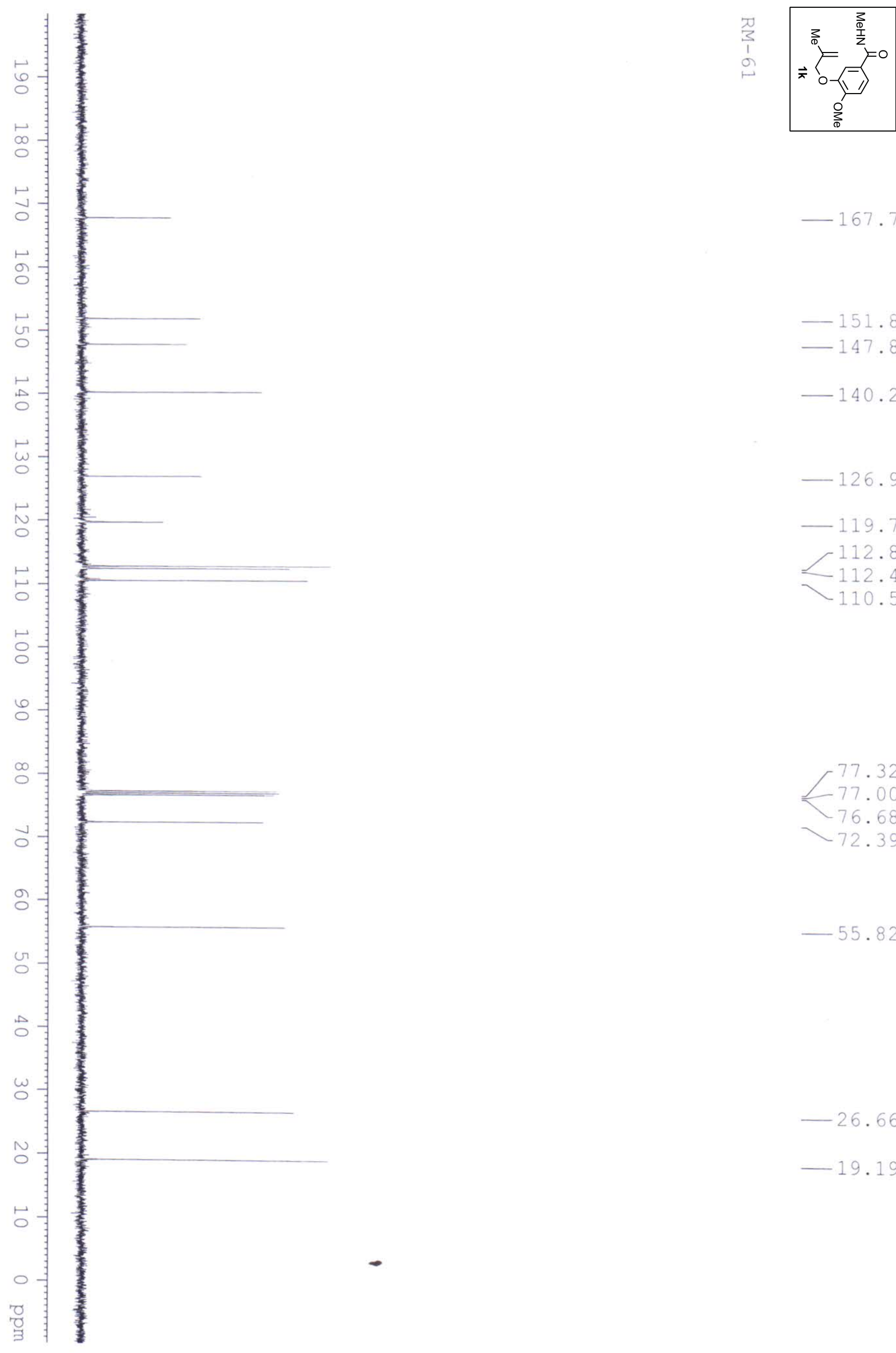

$-167.77$

$-151.88$ 147.82 40.20

$-126.94$

$-119.70$

$-112.82$

$-112.45$

110.57

77.32

$-77.00$

76.68

$-72.39$

$-55.82$

$-26.66$

$-19.19$ 

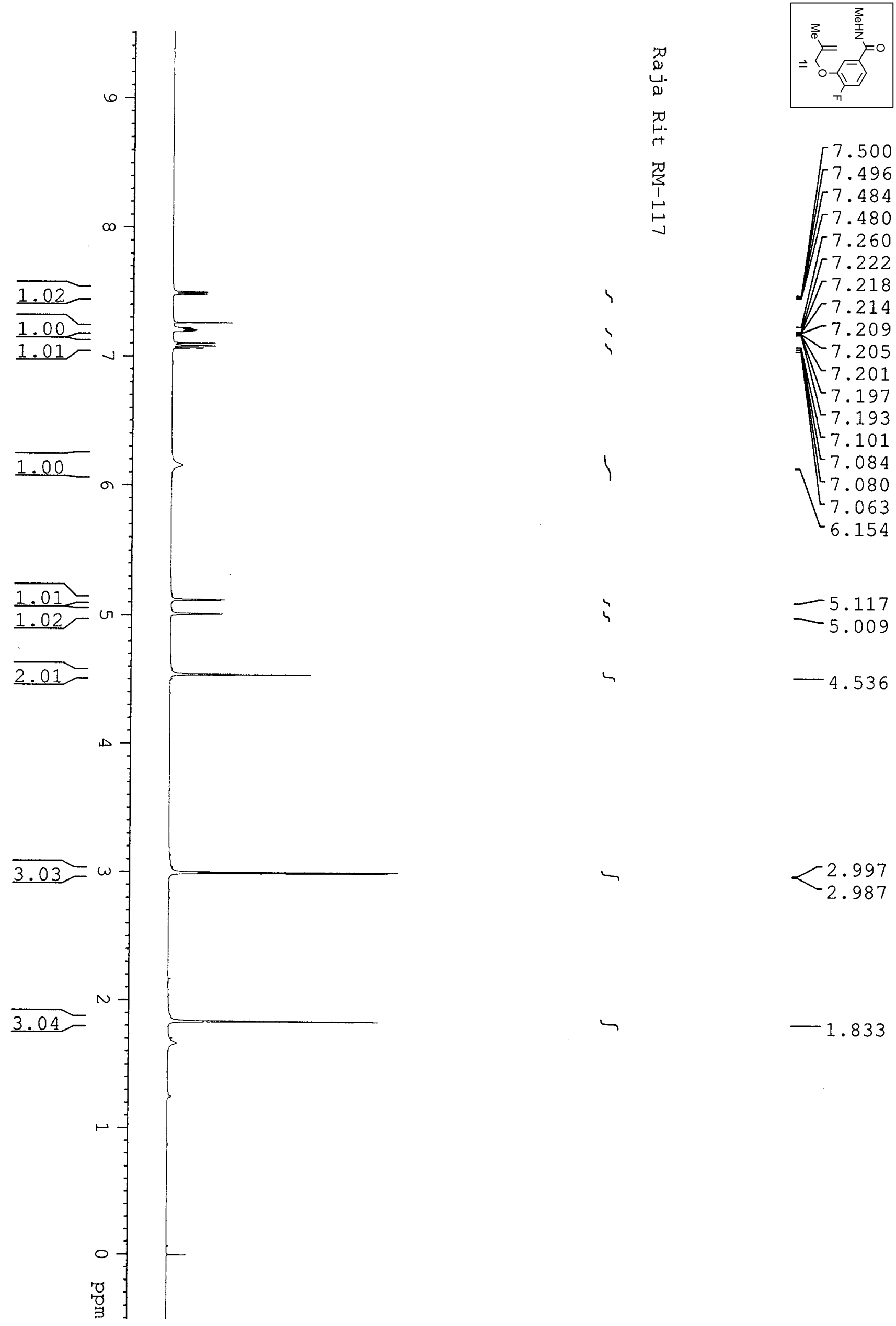

思

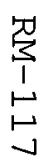

7.500

7.496

7.484

7.480

7.260

7.222

7.218

7.214

$-7.209$

$-7.205$

$-7.201$

7.197

7.193

7.101

7.084

7.080

7.063

6.154

-5.117
-5.009

$-4.536$

4

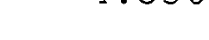

4

2.997
2.987

$-1.833$

$\checkmark$

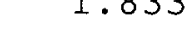



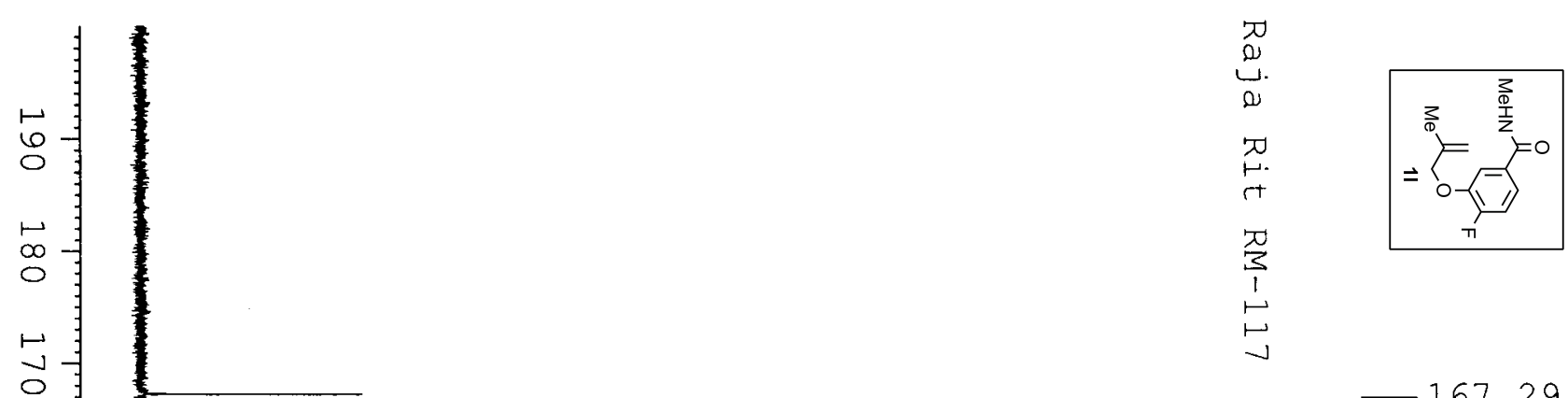

$-167.29$

$-155.51$

$-153.51$

$+146.76$

$<146.68$

$-139.87$

$-130.95$

$-130.93$

$\begin{array}{r}119.43 \\ \hline\end{array}$

115.90

$-115.74$

114.58

113.31

๖용

○

$\infty$

旁

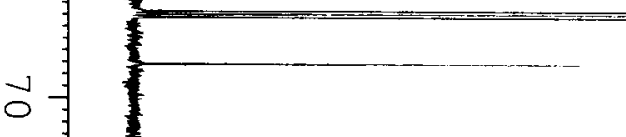

77.26
-77.00
776.75
72.84

u

$\stackrel{\circ}{\circ}$

w

N

$-19.12$

เ

묵 

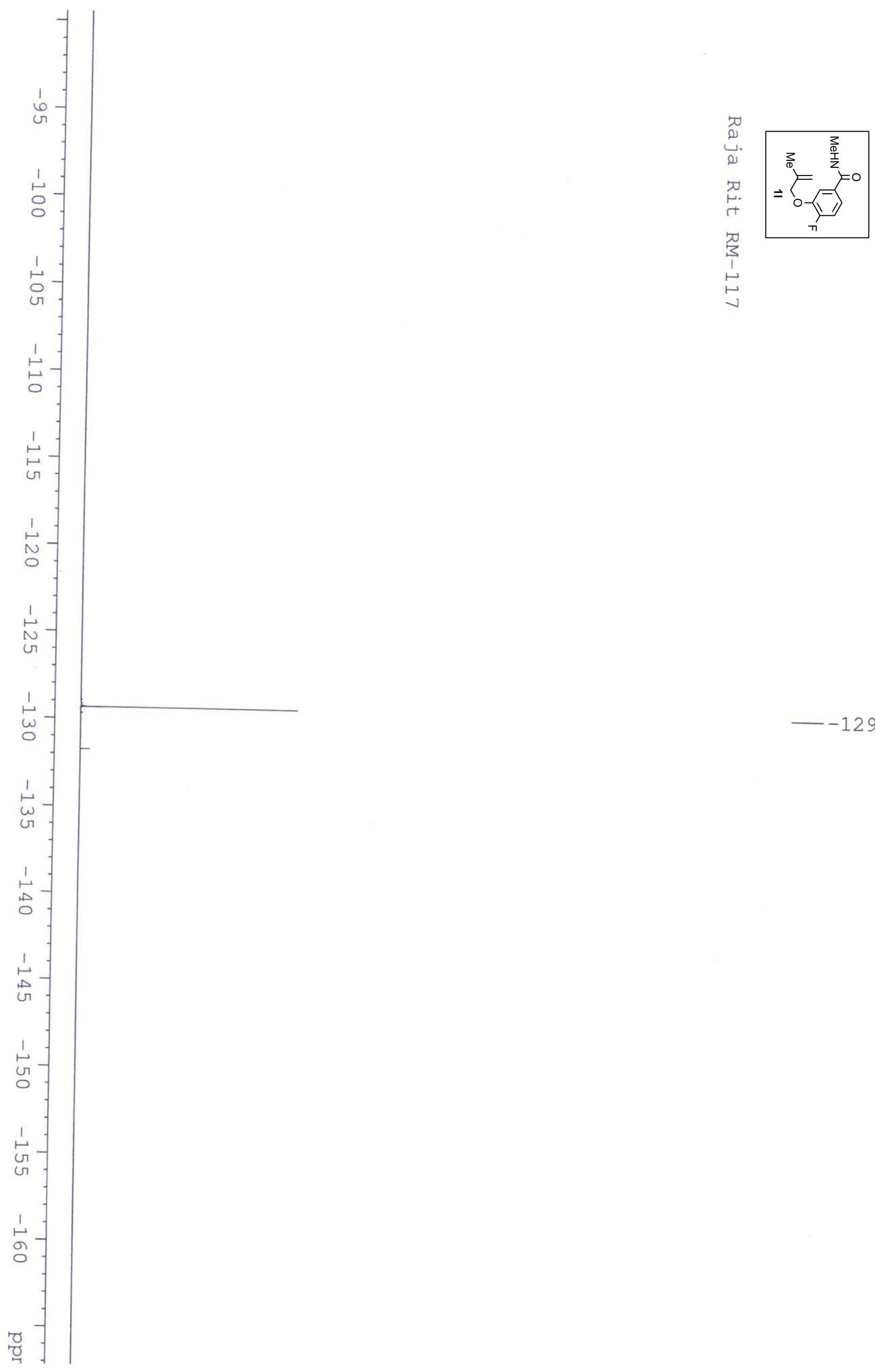

$--129.35$ 

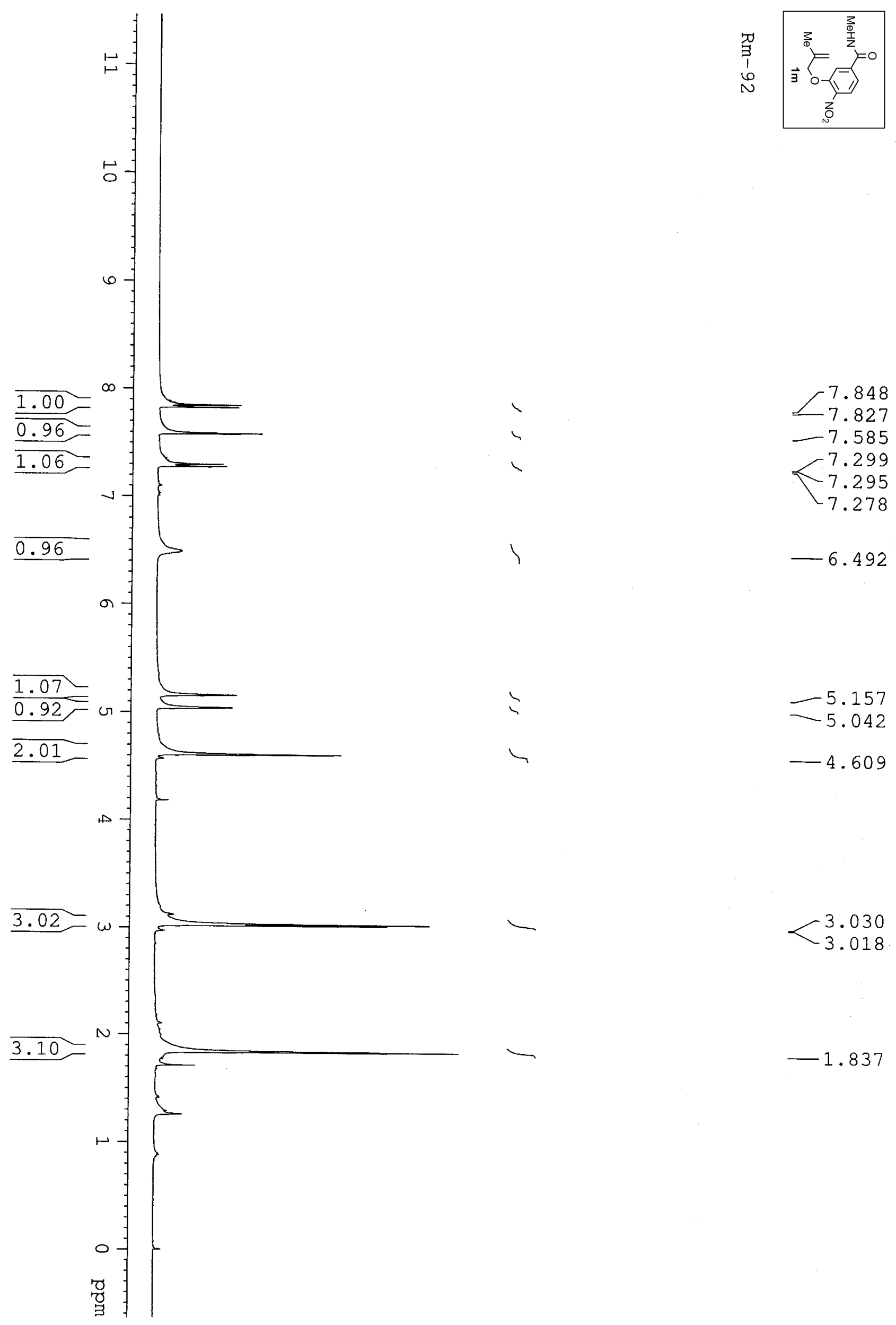

$-6.492$

$-5.157$

$-5.042$

$-4.609$

$-3.030$

3.018

$-1.837$ 


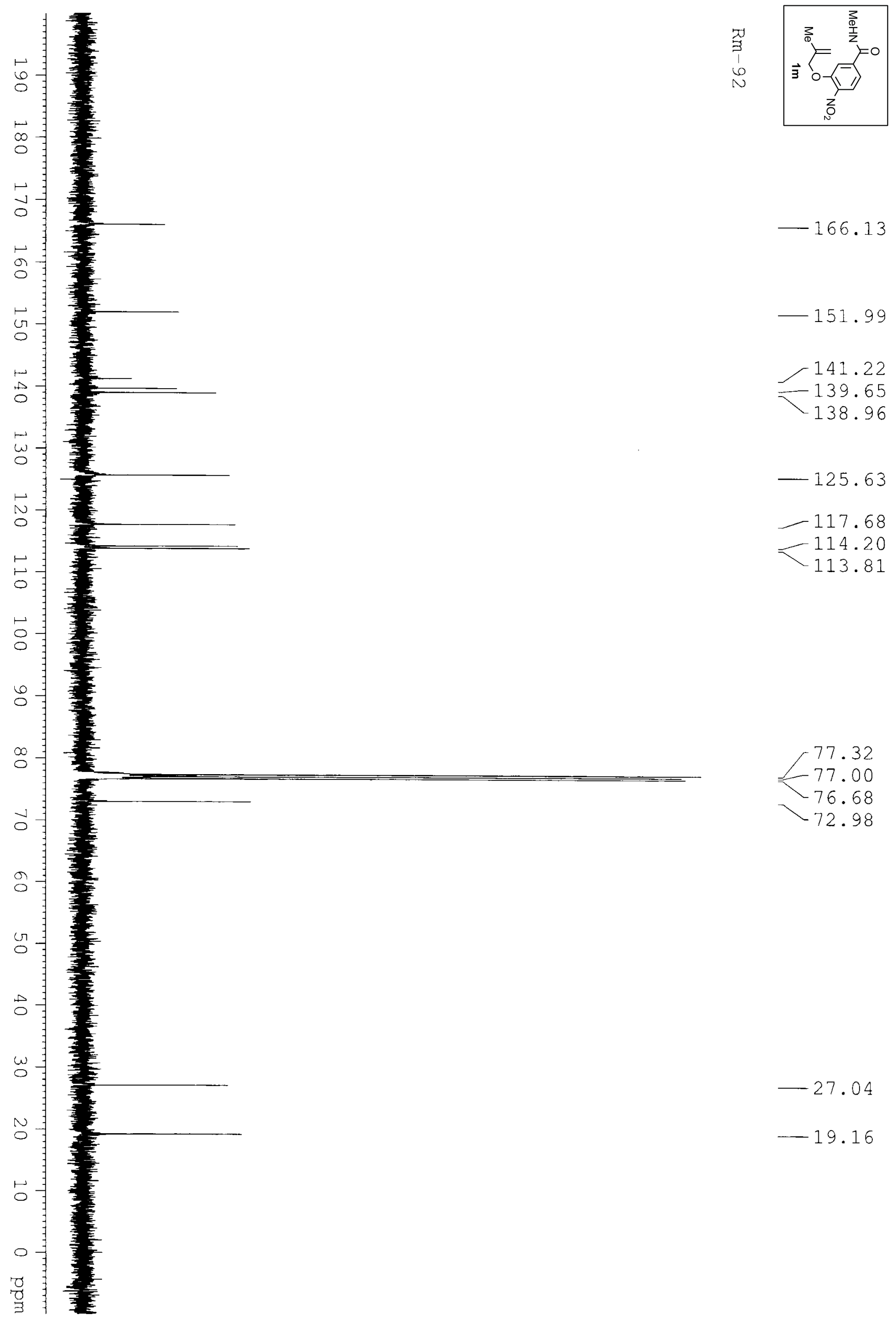



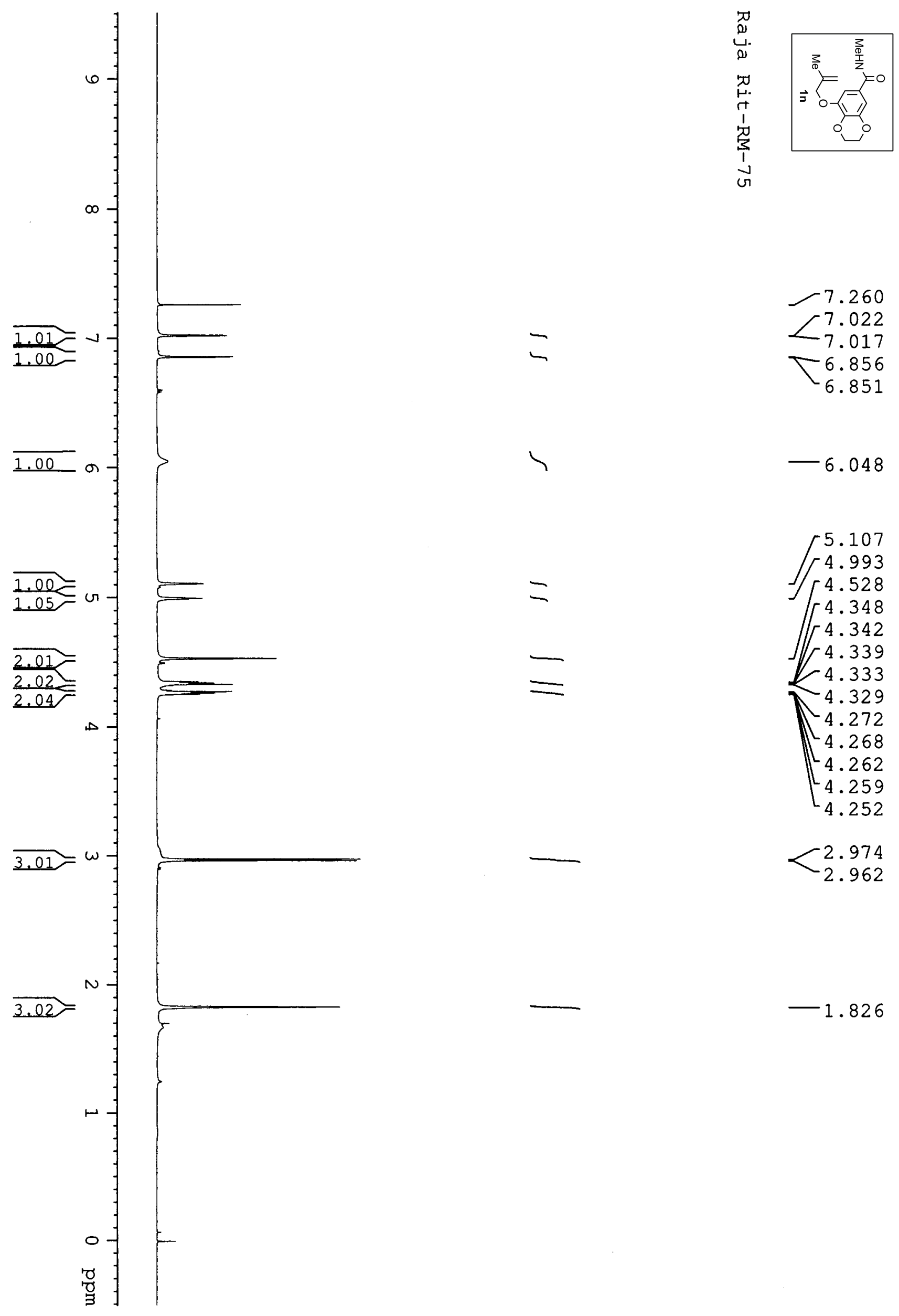

$=$

$\int \begin{aligned} & 5.107 \\ & 4.993 \\ & {\left[\begin{array}{l}4.528 \\ 4.348\end{array}\right.}\end{aligned}$

4.348

4.342

4.339

$=$

4.333

$-4.329$

4.272

4.268

4.262

4.259

4.252

-

$<2.974$

2.962

$-1.826$

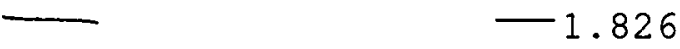



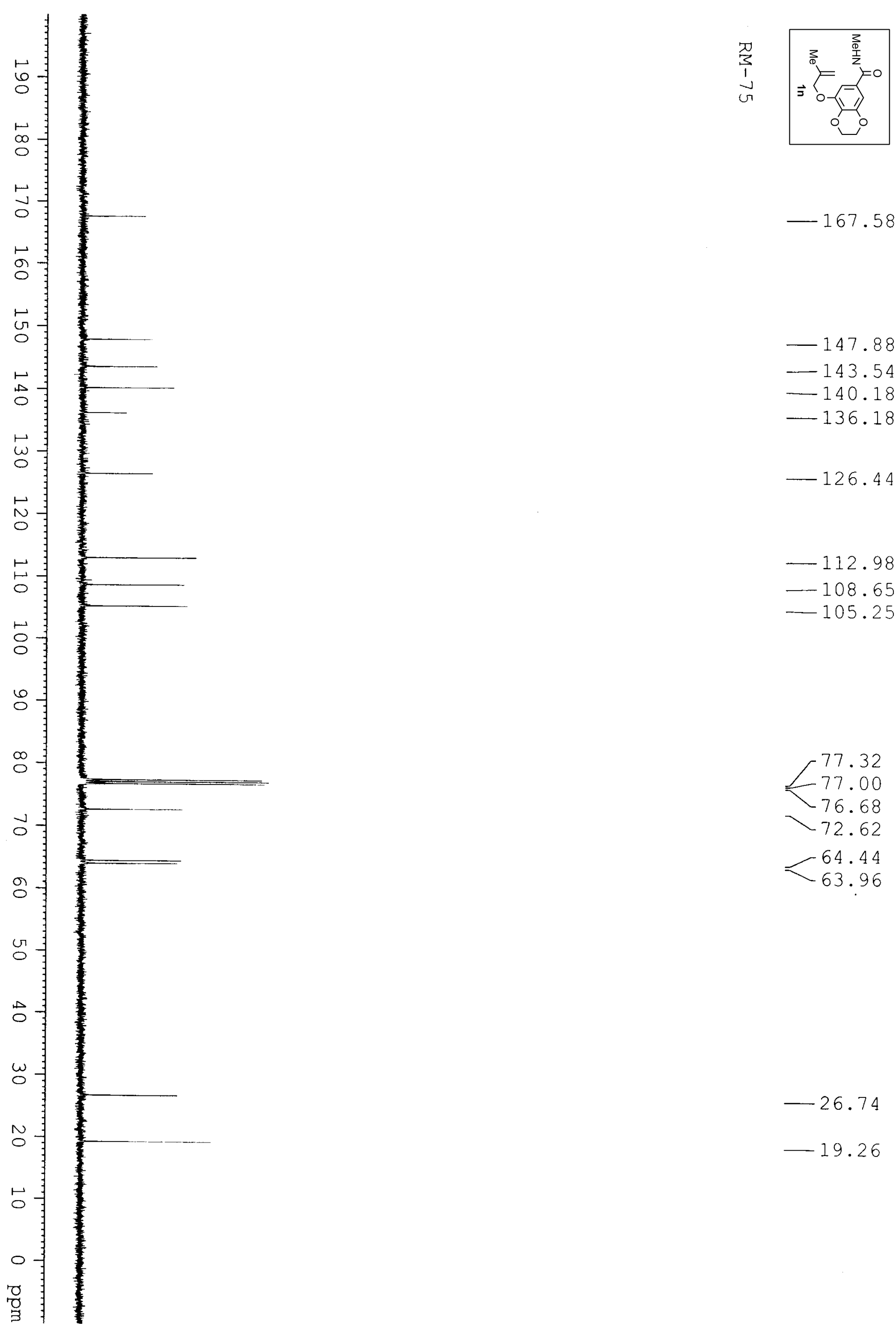

$-147.88$

$-143.54$

$-140.18$

$-136.18$

$-126.44$

$-112.98$

$-108.65$

$-105.25$

77.32

$\lcm{-77.00}$

$-76.68$

$-72.62$

64.44
-63.96

$-26.74$

$-19.26$ 

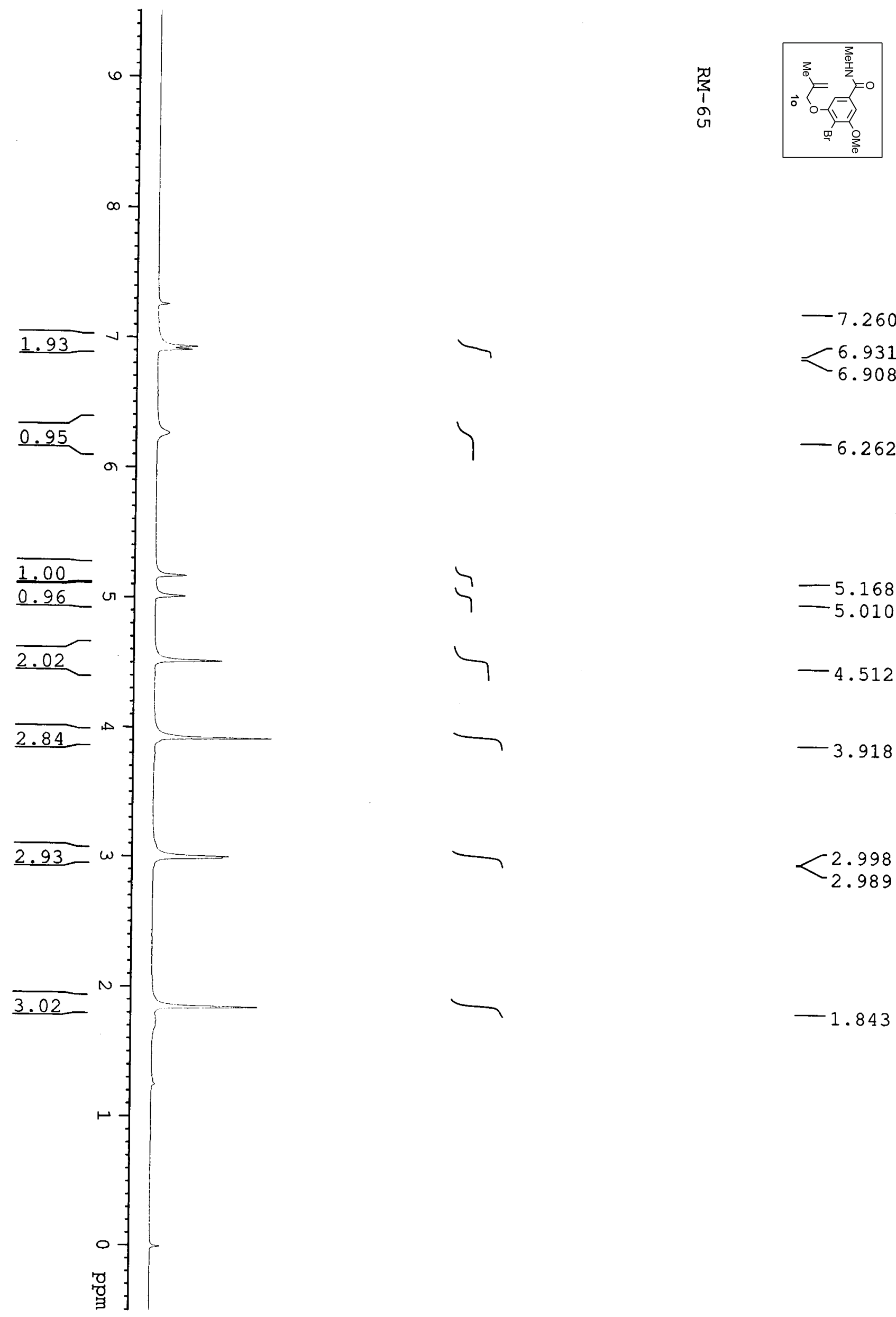

6.931

6.908

$-6.262$

$-5.168$

$-5.010$

$-4.512$

$-3.918$
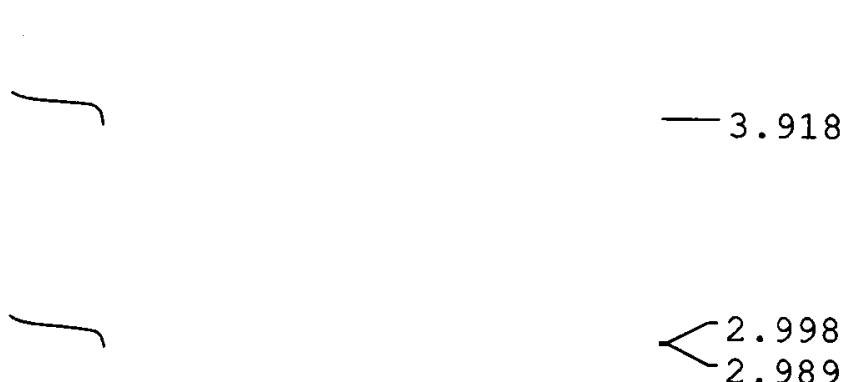

$\checkmark$

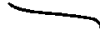

2.989

$-1.843$ 


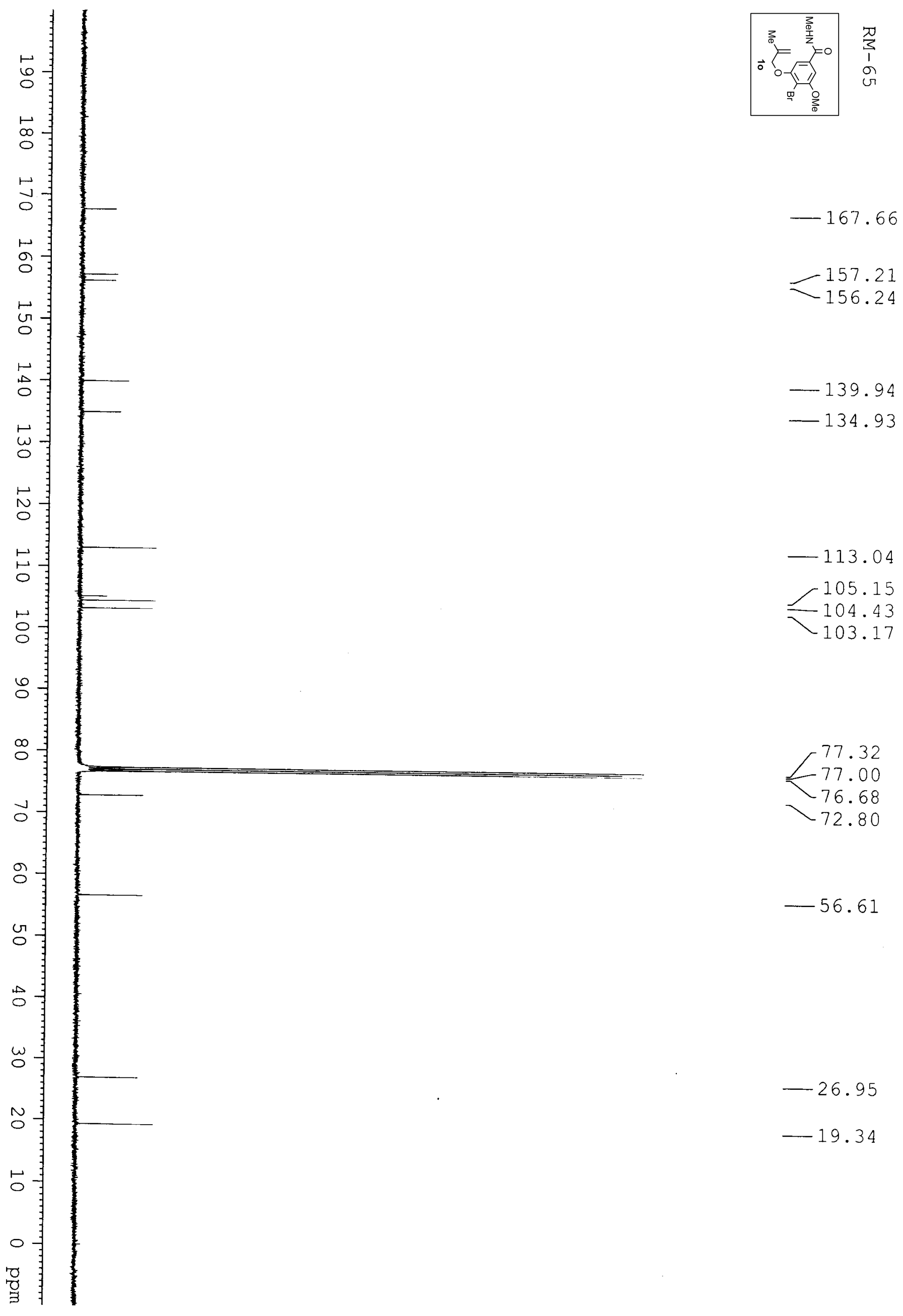




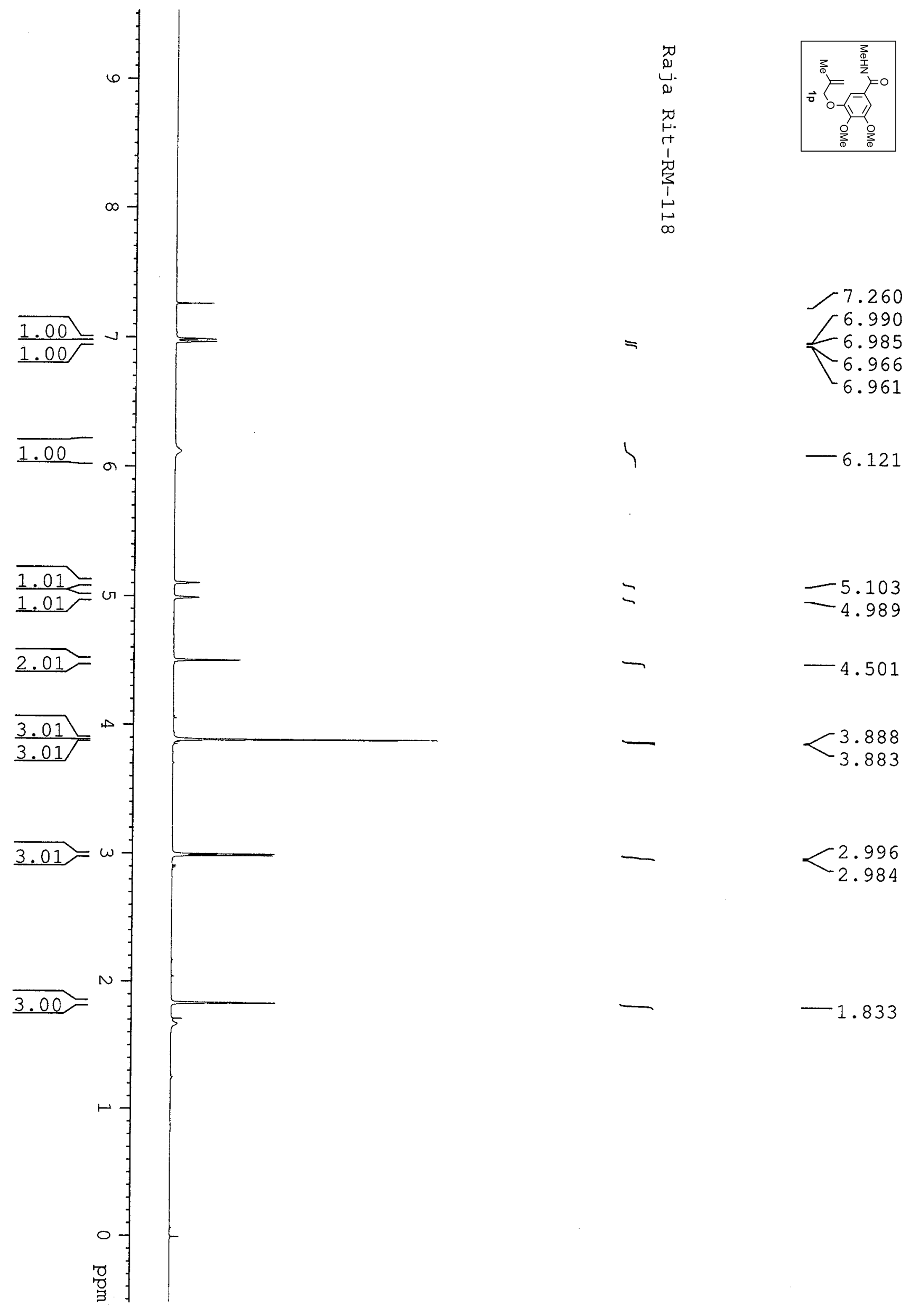




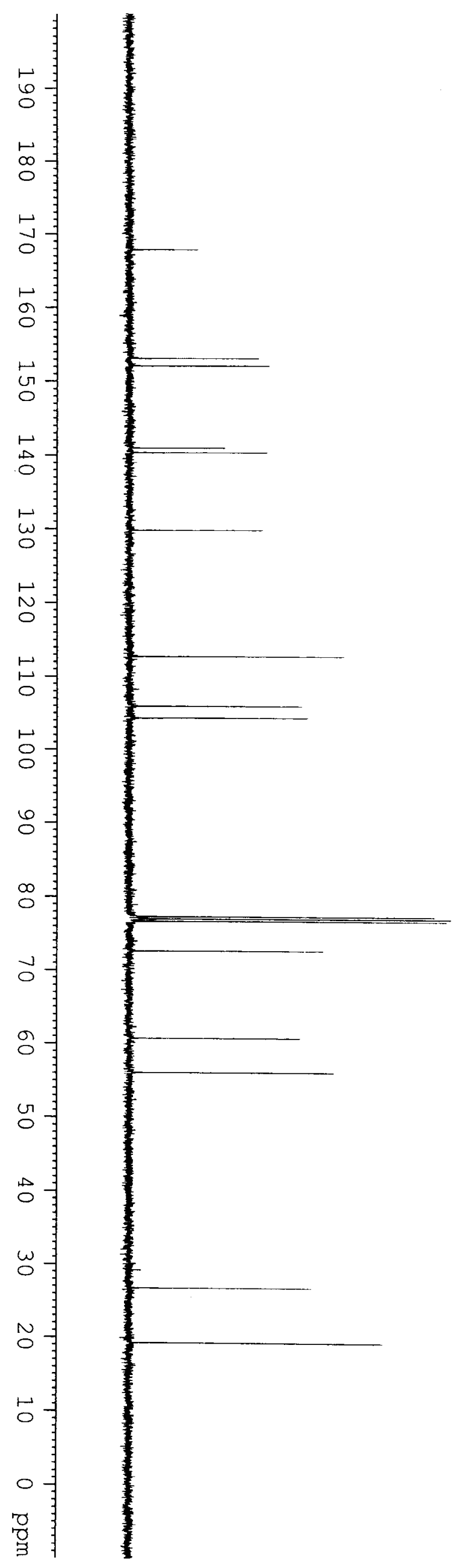

$=0$

$-167.88$

$+153.12$

$-152.11$

140.98
-140.34

$-140.34$

$-129.81$

$-112.73$

$-105.91$

$-104.30$

77.32

$\lcm{-77.00}$

76.68

$-72.64$

$-60.74$

$-56.07$

$-26.78$

$-19.27$ 


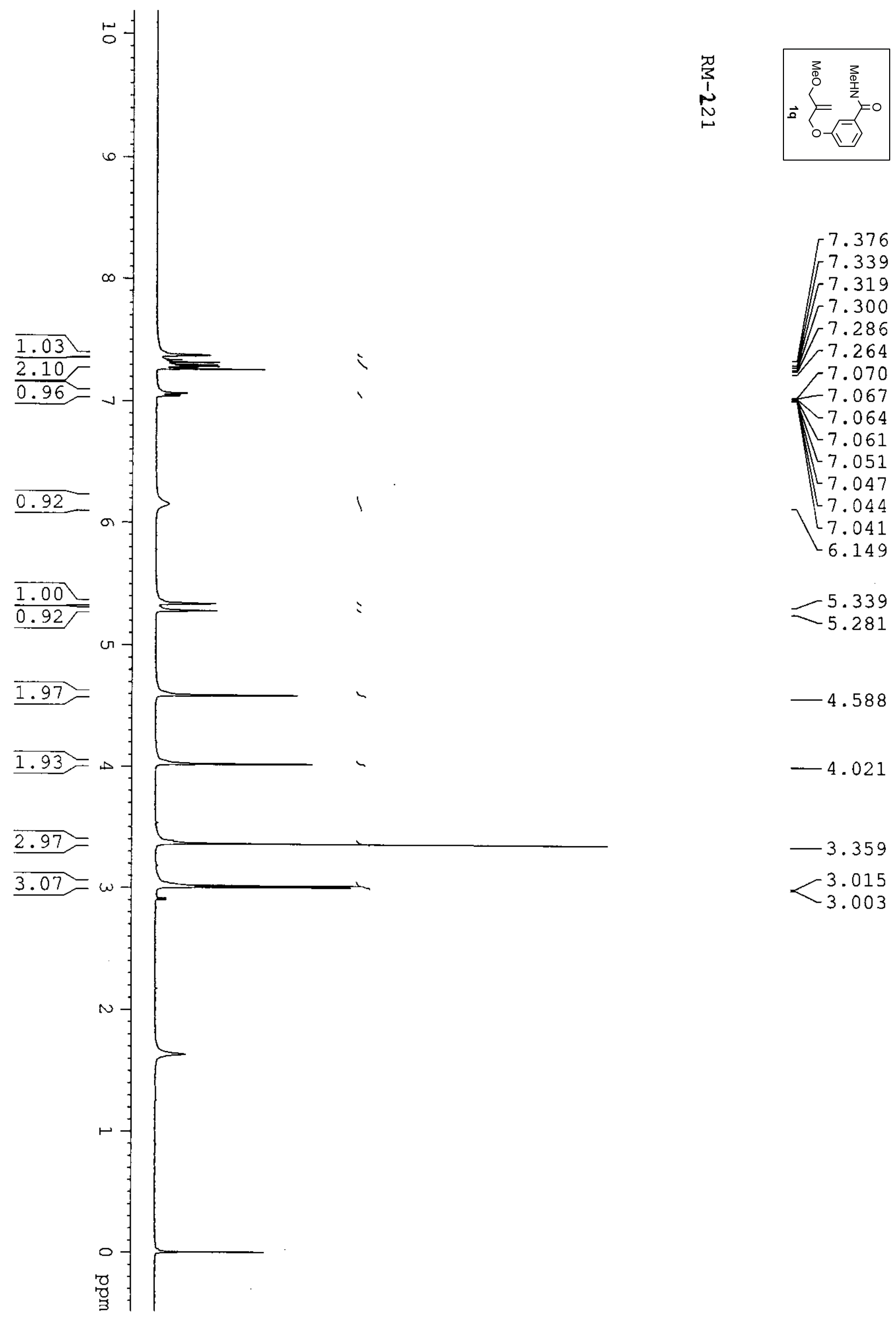



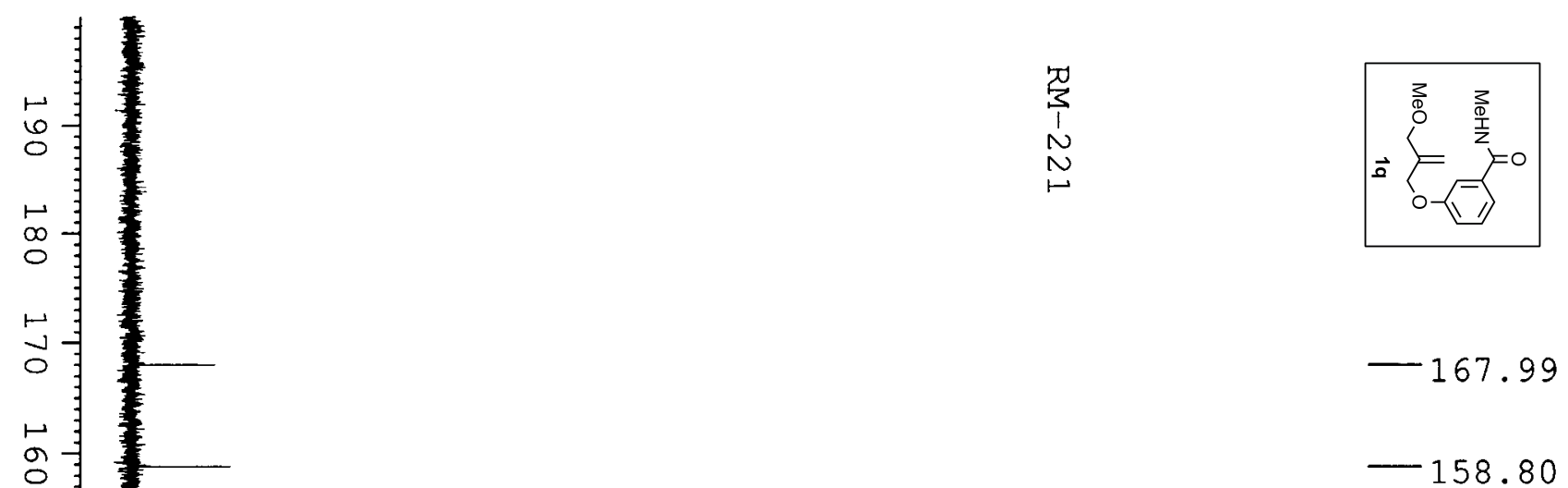

$-141.00$

$-136.04$

$-129.52$

118.87

$-118.10$

$-115.10$

113.19

占

๖

๖

$\infty$

$\infty$

$\checkmark$

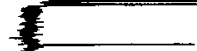

的事

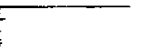

$\pi$

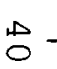

w

영

$-26.82$

77.32

$<77.00$

76.68

$-73.26$

$-68.51$

$-58.10$

要

$\circ$

읔 


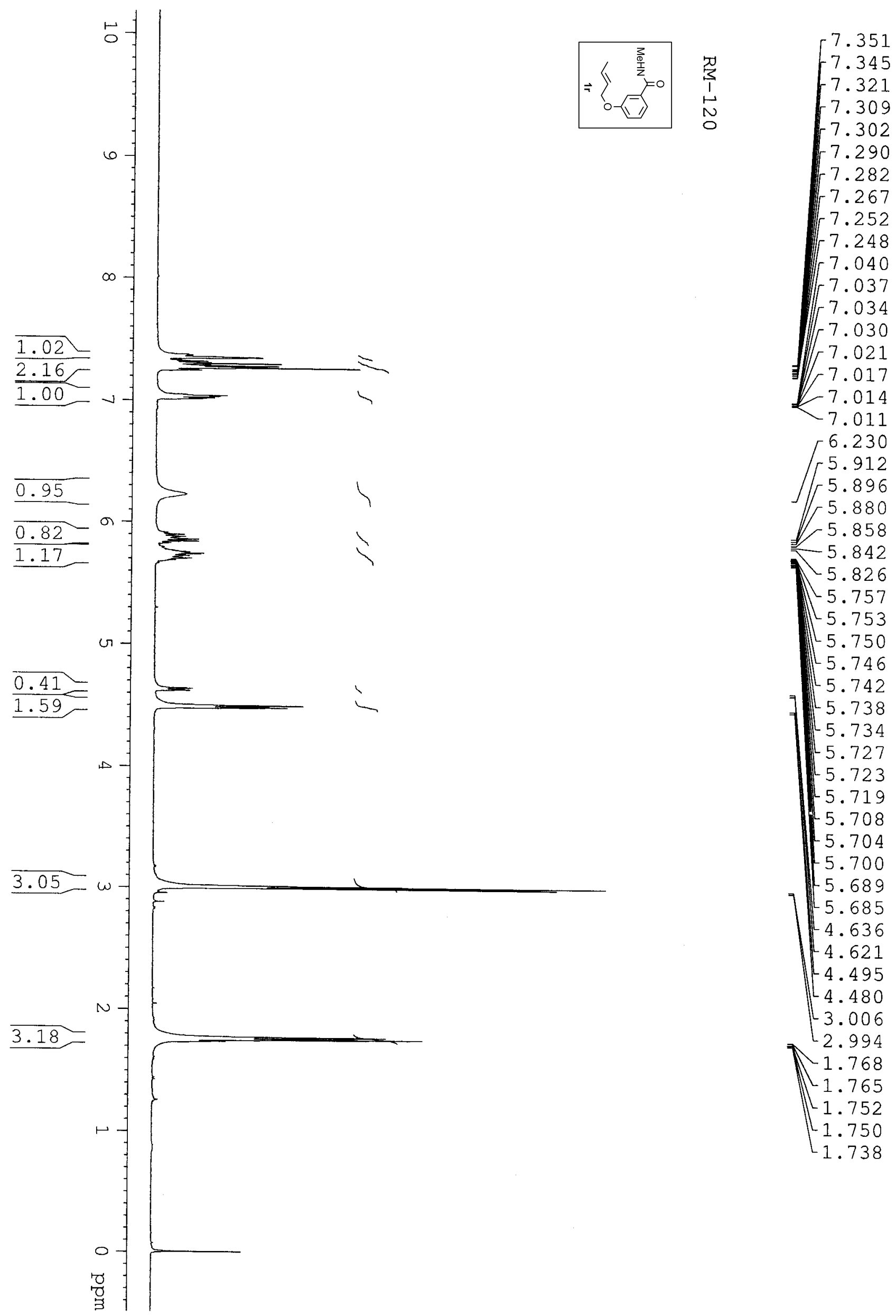



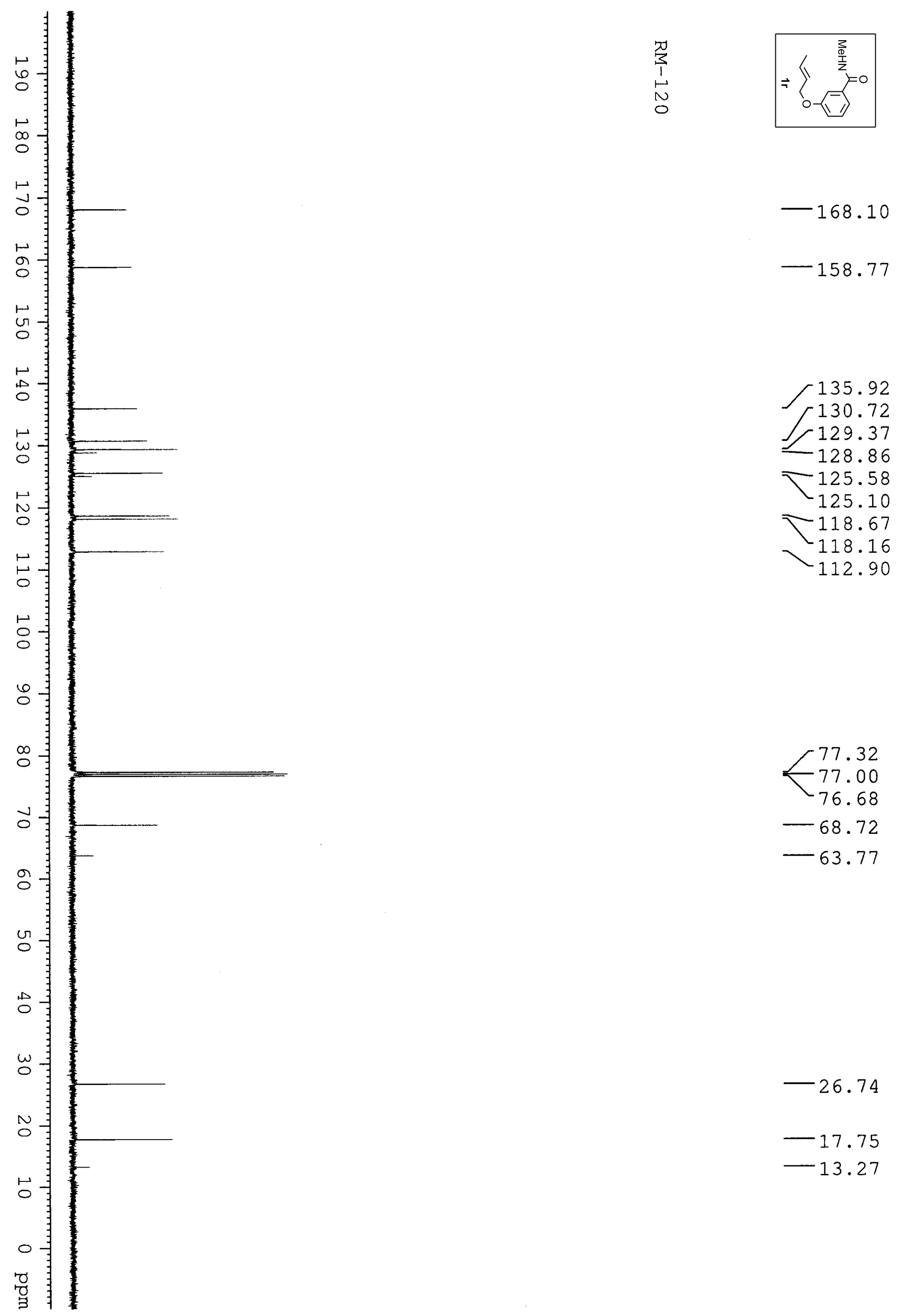

135.92

130.72

129.37

$-128.86$

$\checkmark 125.58$

125.10

$-118.67$

118.16

112.90

77.32

$-77.00$

76.68

$-68.72$

$-63.77$

$-26.74$

$-17.75$

$-13.27$ 

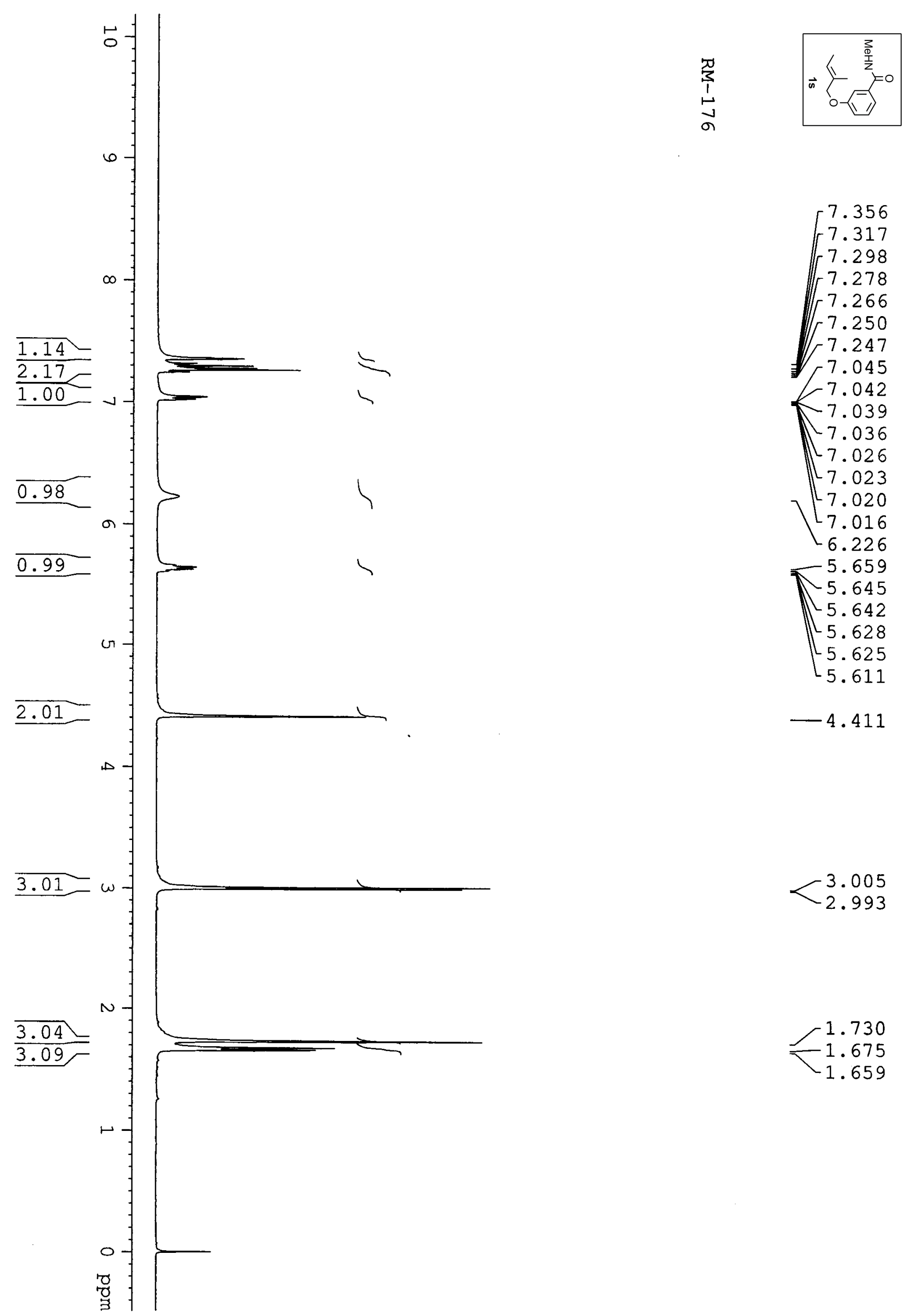

7.356

7.317

7.298

7.278

7.266

7.250

7.247

7.045

$-7.042$

$-7.039$

$-7.036$

7.026

7.023

7.020

$-7.016$

$-6.226$

5.659

$-5.645$

5.642

5.628

5.625

5.611

$-4.411$

$<3.005$

2.993

1.730

$-1.675$

1.659 

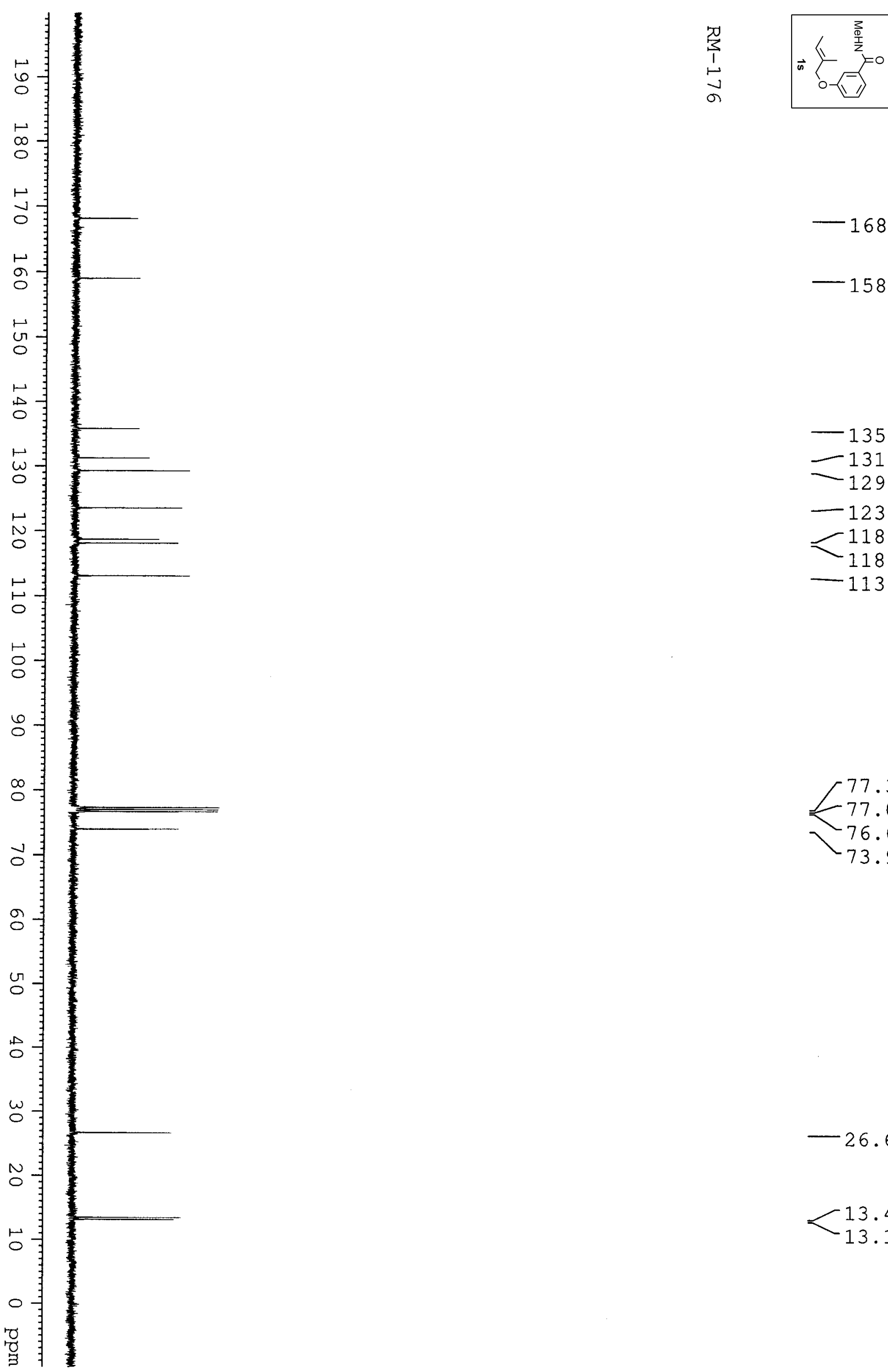

$-168.14$

$-158.99$

$-135.82$

$-131.24$

$-129.27$

$-123.51$

$-118.66$

$-118.09$

$-113.08$

77.32

$\{77.00$

76.68

73.99

$-26.69$

$<13.44$

$-13.12$ 

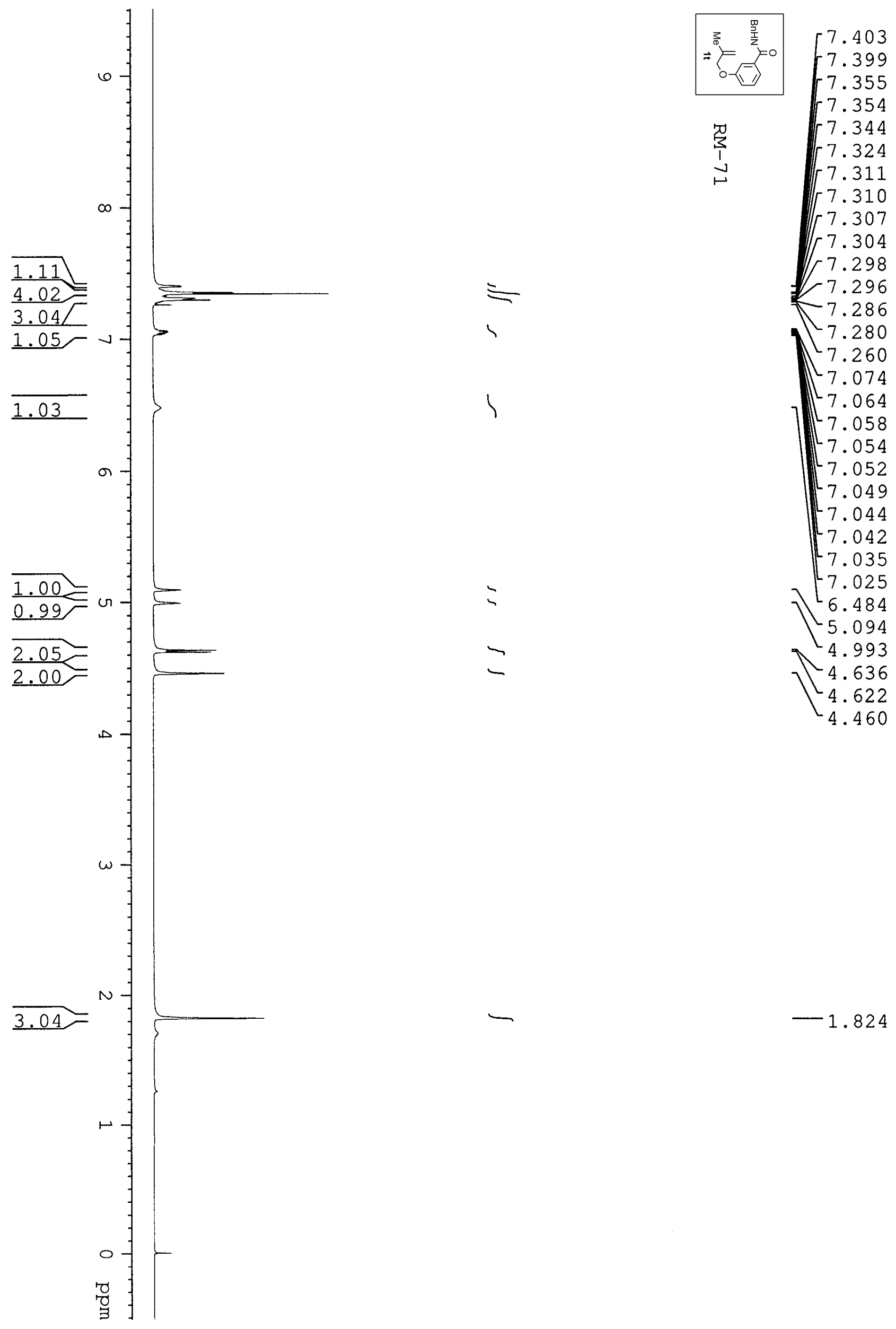

$-1.824$ 

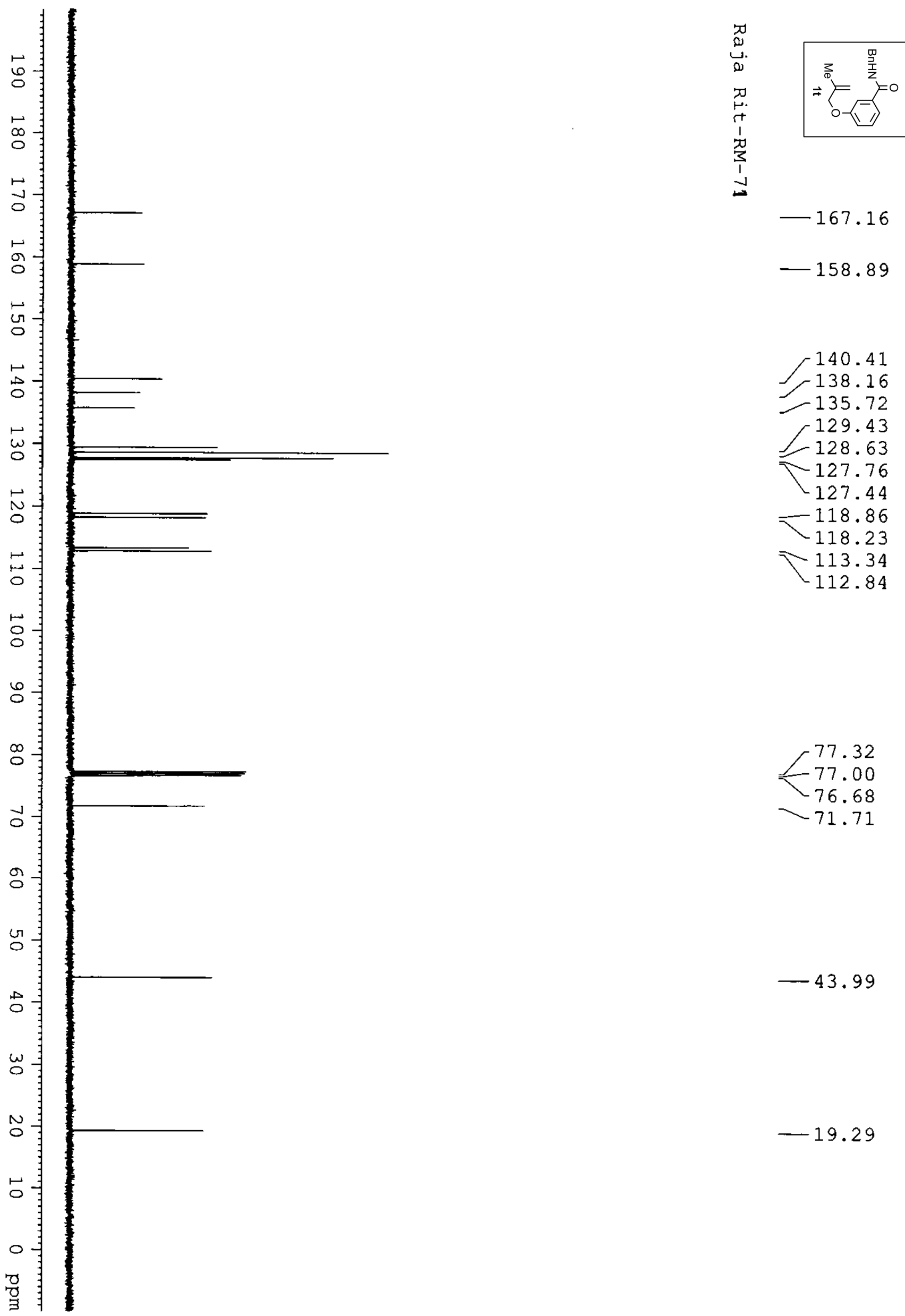

140.41

$-138.16$

$-135.72$

129.43

128.63

$-127.76$

127.44

$-118.86$

118.23

$-113.34$

$-112.84$

77.32

$-77.00$

76.68

$-71.71$

$-43.99$

$-19.29$ 


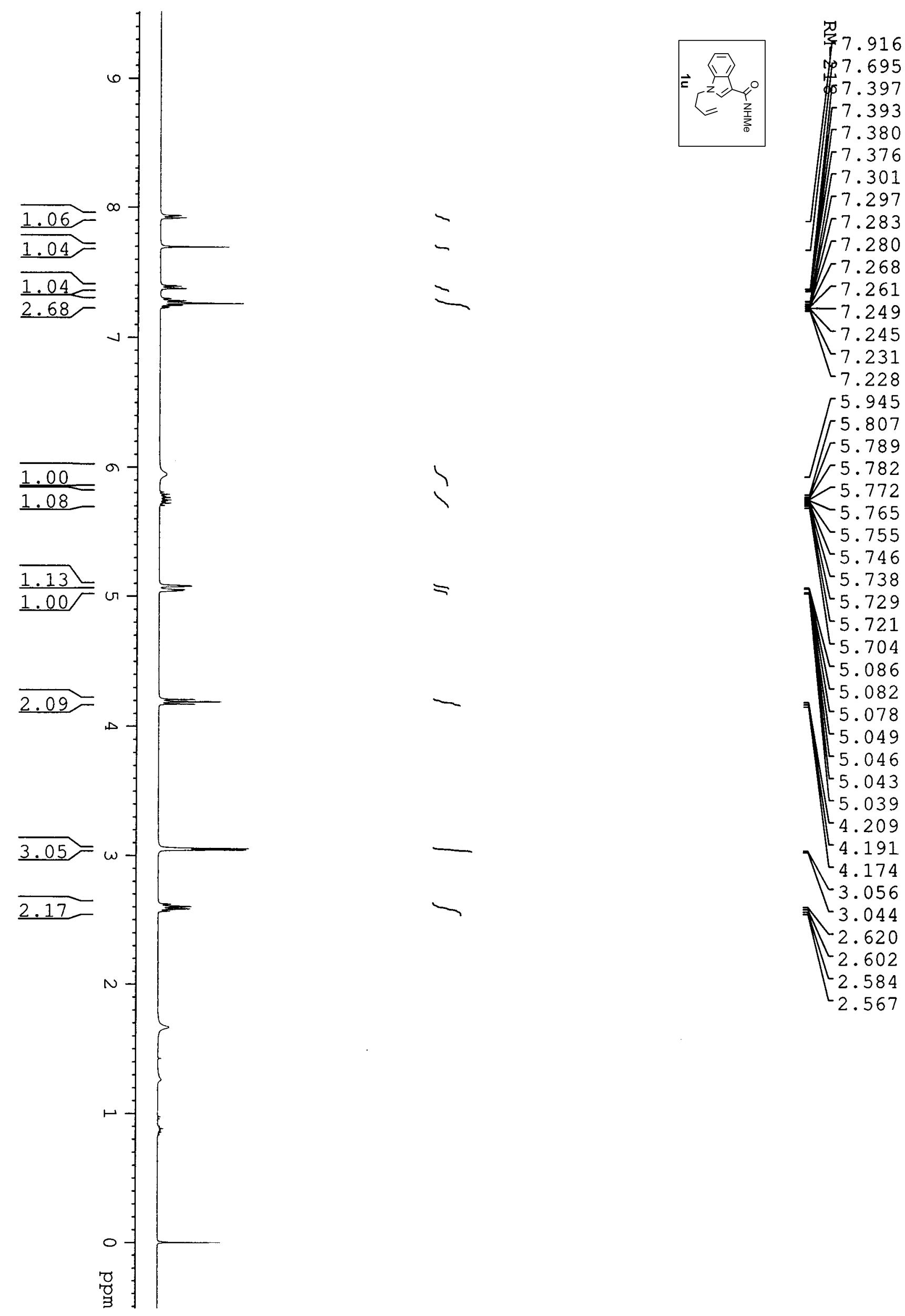




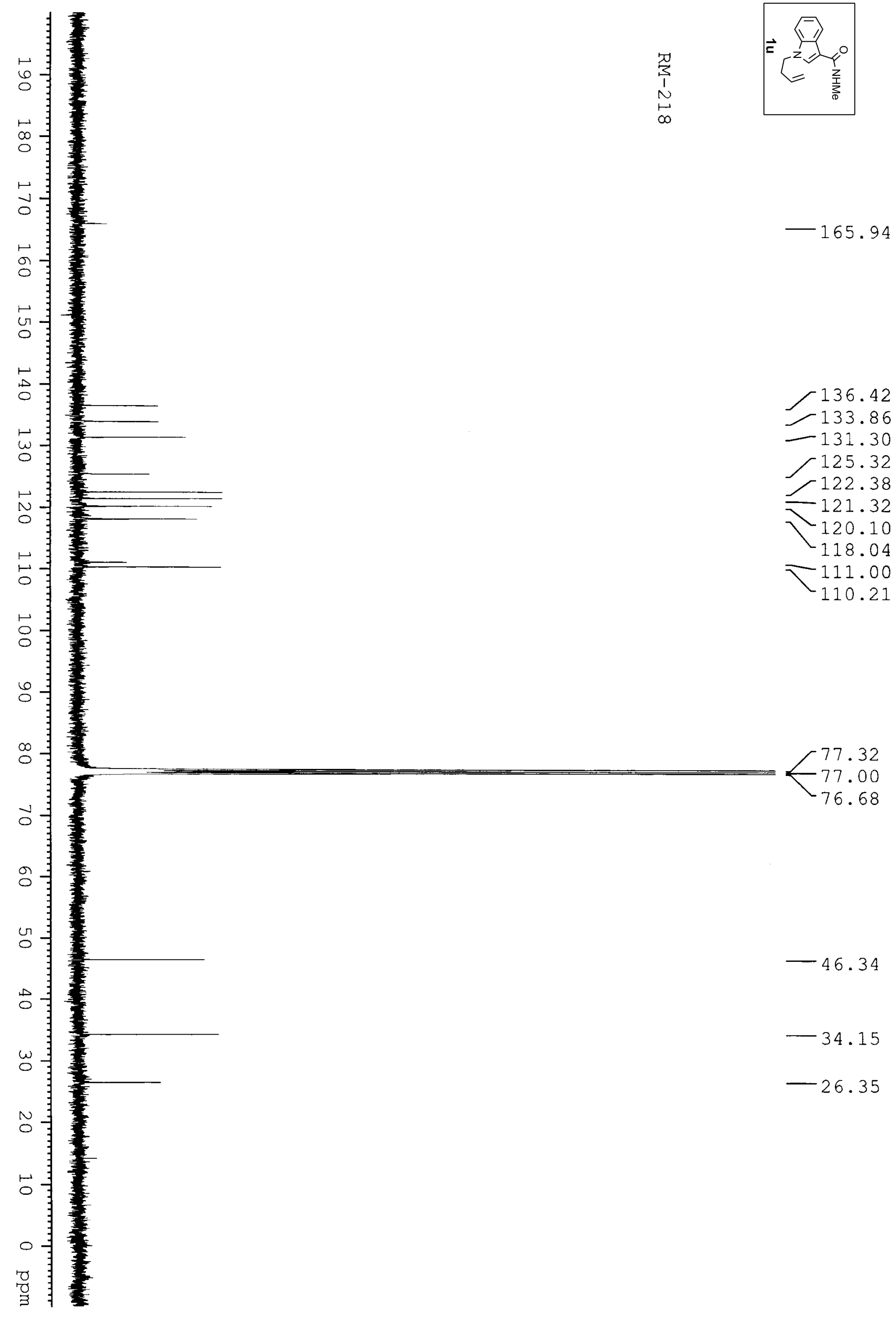



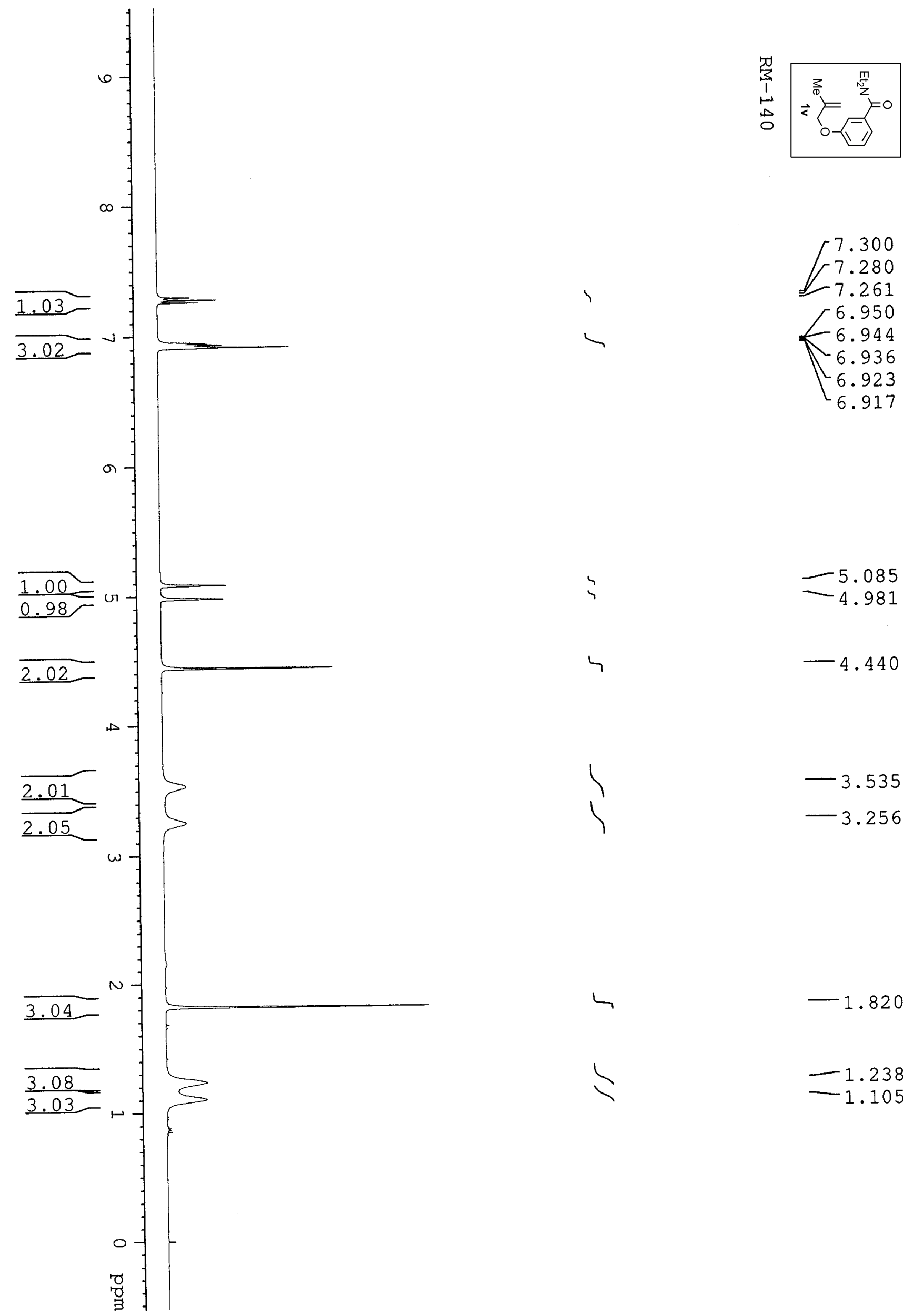

6.950

$-6.944$

$-6.936$

6.923

6.917

บ

$-5.085$

$-4.981$

4

$-4.440$

$-3.535$

$-3.256$

$\checkmark$

$-1.820$

1.238
-1.105

$\checkmark$

$-1.105$ 


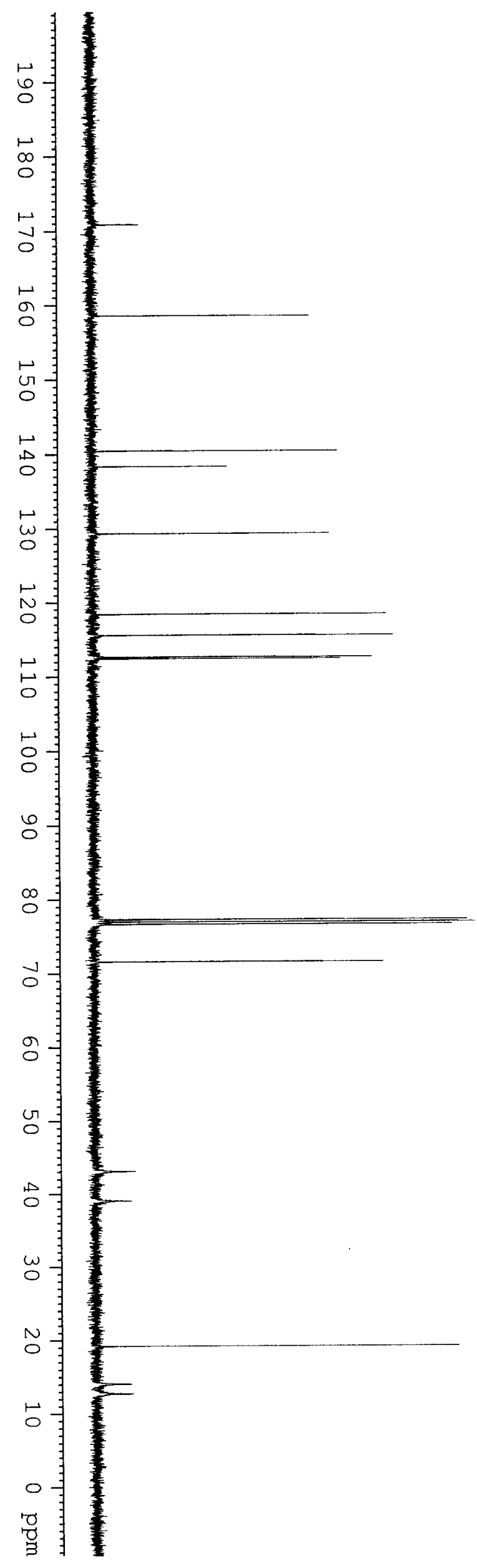

疍
1
5
0

$\overrightarrow{<}=\sum_{0}^{\frac{3}{0}}=0$

$-170.85$

$-158.55$

$-140.42$

$-138.36$

$-129.35$

$-118.37$

$-115.56$

$\lceil 112.64$

$-112.42$

77.32

$\leftarrow 77.00$

75.68
-71.59

71.59

$-43.07$

39.04

$-19.20$

$-14.07$

$-12.72$ 


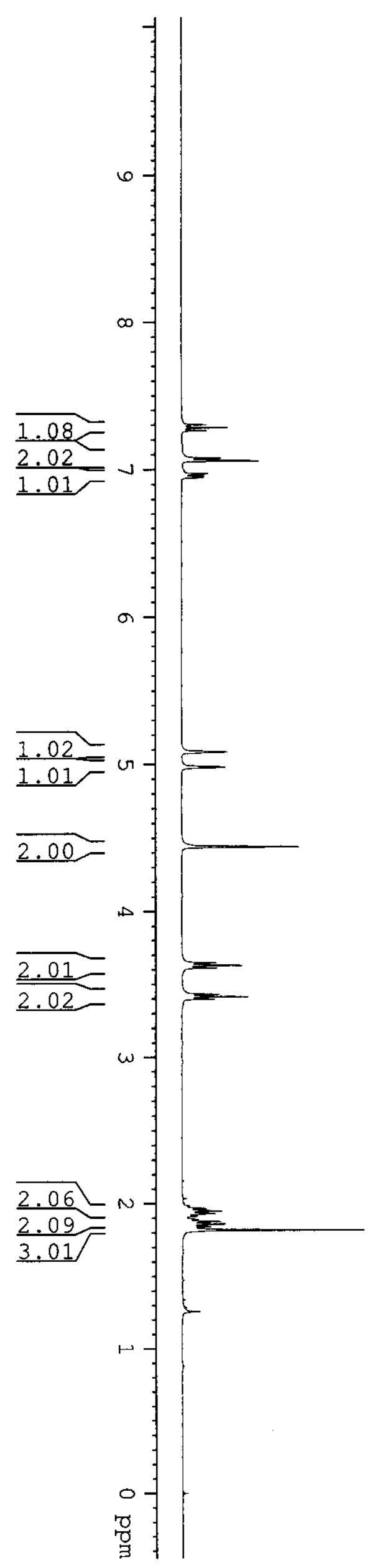

2
3
1
$\infty$
$\infty$
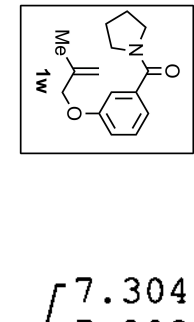

7.283

7.263

7.077

$\sum^{2}$

7.061

7.057

$-6.973$

$-6.967$

6.954

6.949

6.945

$\zeta$

-5.085
-4.982

4.982

$\longrightarrow \quad-4.441$

3.647

3.630

7

3.612

3.431

3.414

3.398

1.966

1.950

1.932

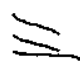

1.916

1.892

1.877

1.860

1.844

1.819 

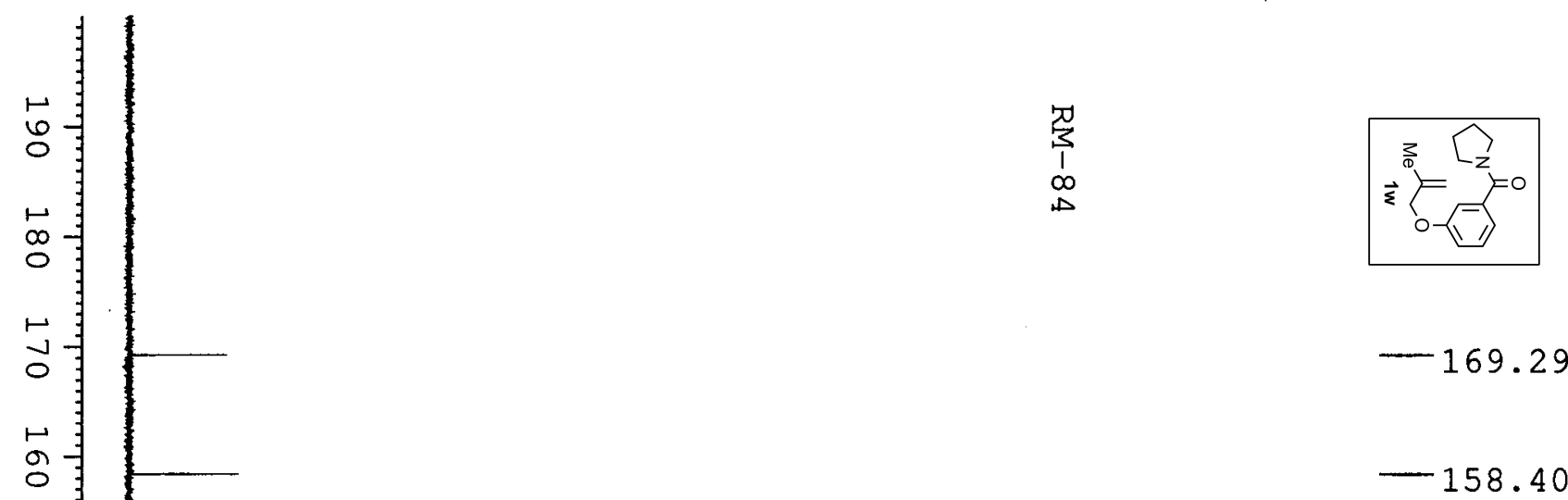

$-140.42$

$-138.22$

$-129.14$

$-119.20$

116.30

$-113.13$

官

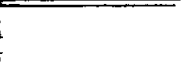

응

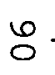

$\infty$

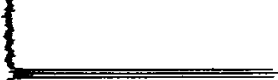

77.32
$\leftarrow 77.00$
76.68
71.58

o

r

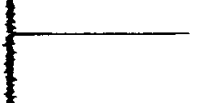

$-49.40$

$-45.98$

$\stackrel{\leftrightarrow}{\circ}$

$\omega$

瓷

$-26.16$

$-24.25$

$-19.19$

\begin{tabular}{l}
5 \\
0 \\
0 \\
7 \\
\hline
\end{tabular} 

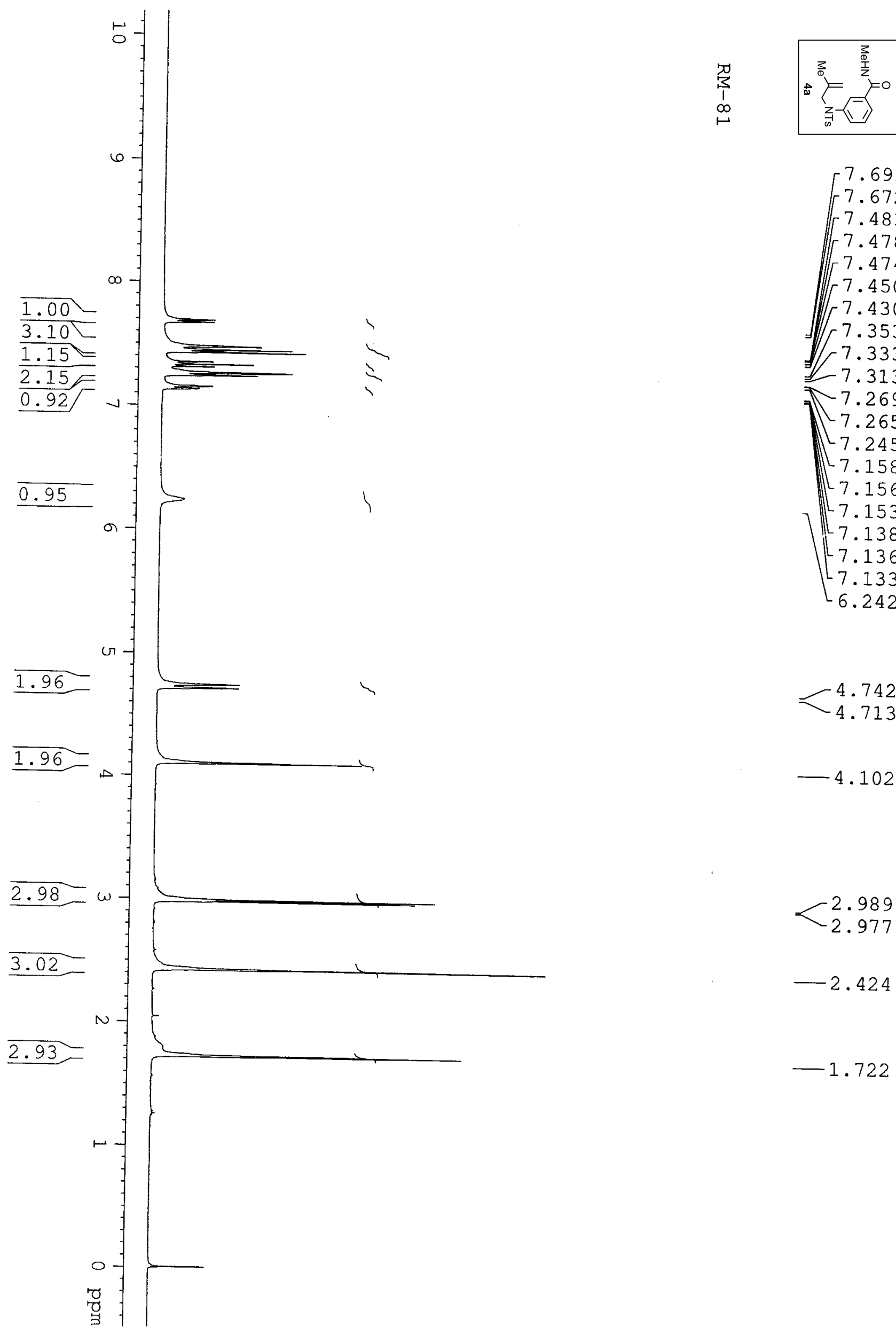

7.691

$-7.672$

7.483

7.478

7.474

7.450

7.430

. 7.353

7.333

$-7.313$

$-7.269$

$-7.265$

$-7.245$

7.158

7.156

7.153

7.138

$-7.136$

7.133

6.242

4.742

4.713

$-4.102$

$-2.989$

$-2.977$

$-2.424$

$-1.722$ 


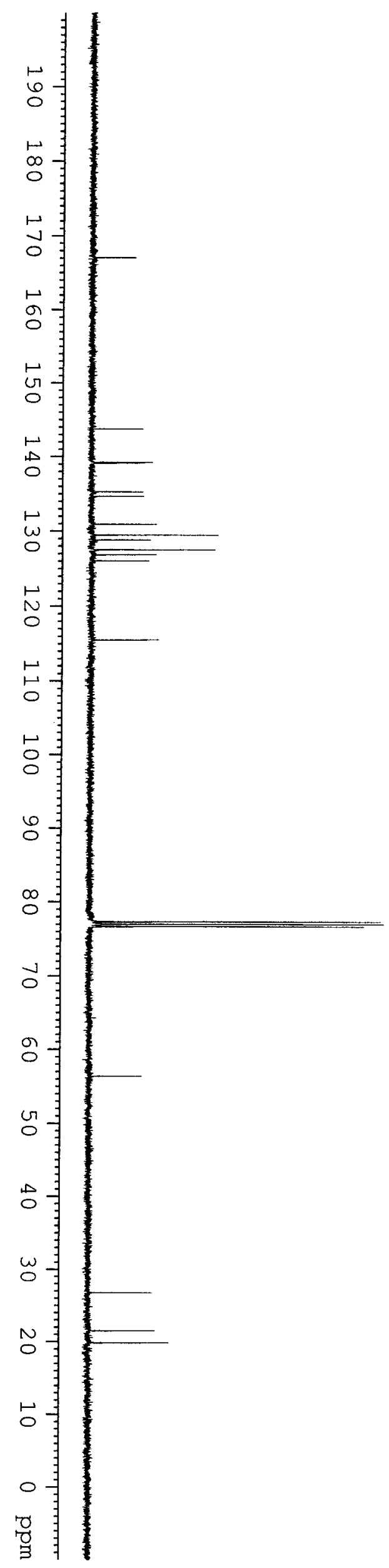

2
3
1
$\infty$

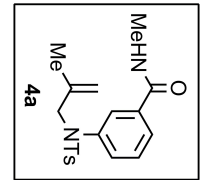

$-167.10$

$\Gamma \begin{aligned} & 143.76 \\ & 139.25\end{aligned}$

$\sqrt{139.12}$

135.28

$-134.69$

130.97

$-129.52$

128.85

$-127.55$

126.88

126.07

115.50

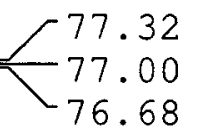

$-56.38$

$-26.76$

$-21.48$

$-19.83$ 


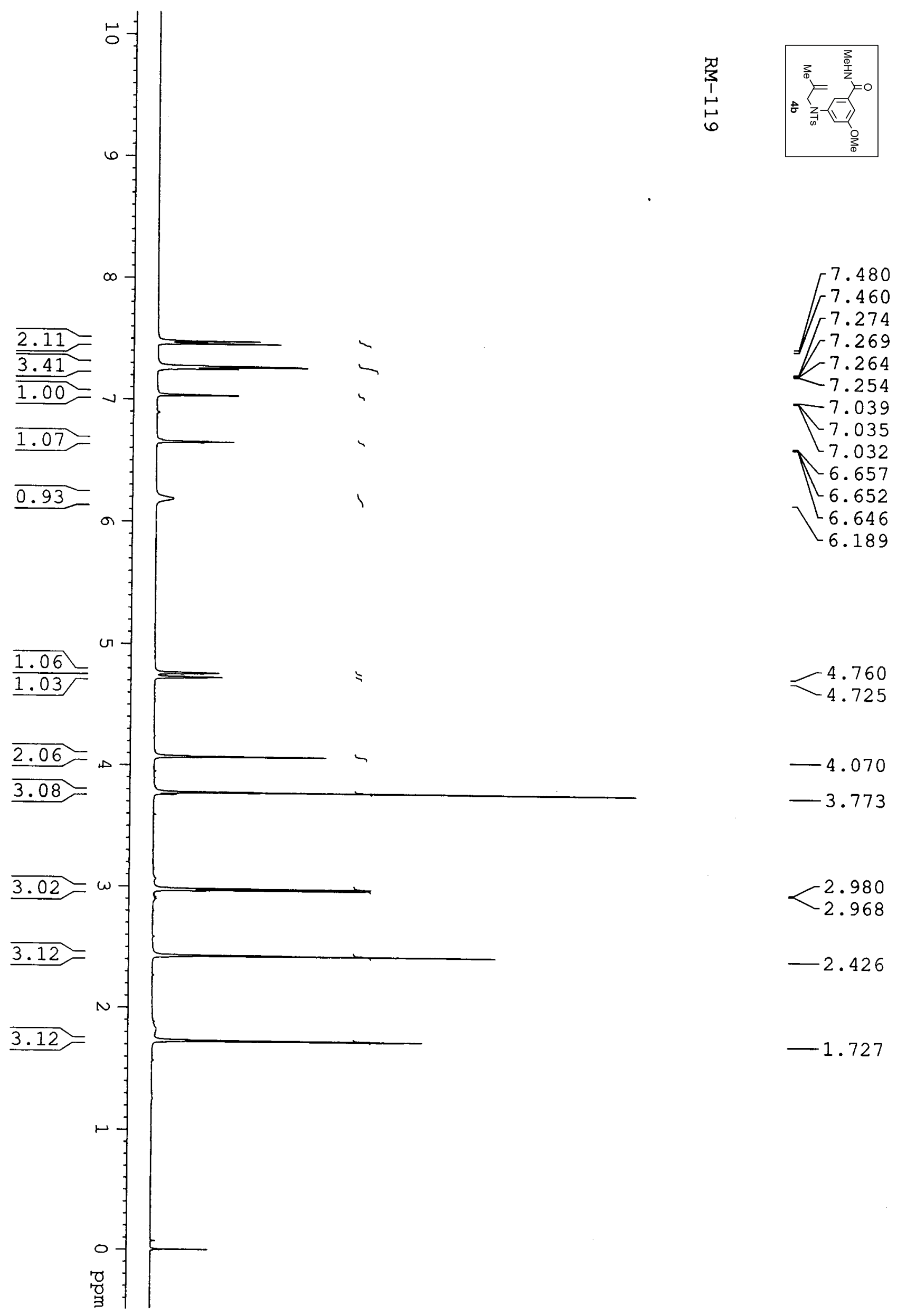



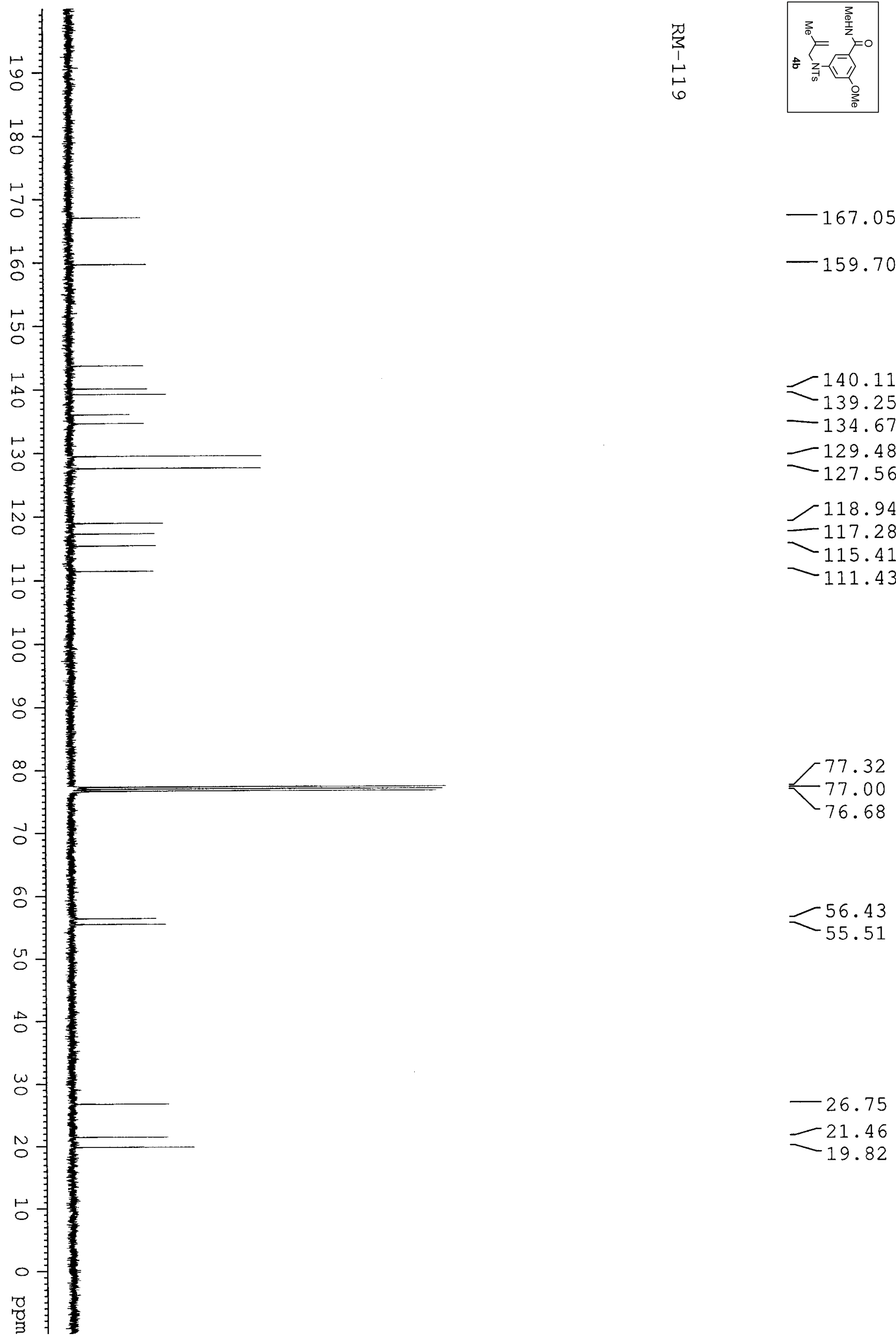

$-159.70$

$-140.11$

$-139.25$

$-134.67$

$-129.48$

$-127.56$

$-118.94$

$-117.28$

$-115.41$

$-111.43$

77.32

$-77.00$

$-76.68$

$-56.43$

55.51

$-26.75$

$-21.46$

$-19.82$ 

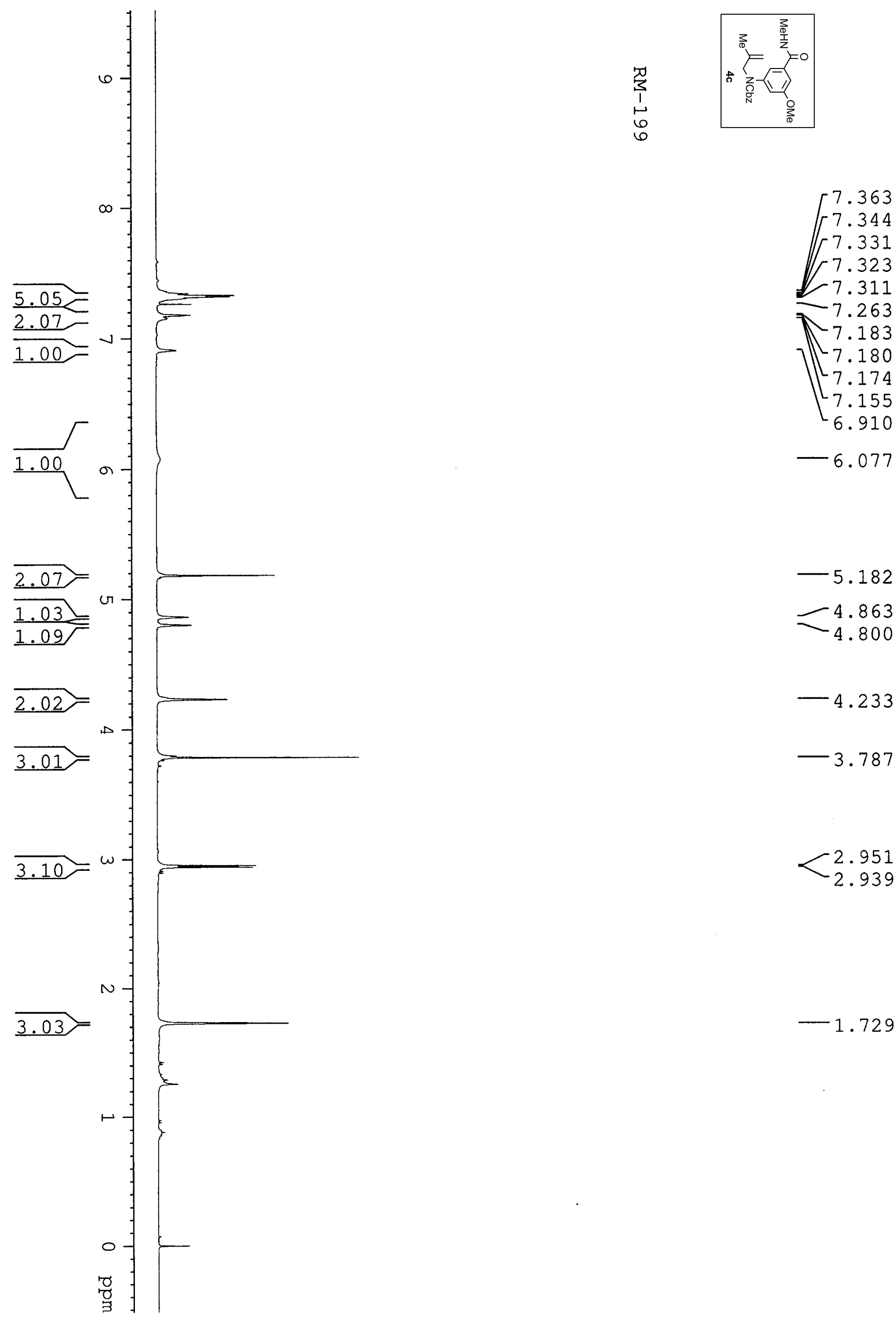

$-5.182$

4.863
-4.800

$-4.233$

$-3.787$

951

$-2.939$

$-1.729$ 


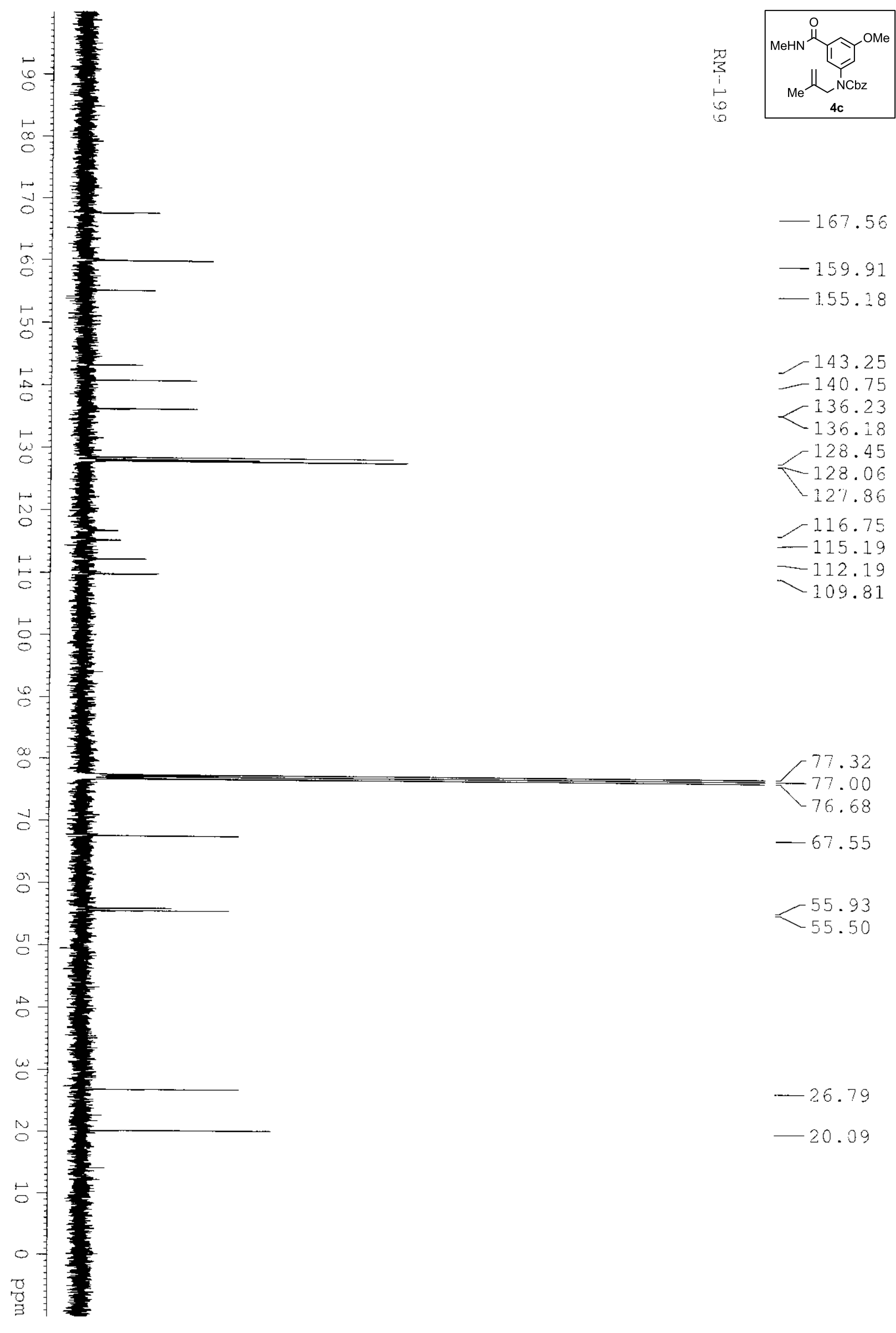




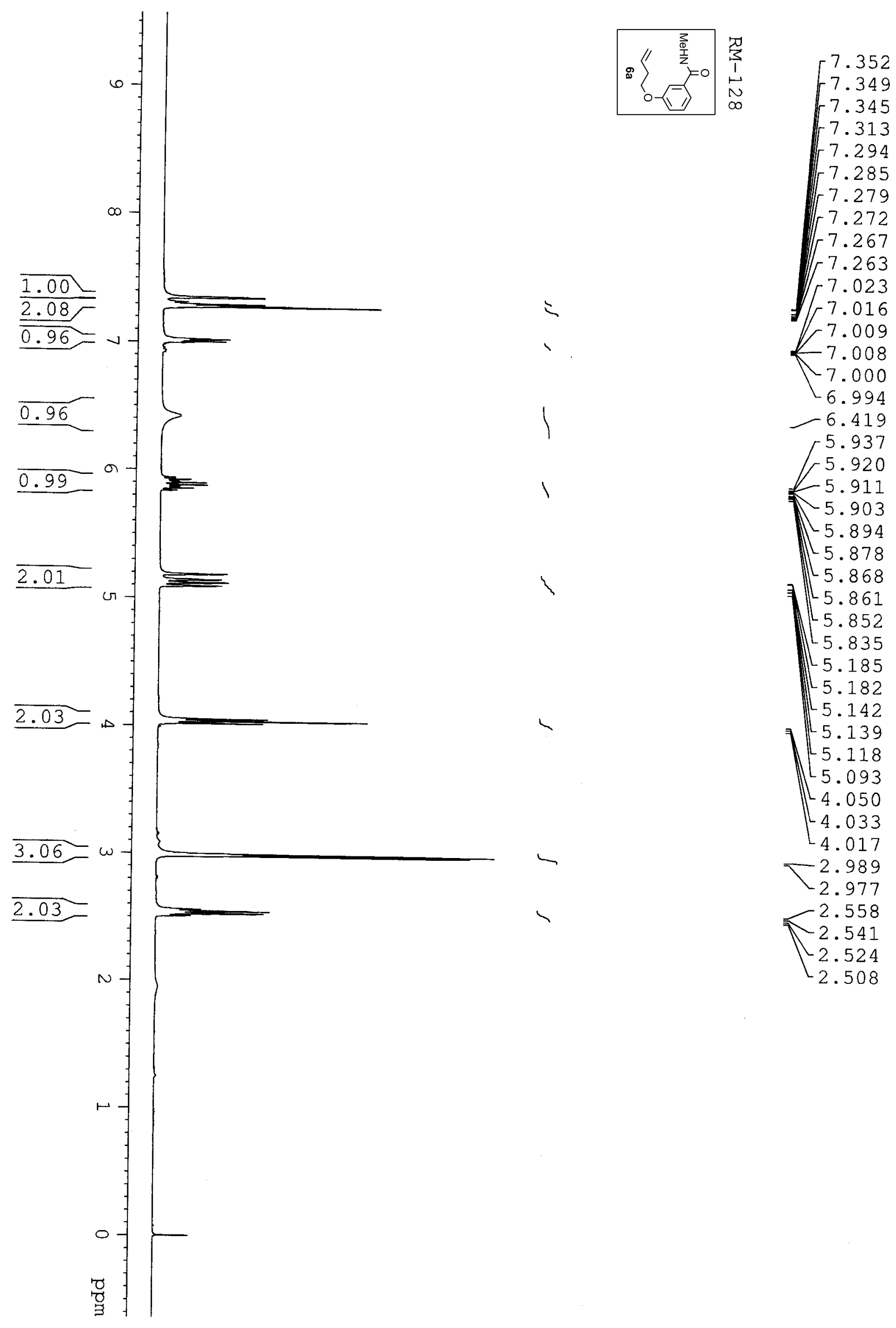



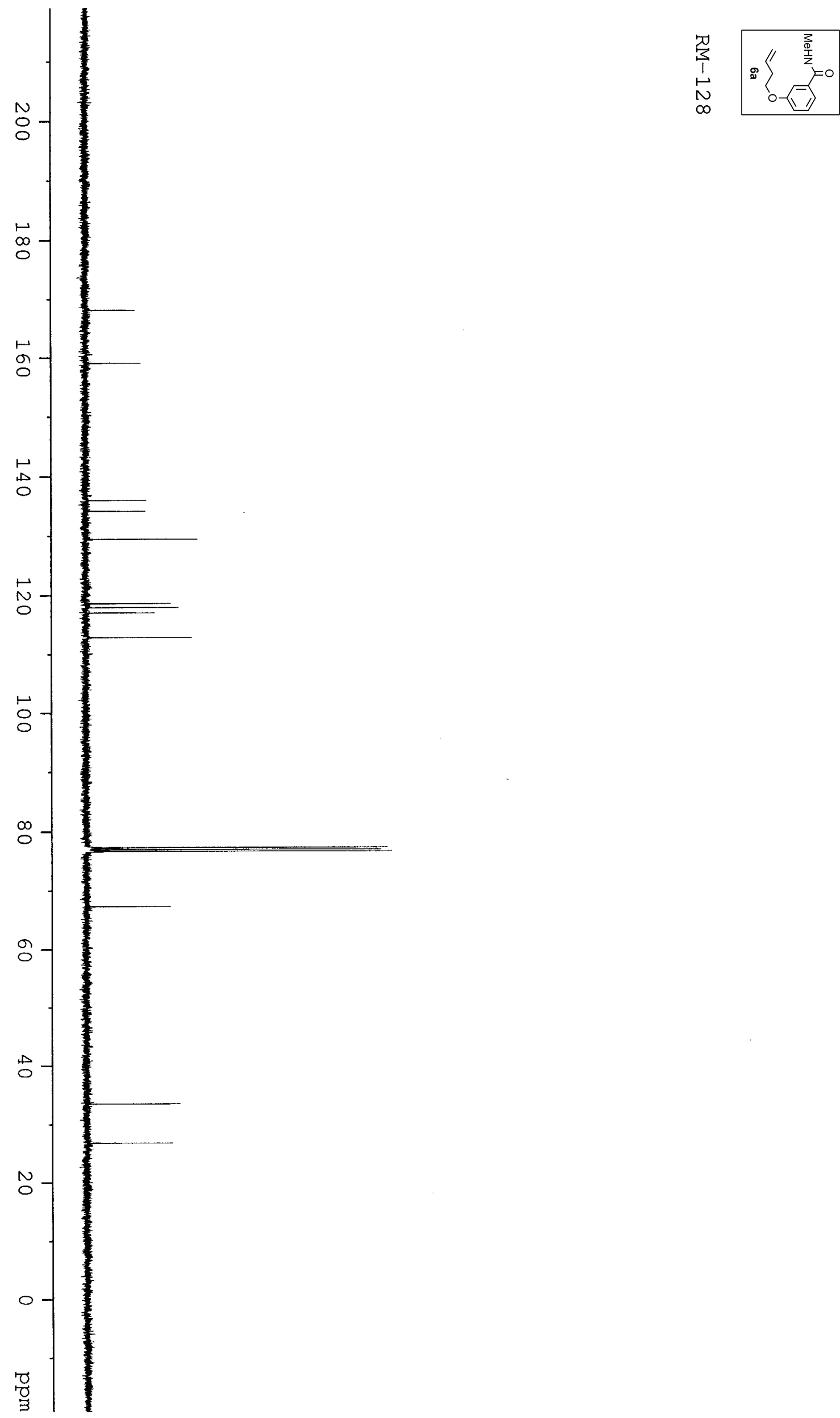

$-168.08$

$-159.05$

$-136.01$

$-134.20$

$-129.45$

118.65

$=117.97$

117.08

112.89

77.32

$\leftarrow 77.00$

76.68

$-67.29$

$-33.48$

$-26.79$ 

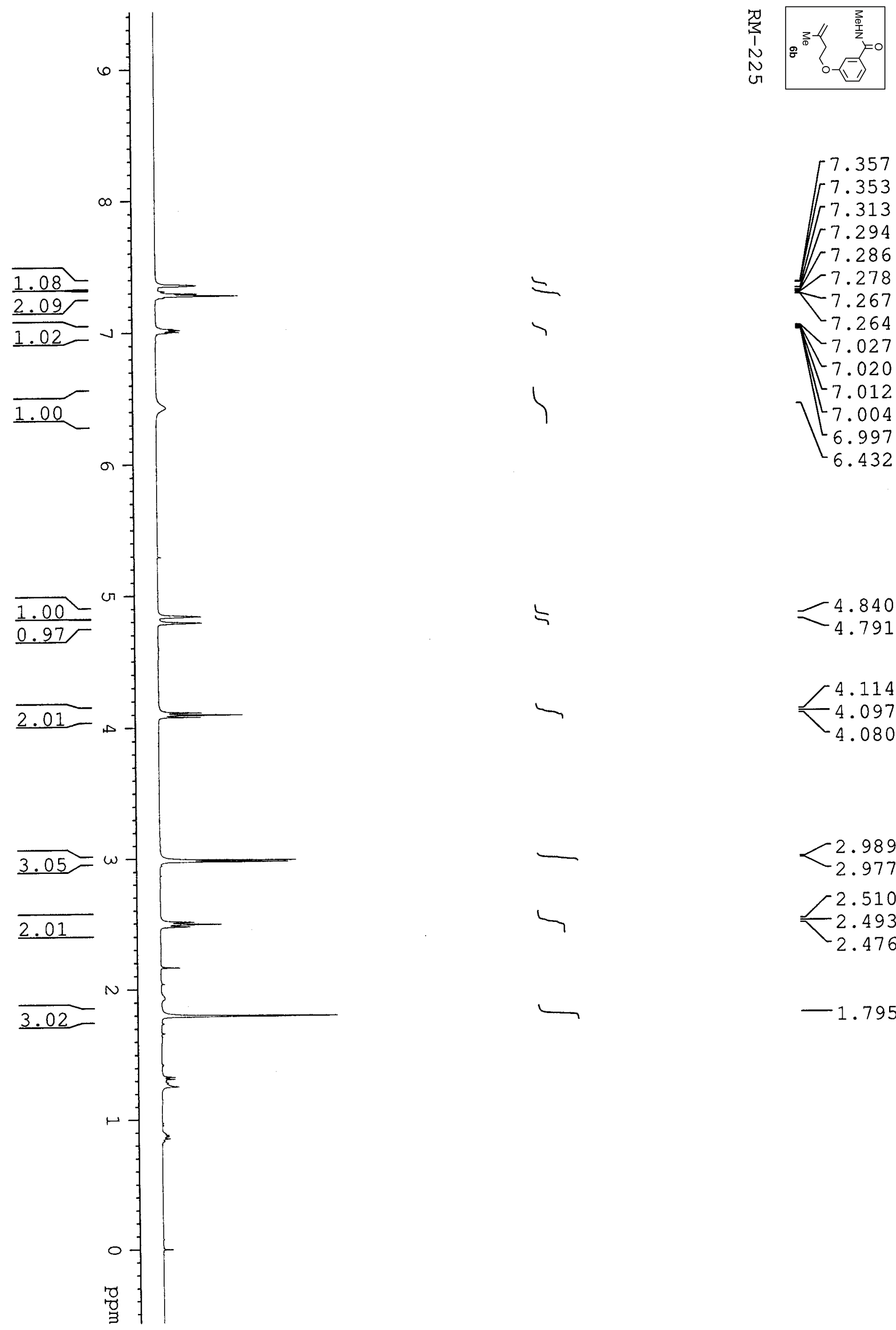

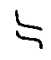

4.840

$-4.791$

.114

4.014
4.097
4.080

2.989
-2.977

2.510

2.493
-2.476

2.476

$-1.795$ 

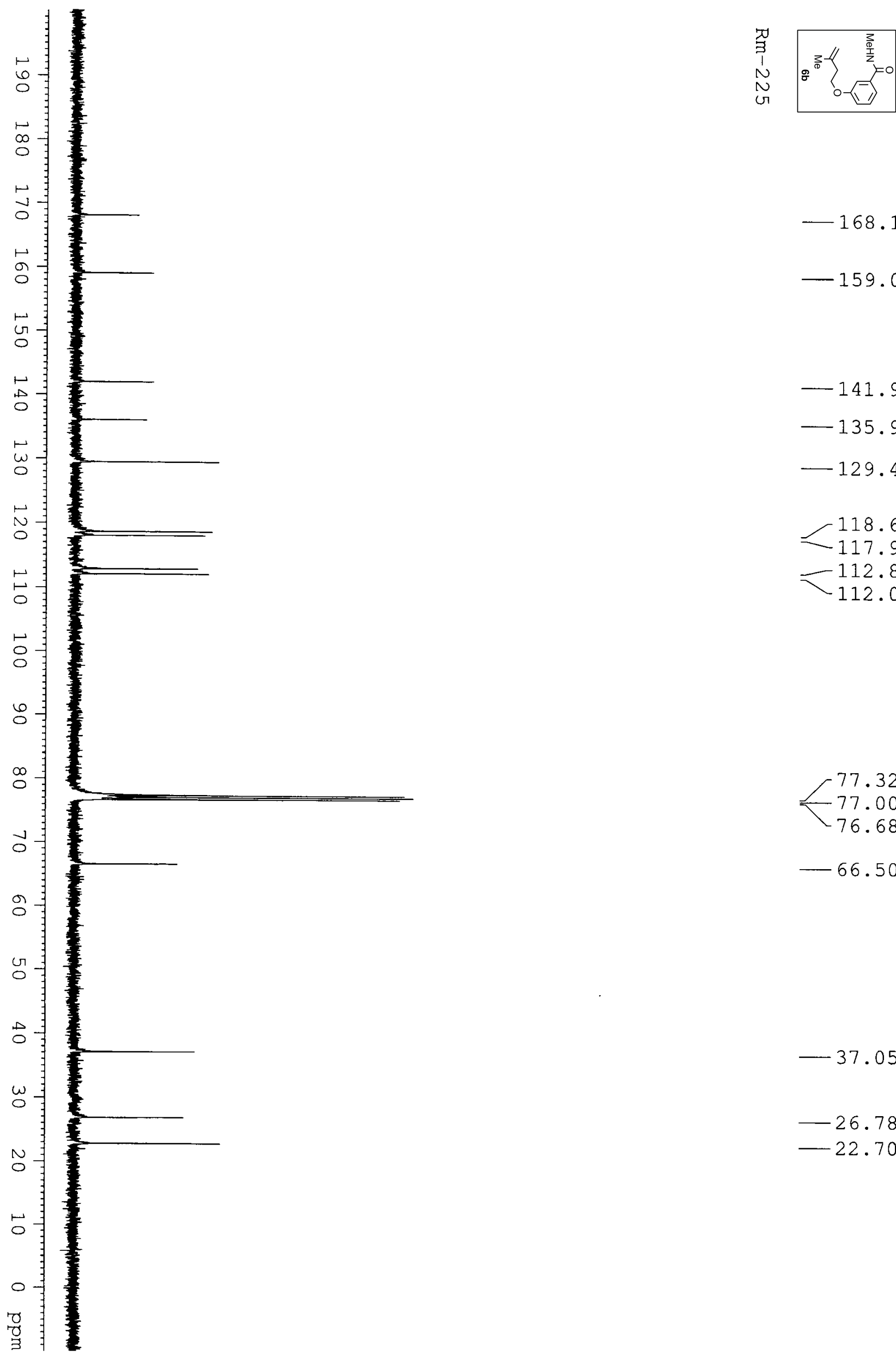

$-168.10$

$-159.05$

$-141.95$

$-135.99$

$-129.44$

$-118.62$

$-117.99$

$-112.86$

$-112.04$

$-77.32$

77.00

76.68

$-66.50$

$-37.05$

$-26.78$

$-22.70$ 

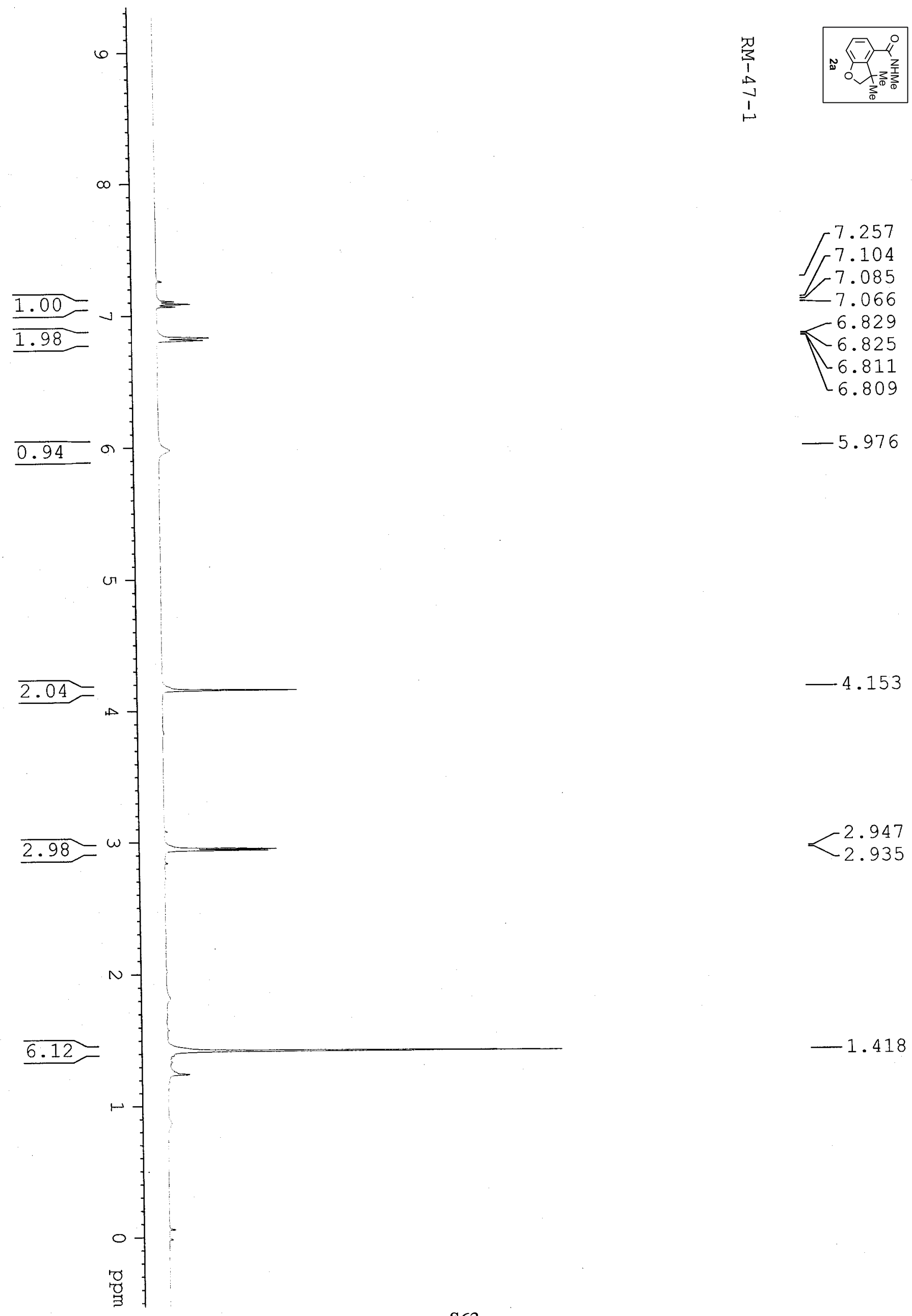
$-6.829$

$-6.825$

$-6.811$

6.809

$-5.976$

$-4.153$

$-2.947$

2.935

$-1.418$ 


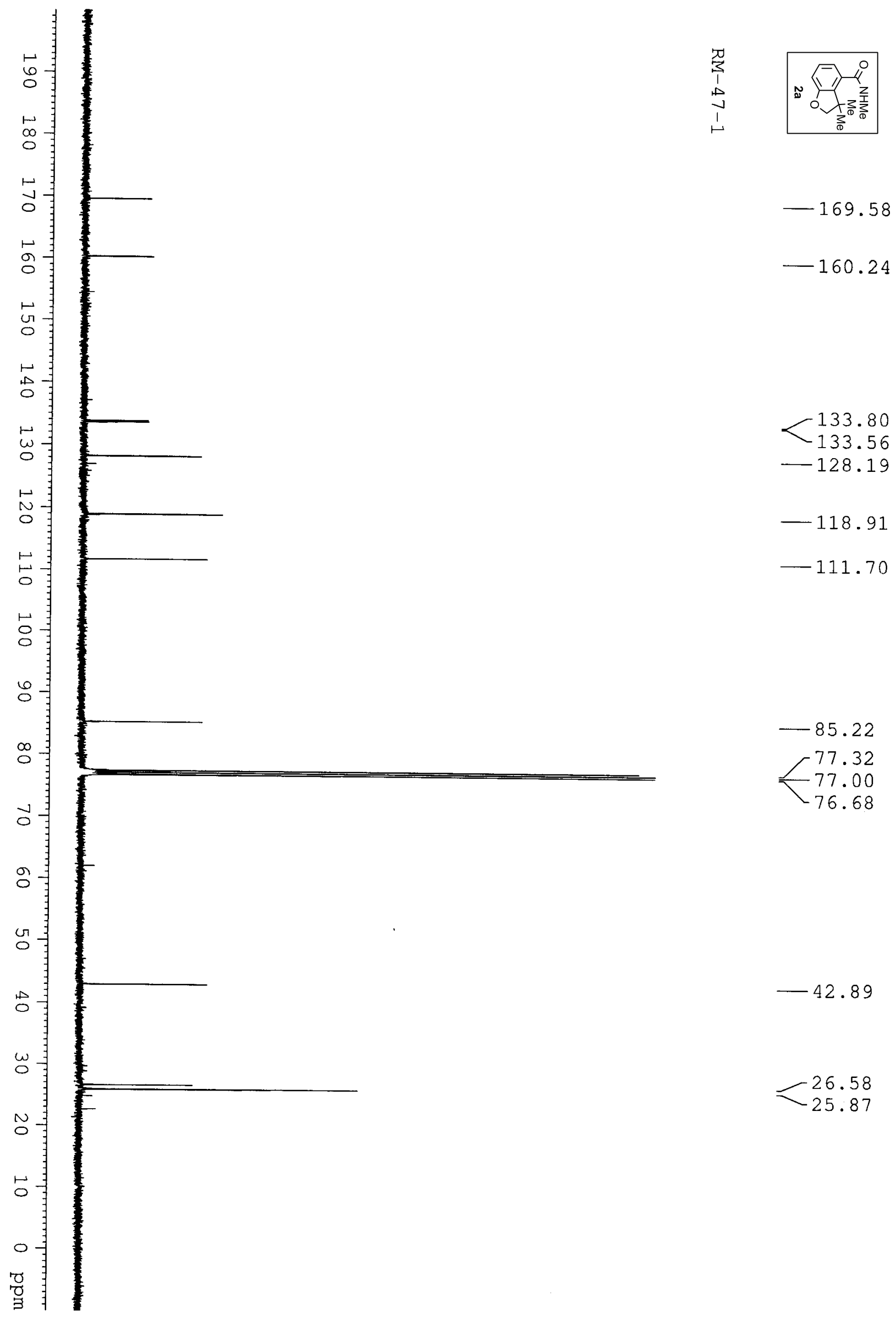




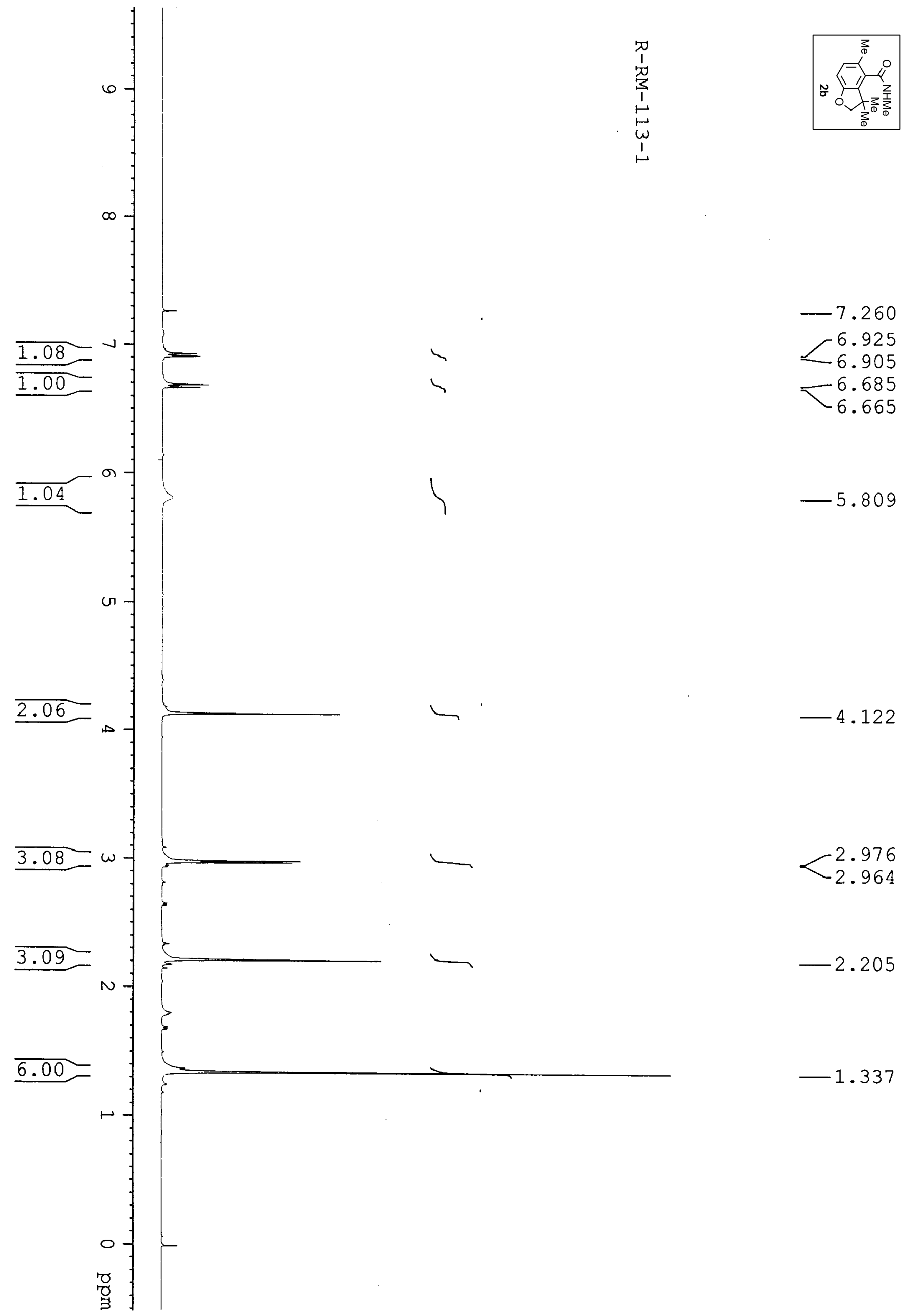




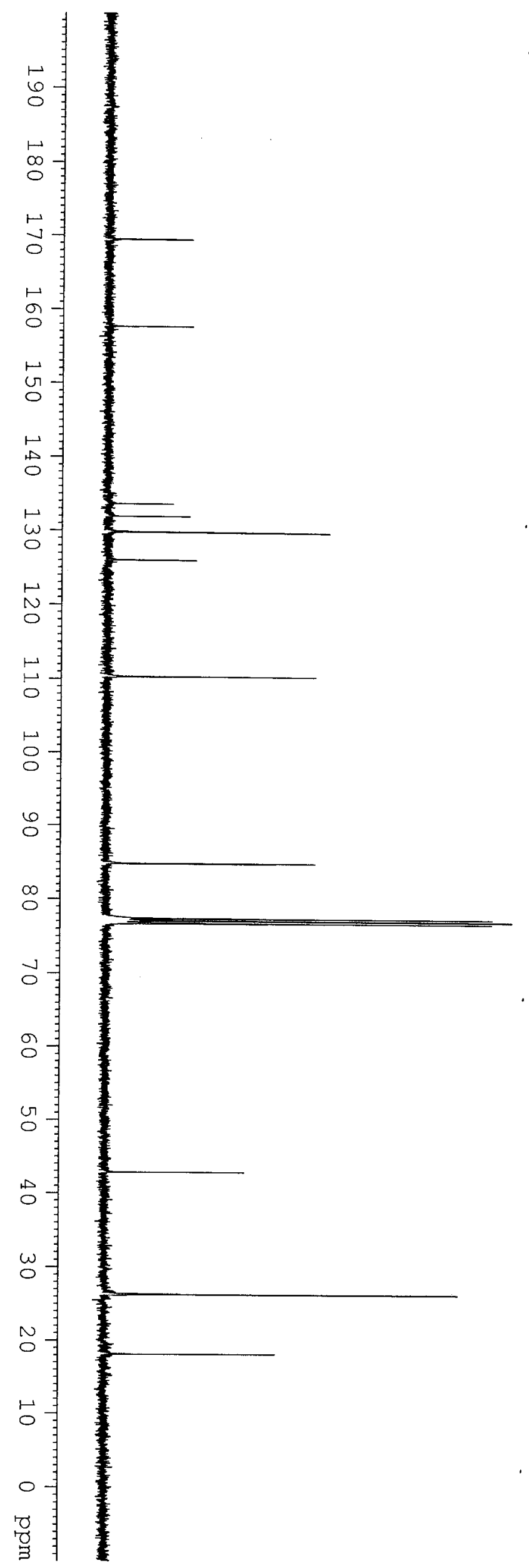

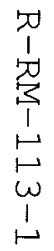

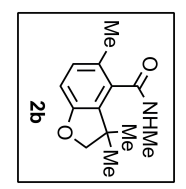

$-169.49$

$-157.65$

$-133.64$

$-131.93$

$-129.80$

$-125.98$

$-110.31$

$-84.78$

77.32

$-77.00$

76.68

$-42.77$

$-26.22$

$-26.17$

$-18.04$ 


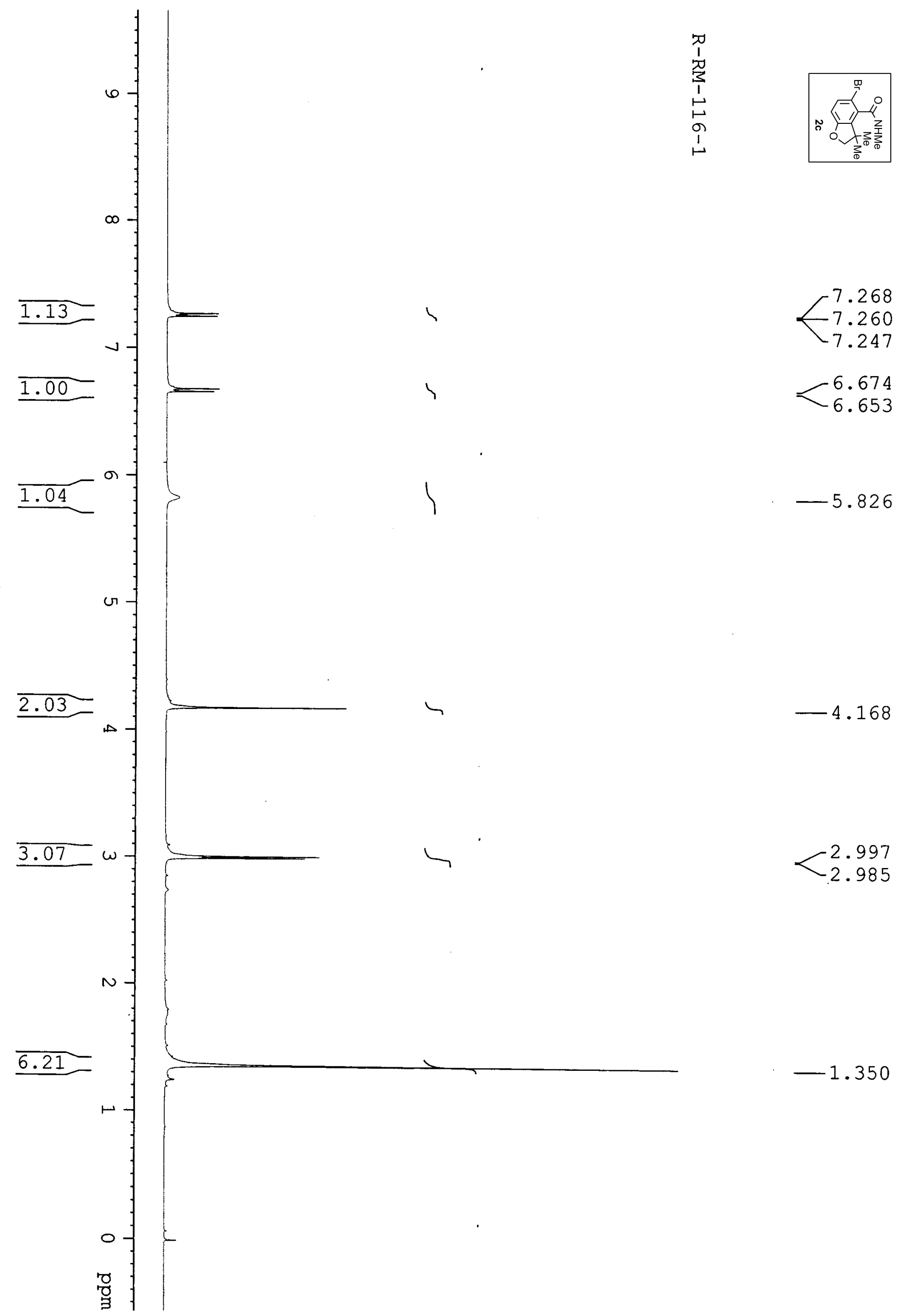




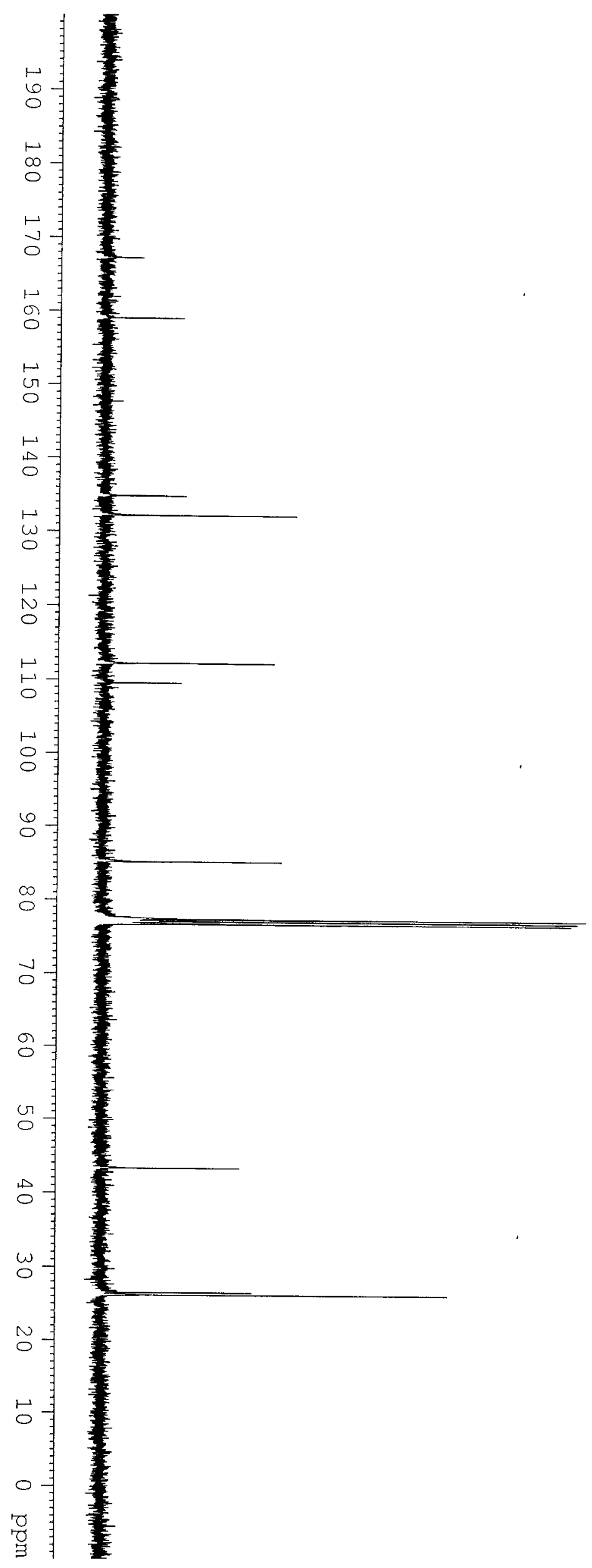

00
1
0
3
1
5
5
0
1
$⺊$

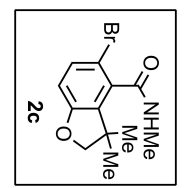

$-167.27$

$-159.04$

134.84

$-134.77$

$\checkmark 132.17$

-112.21
-109.55

$-85.13$

$-77.32$

$-77.00$

$-76.68$

$-43.23$

26.41
-26.05

$-26.05$ 


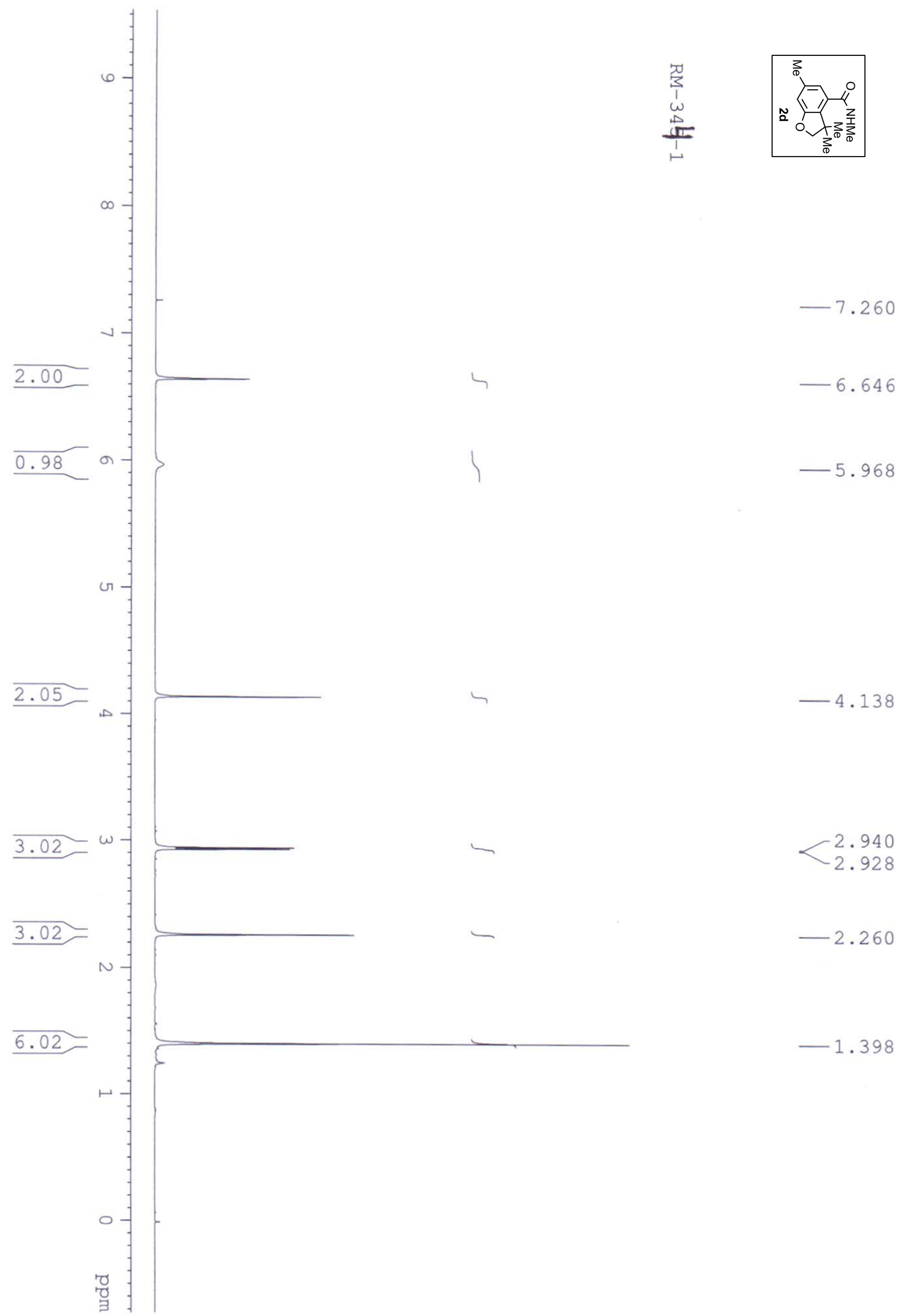



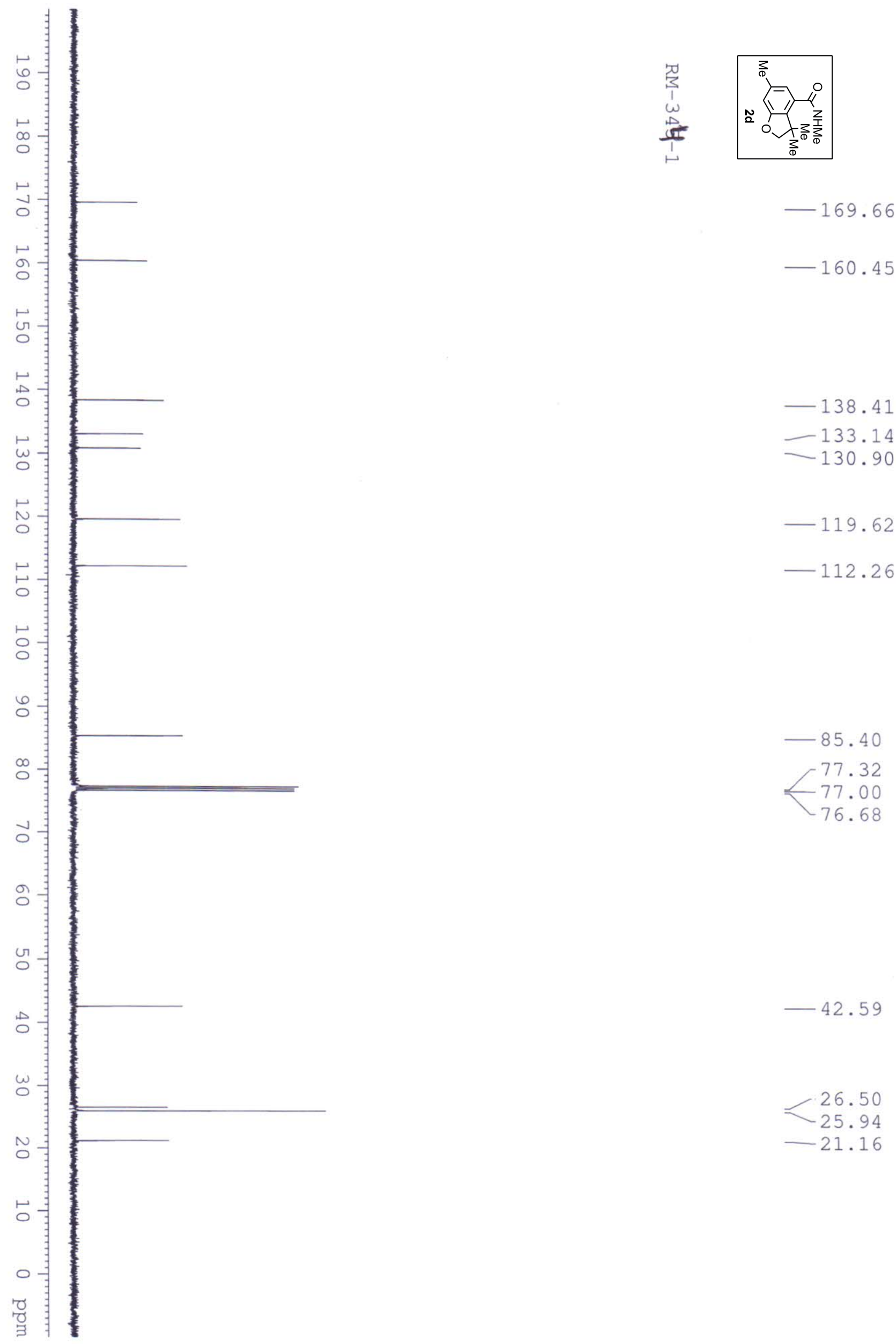

$-138.41$

$-133.14$

$-130.90$

$-119.62$

$-112.26$

$-85.40$

$-77.32$

$-77.00$

$-76.68$

$-42.59$

26.50
-25.94
-21.16 


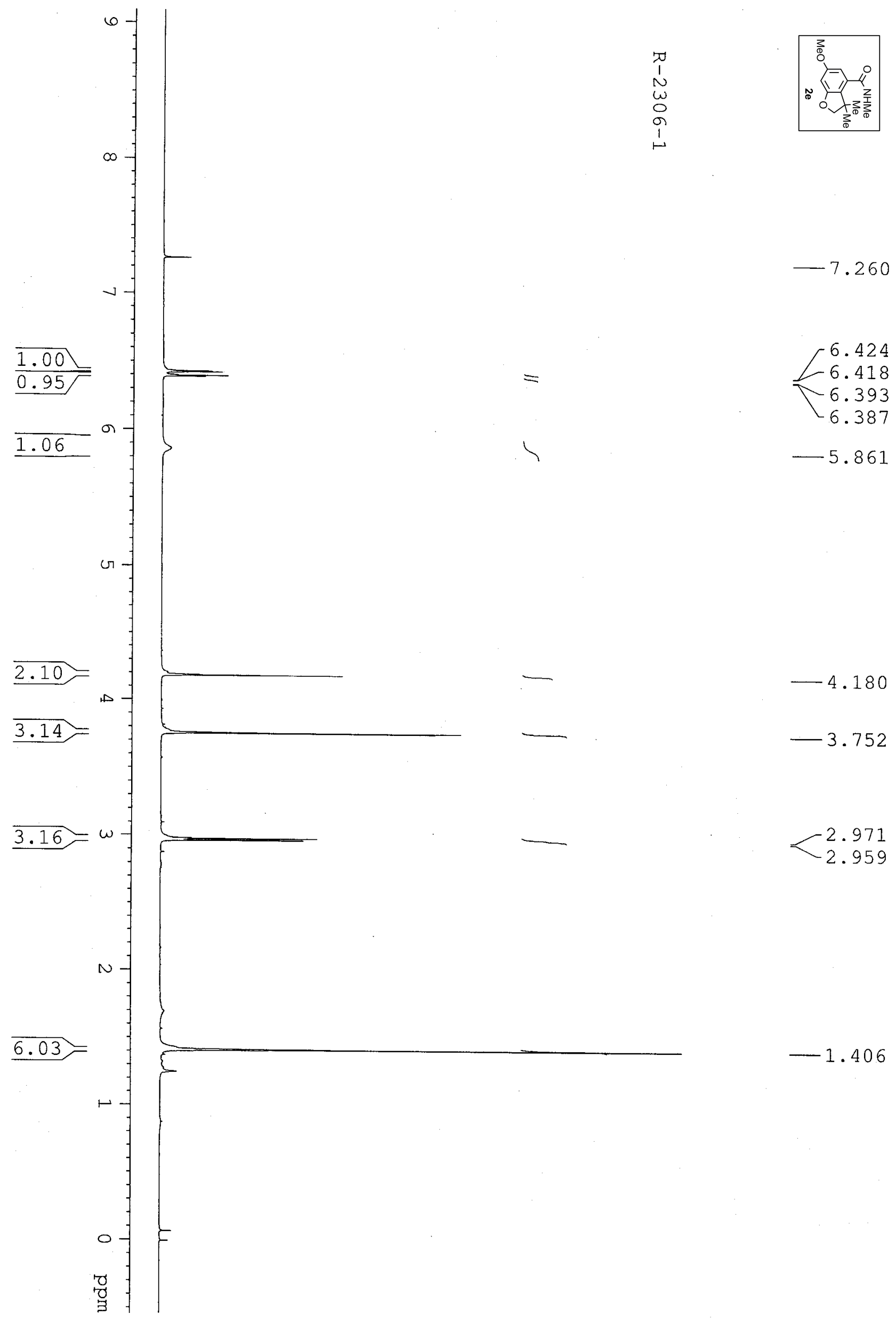



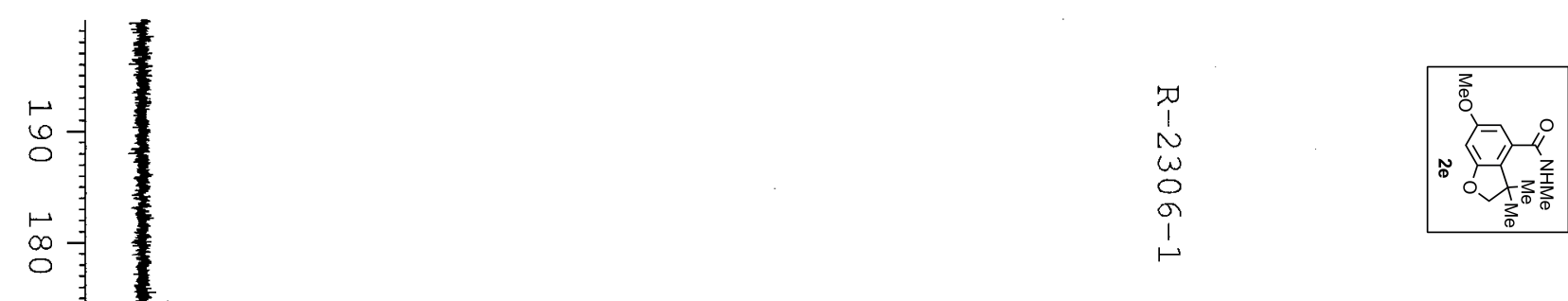

$-169.38$

$-161.63$

o

○े

$\checkmark$

o

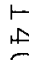

$\ddot{\omega}$

$\stackrel{\leftrightarrow}{N}$

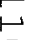

$\circ$

6

$\circ$

o

ᄋ

o

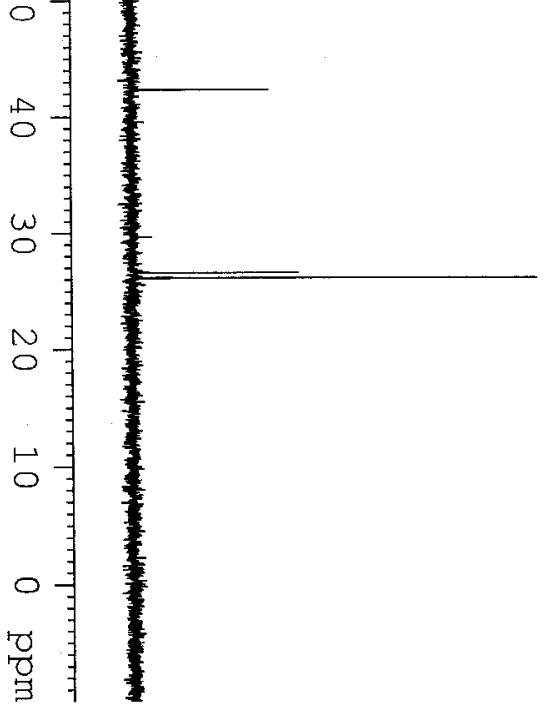

$-104.87$

$-97.88$

$-85.98$

$-77.32$

$\leftarrow-77.00$

$-76.68$

$-55.59$

$-42.37$

26.59
26.13 


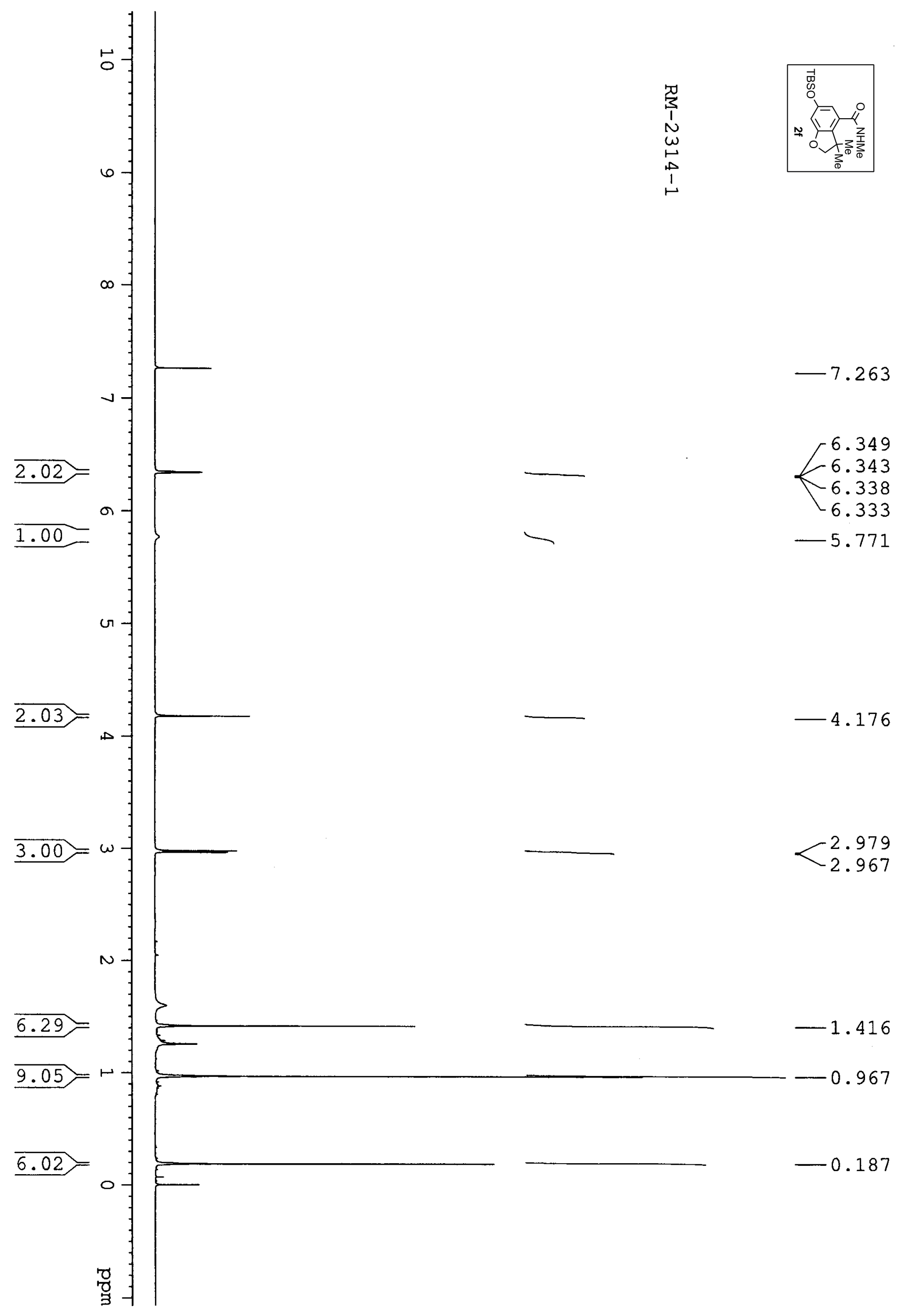



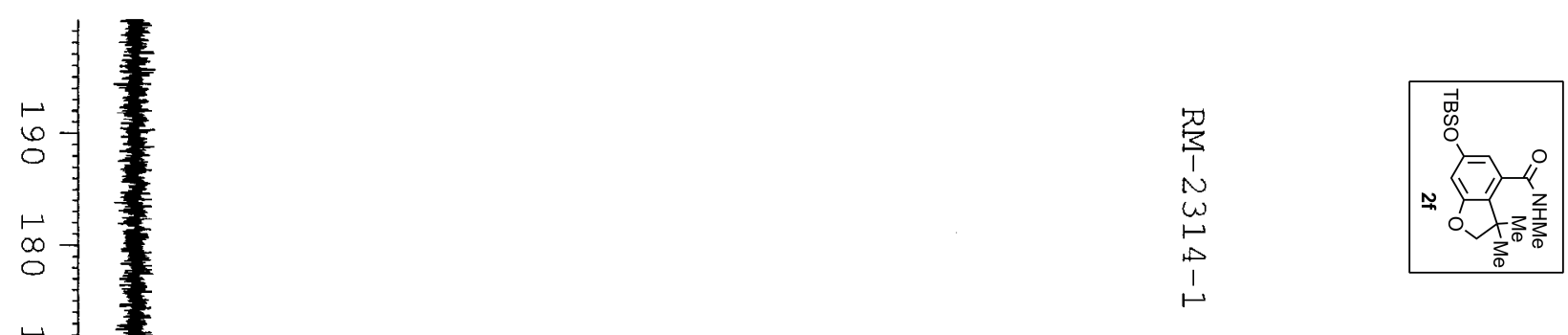

$-169.42$

$-161.43$

$-155.83$

$-133.48$

$-126.66$

$-110.69$

$-103.92$

○

$\bullet$

$\infty$

Ј

o

G

$\stackrel{\circ}{\circ}$

$-42.43$

$\omega$

$\frac{5}{3}$

임

th

6.62

26.17

25.60

18.10

○

青旁

8
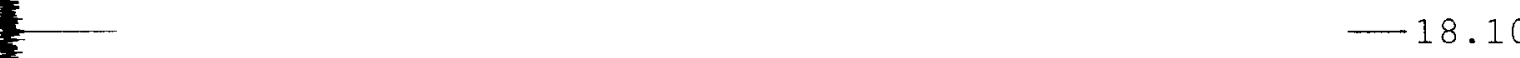

$-4.45$ 


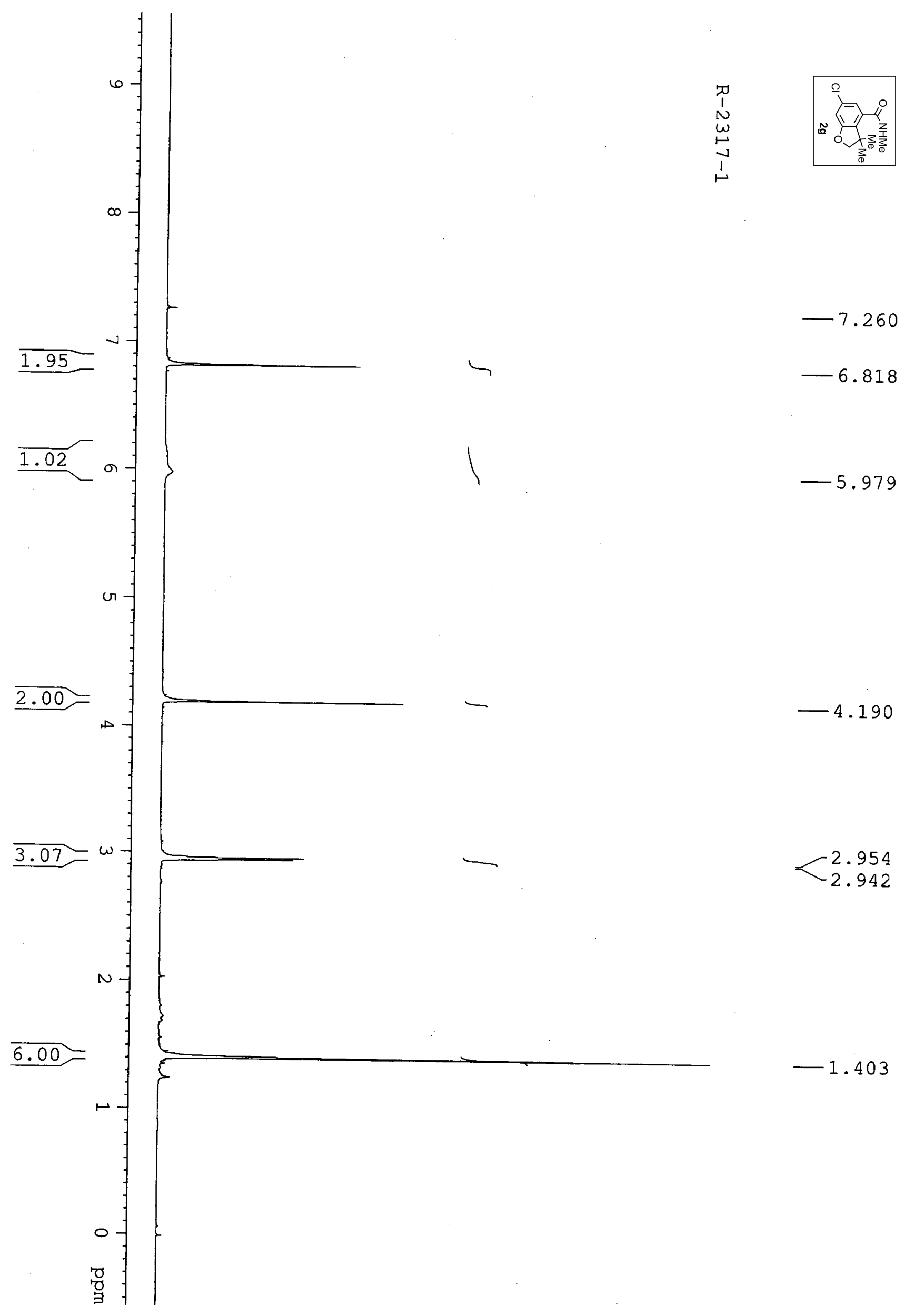



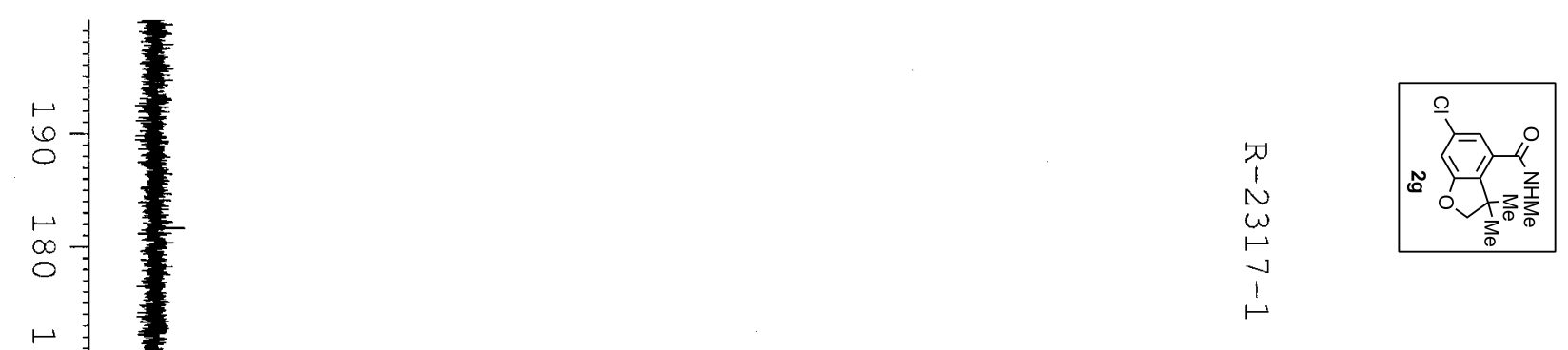

$-168.20$

$-161.19$

o

宊

$\stackrel{\circ}{\circ}$

$\stackrel{\omega}{\omega}$

134.12

$-133.30$

$-132.69$

$-118.95$

$-112.17$

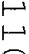

今

$\circ$

6

○

Jे

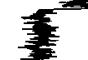

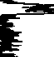

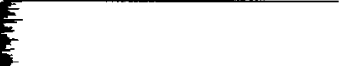

$-85.88$

77.32

$-77.00$

76.68

9

o

$\stackrel{\circ}{\circ}$

$-42.63$

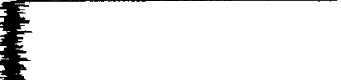

w

ㅇ.

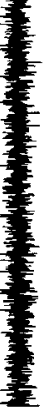

-26.62
-25.80 


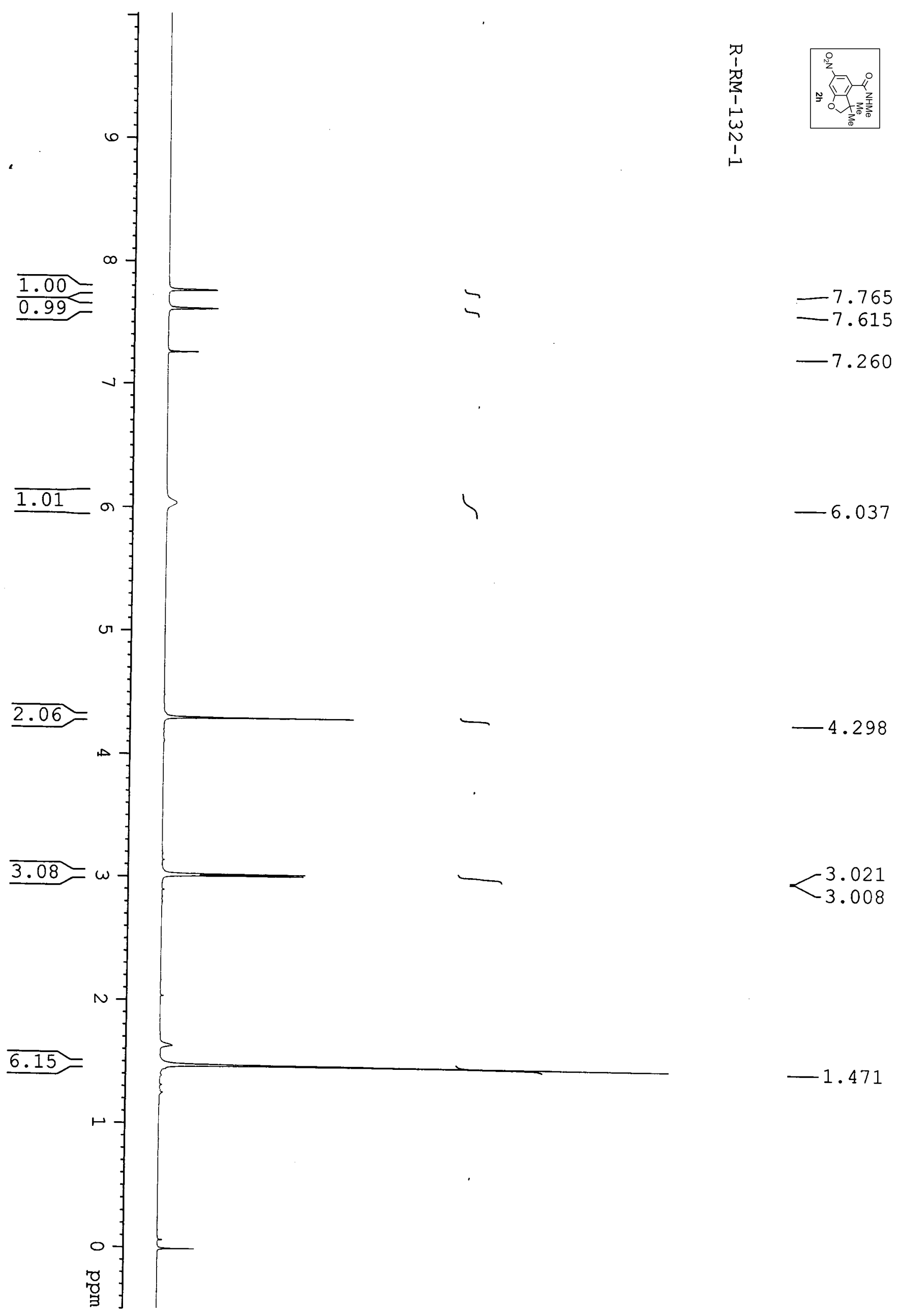




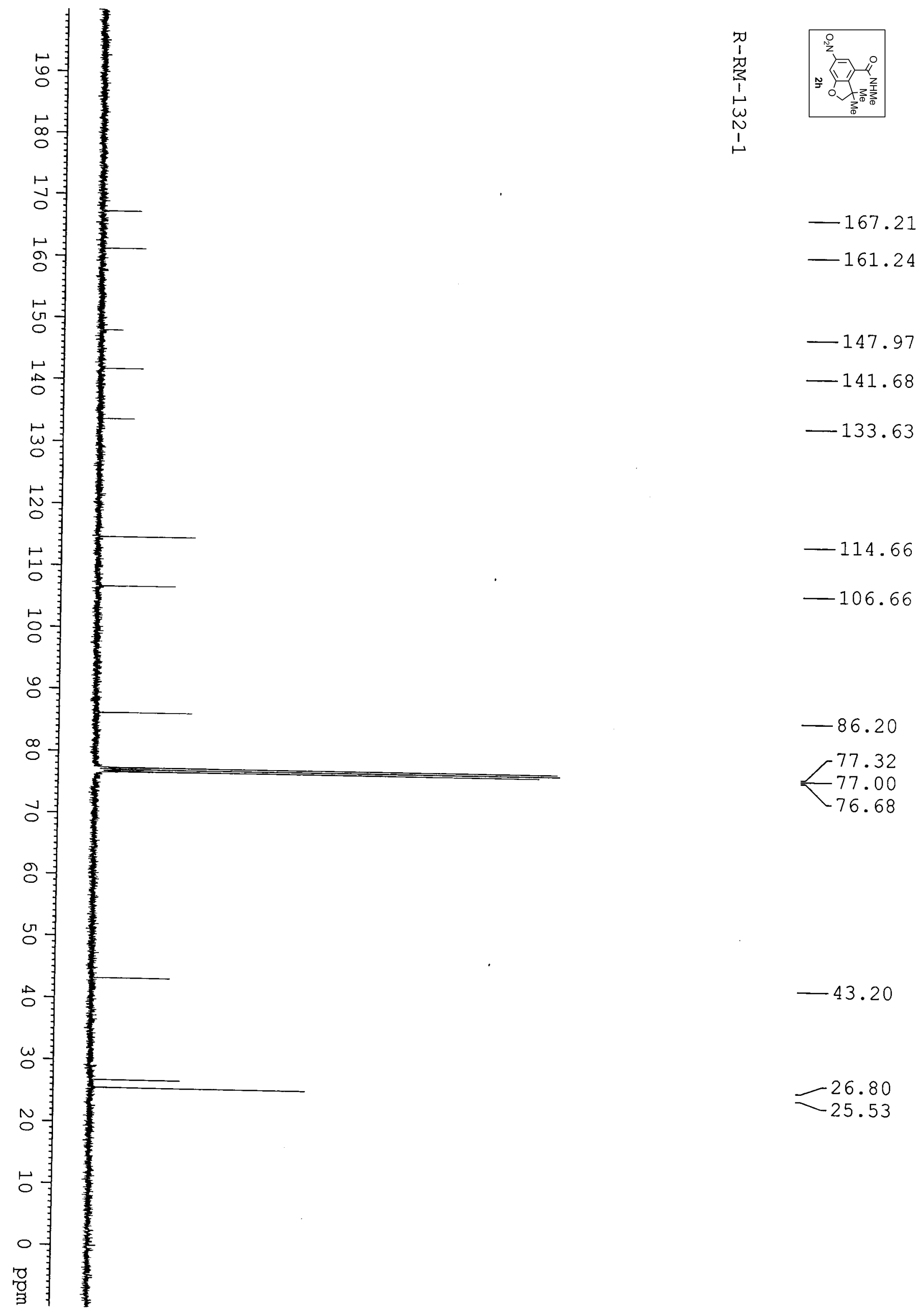




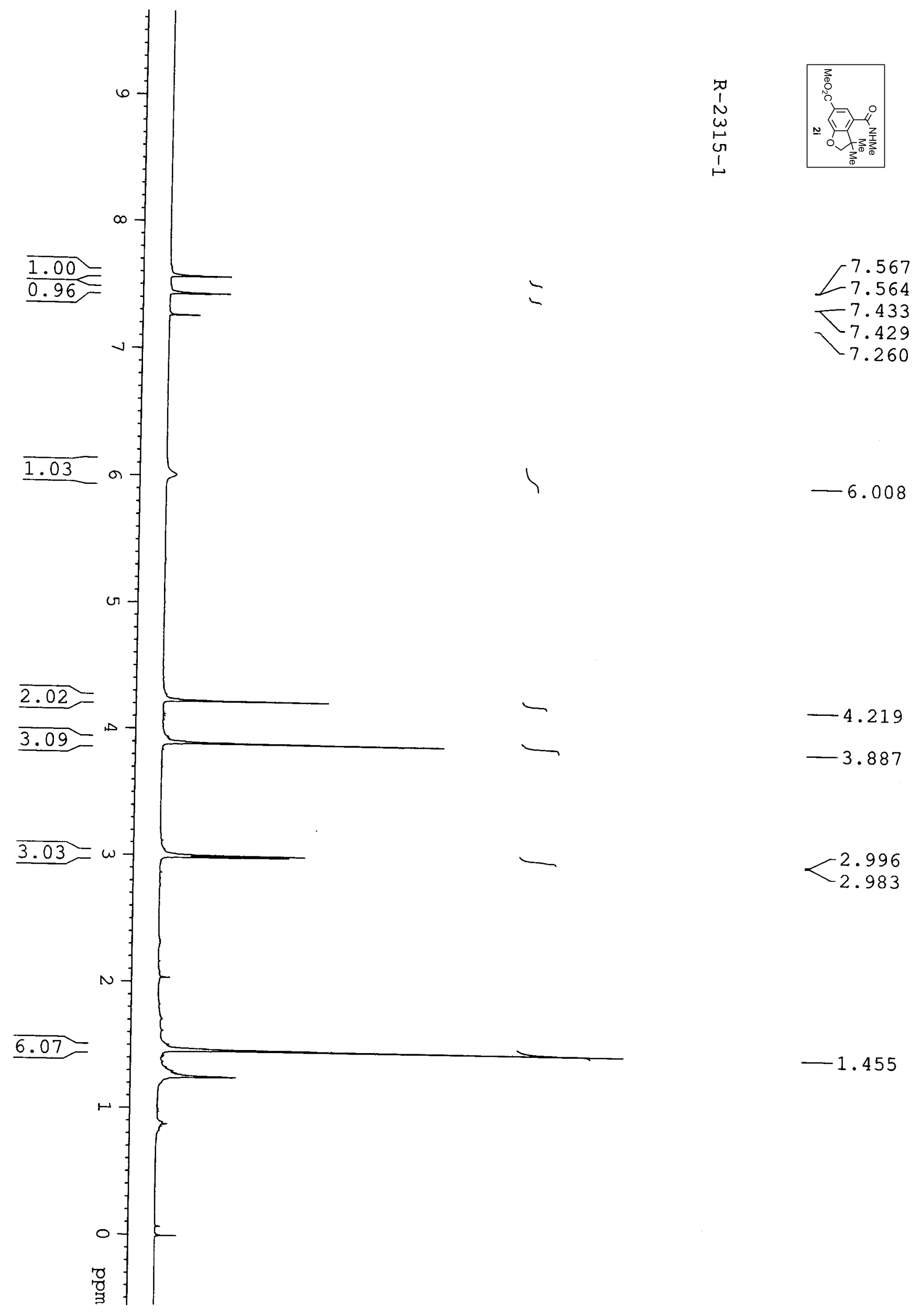




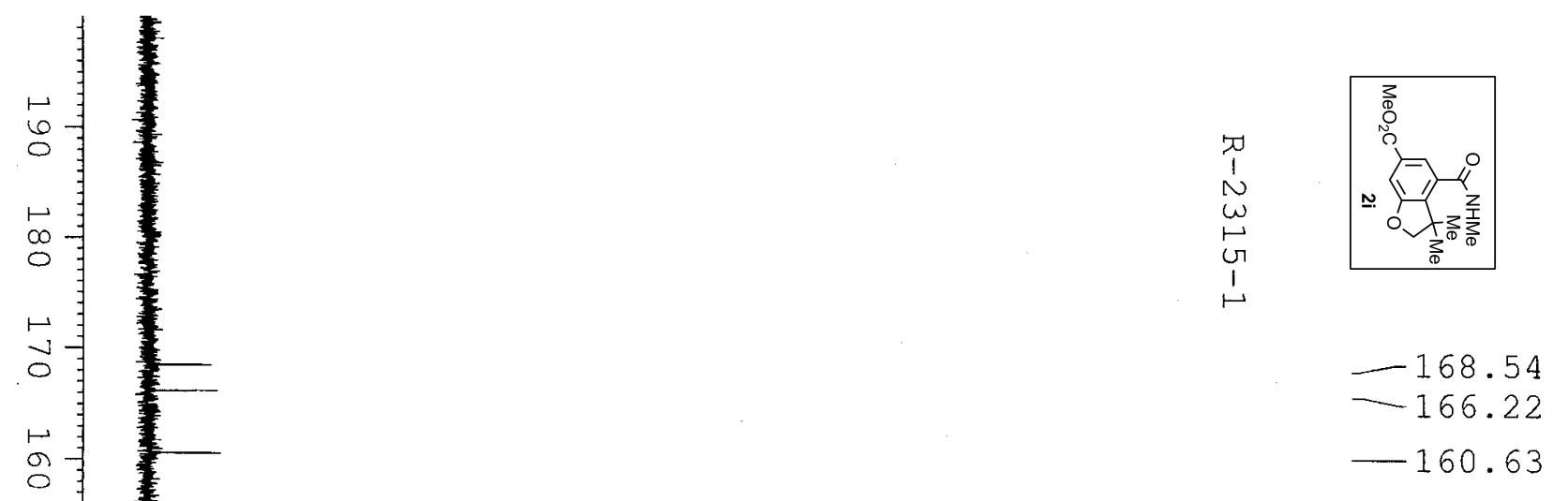

$-139.43$

$-133.32$

$-130.37$

$-120.76$

$-112.39$

䓃奉裹

5

웅

6

$\infty$

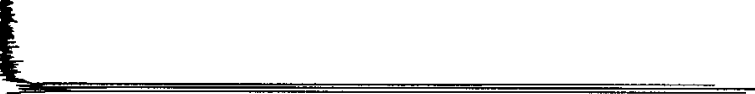

ป

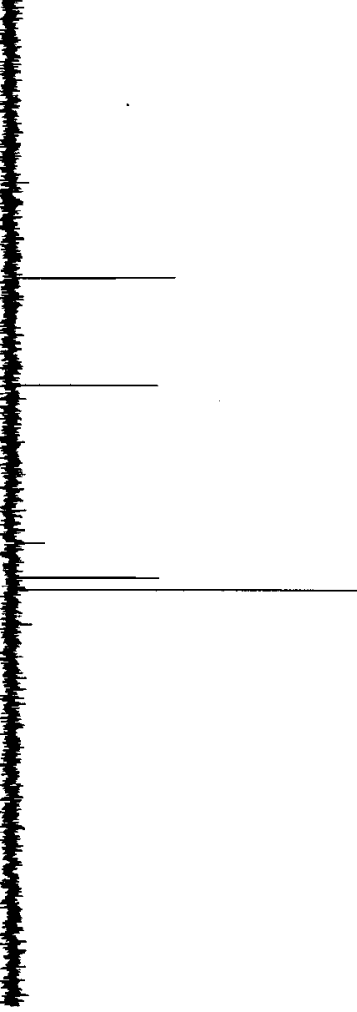

$-52.28$

$-43.11$

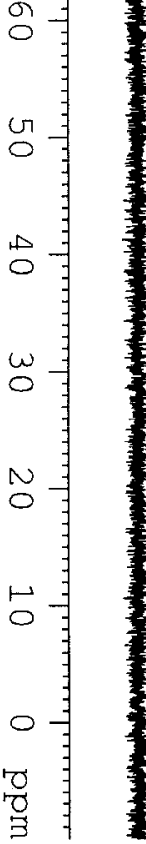

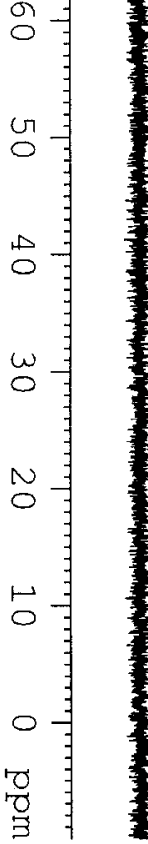

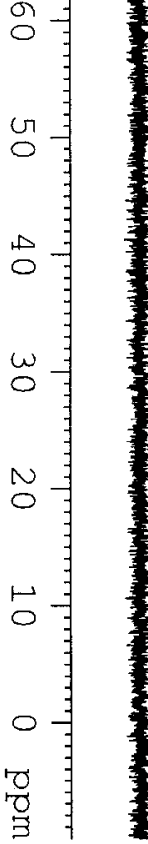

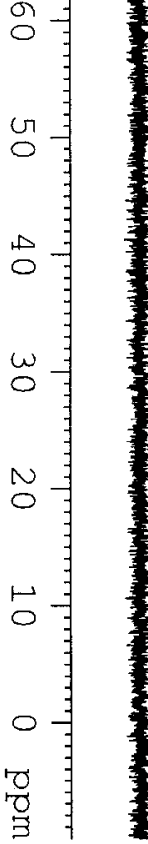

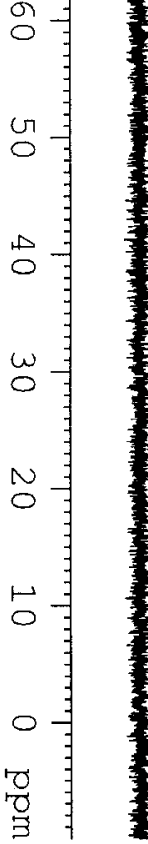

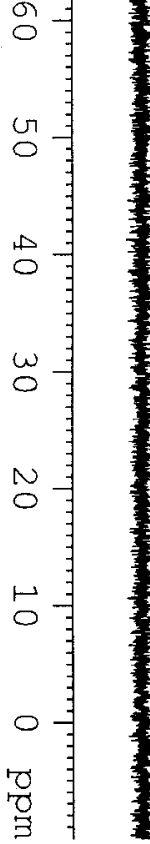

$-85.57$

$-77.32$

77.00

76.68

$-26.67$

$-25.63$ 

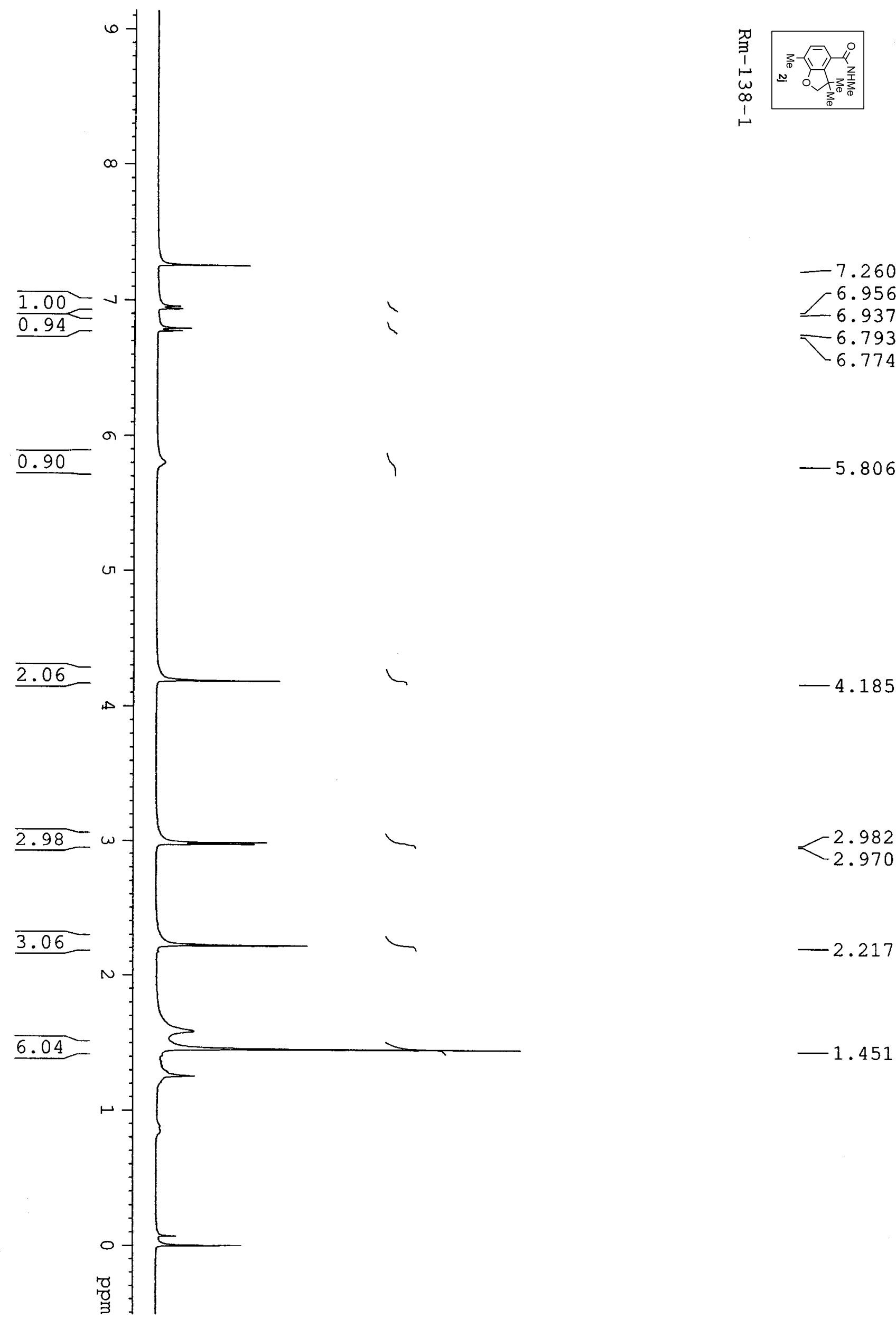

6.956

6.937

$-6.793$

6.774

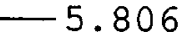

$-4.185$

$-2.982$

2.970

$-2.217$

$-1.451$ 


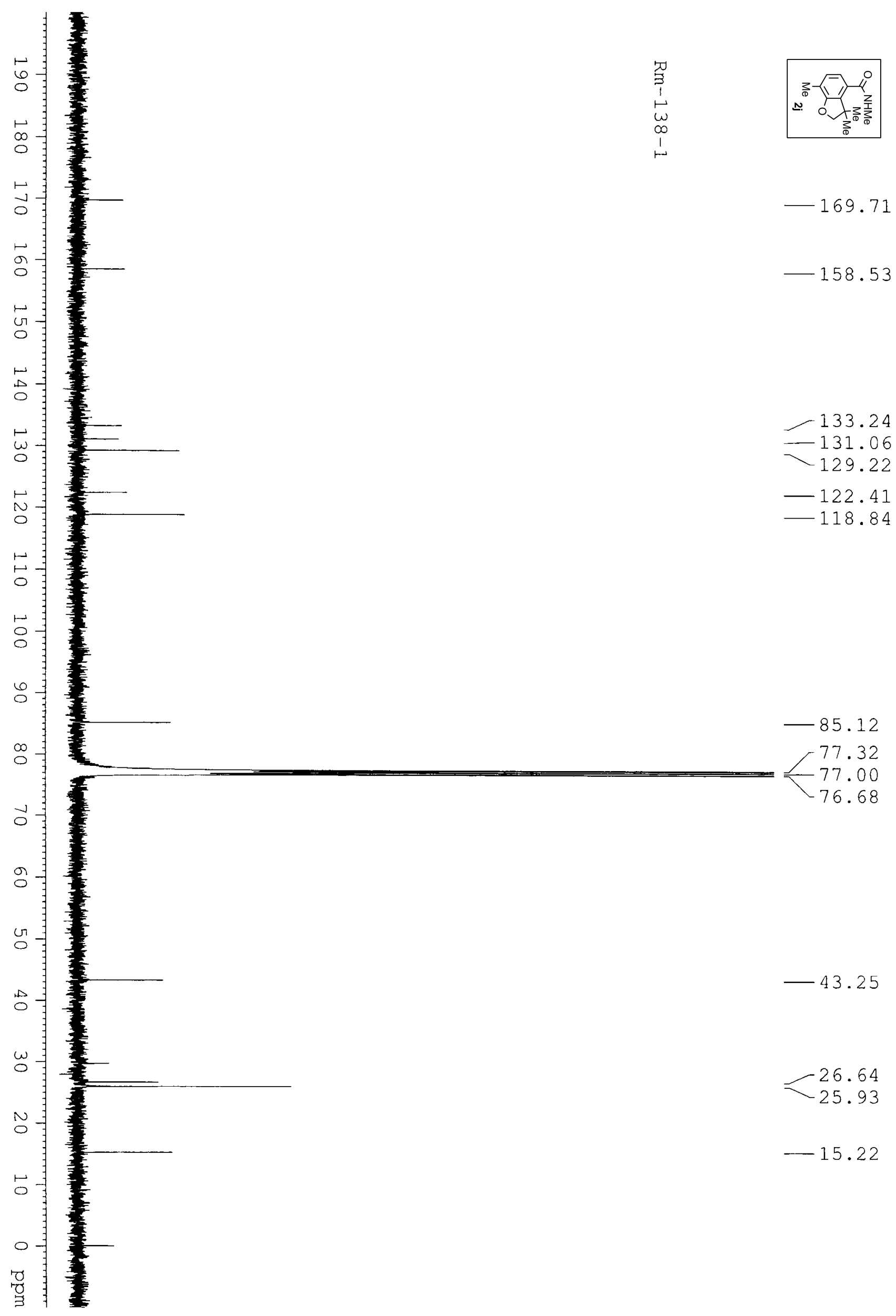




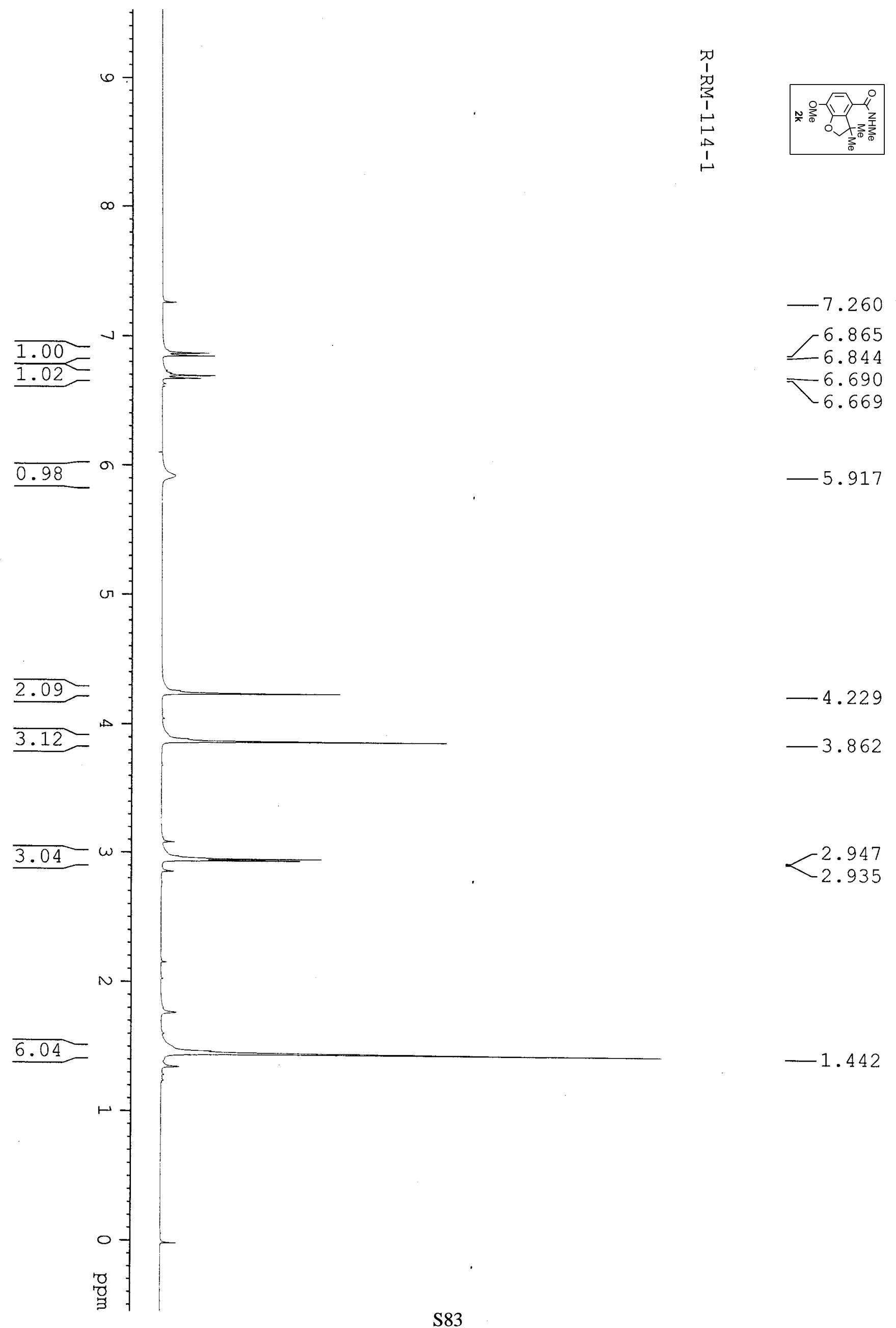



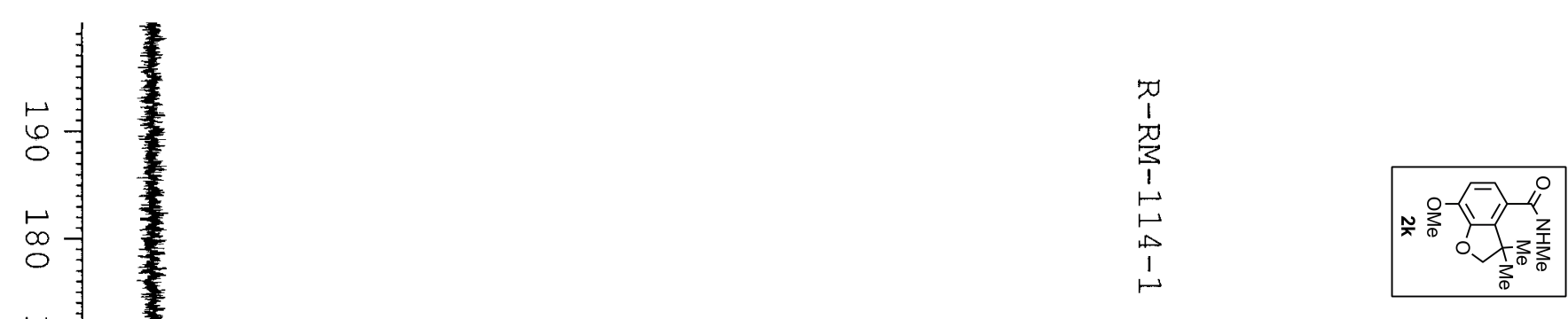

169.36

$-148.30$

$-146.15$

$-135.30$

$-125.93$

$-120.06$

$-110.21$

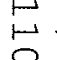

○

6

$\infty$

$-85.93$

77.32

$-77.00$

76.68

$\checkmark$

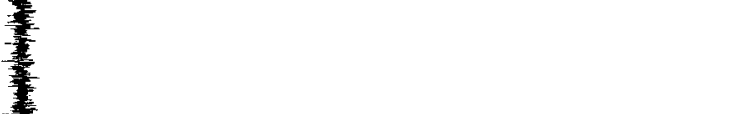

8

G

品奉罊

$\omega$

N

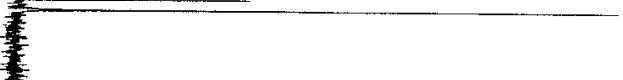

$-55.86$

$-43.70$

$-26.63$

$-25.75$ 


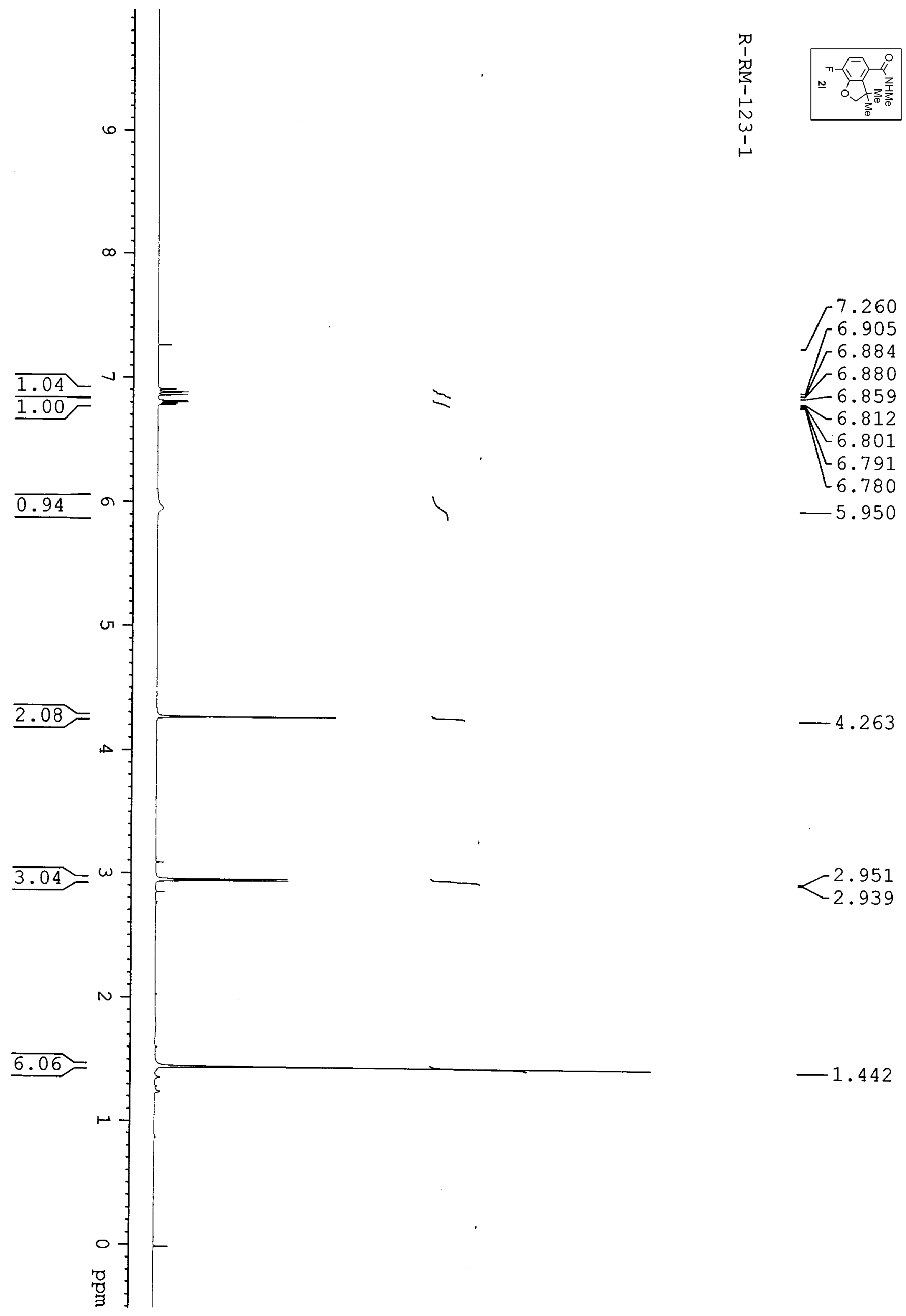




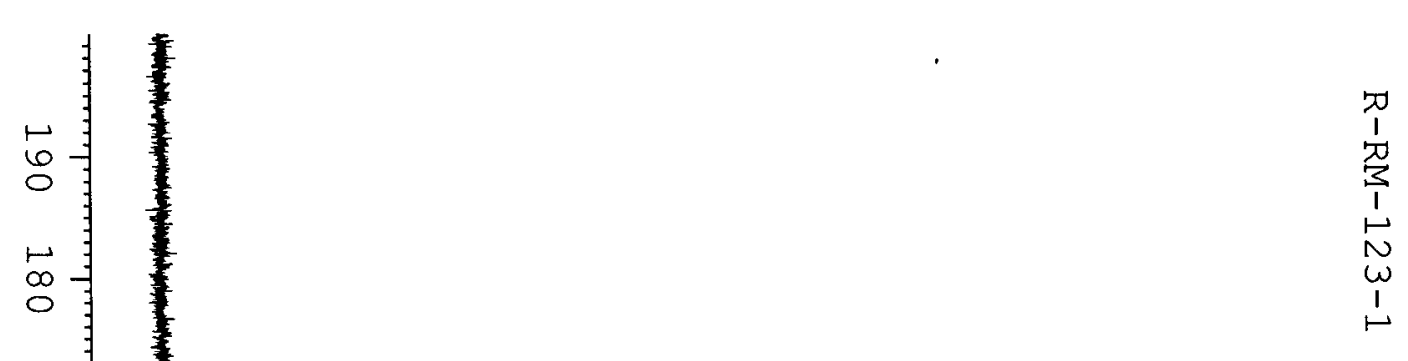

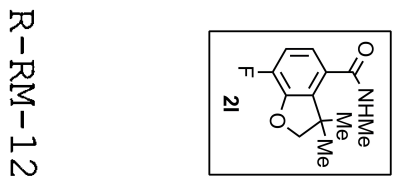

$-168.77$

。

ᄋ

빙

只

$\stackrel{8}{\circ}$

w

w

N

N

占

농

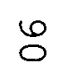

$\stackrel{\infty}{\circ}$

o

용

9

$\stackrel{\square}{\circ}$

$\omega$

N

$\circ$

○

$\circ$

莺

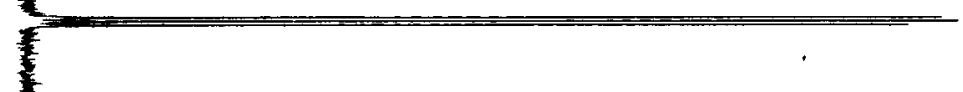

$-86.49$

77.32

77.00
-76.68

76.68

$-43.78$

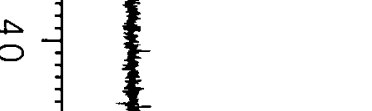

青

$\frac{1}{4}$

147.41

$-146.91$

146.81

137.92

129.28

$-129.25$

$-119.75$

114.99

-26.64
-25.63 

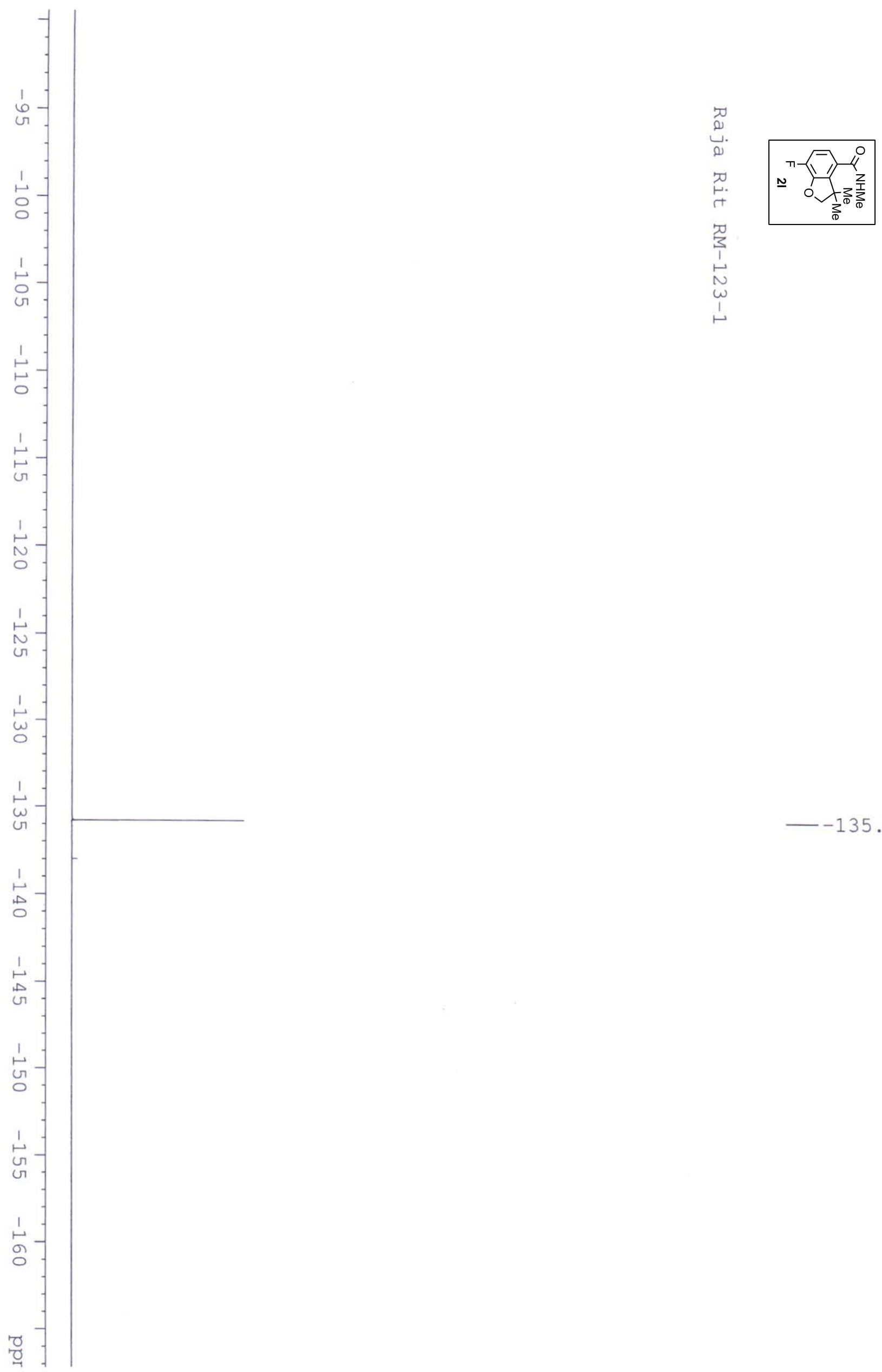

$--135.79$ 


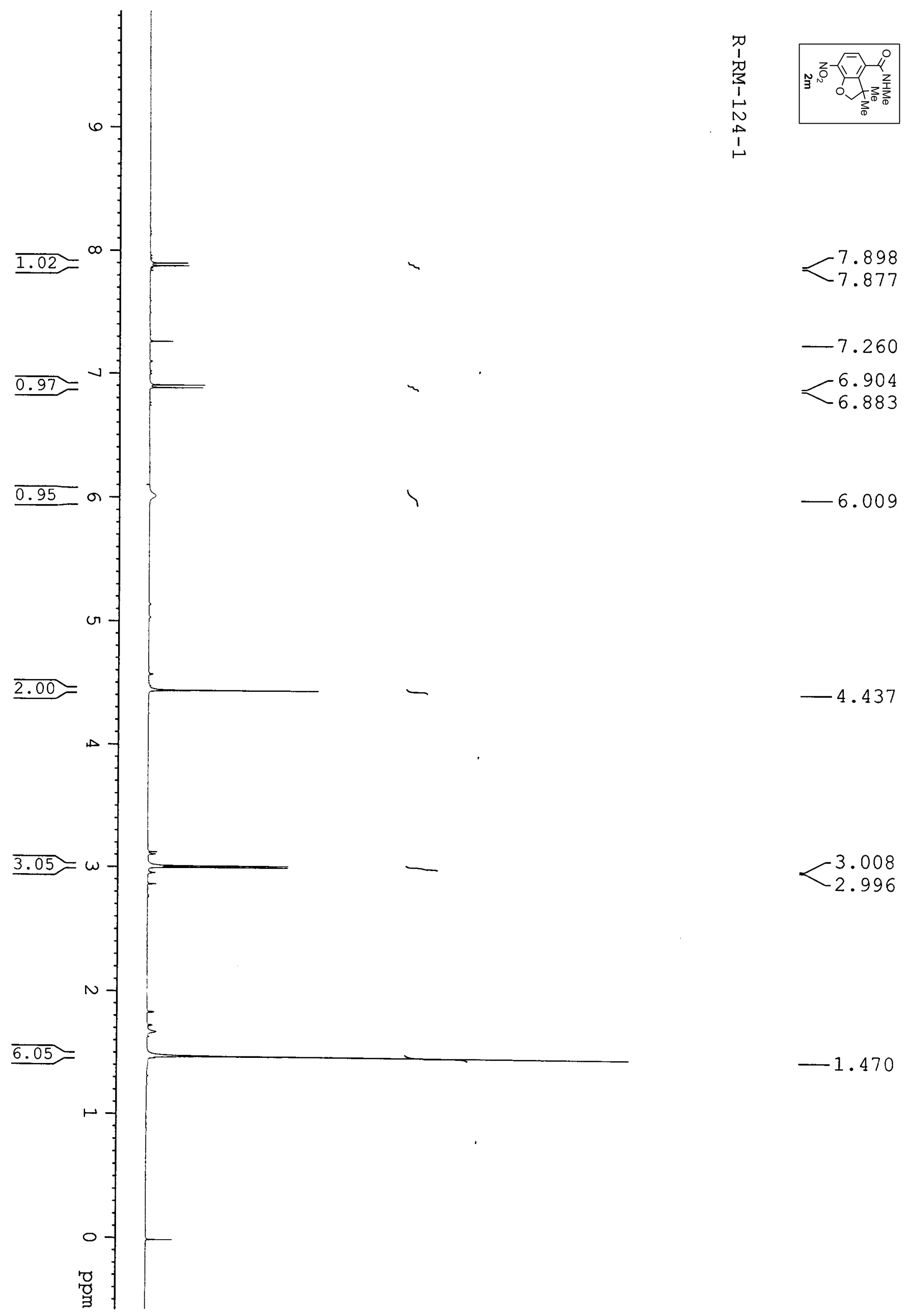



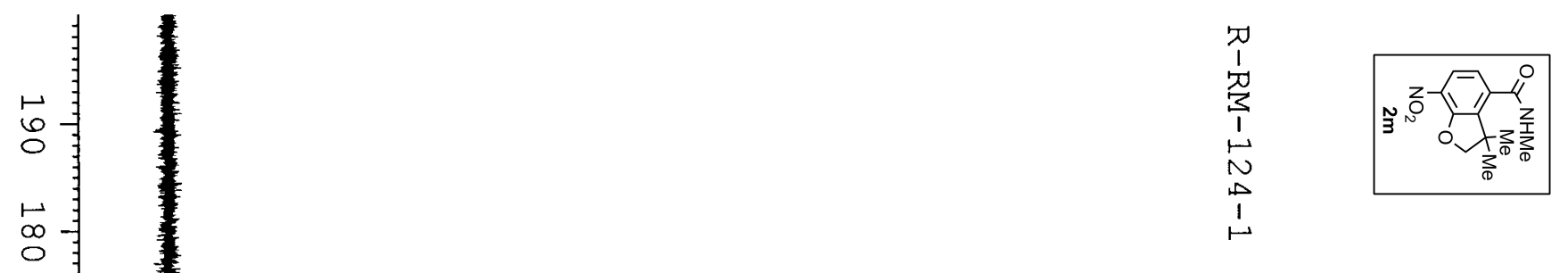

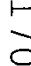

$-167.57$

$$
\text { 㫐 }
$$

s.

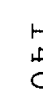

落

$\stackrel{\leftrightarrow}{\circ}$

它

N

•

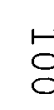

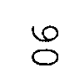

$\infty$$$
\text { s }
$$

o

u

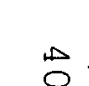$$
5
$$

$\omega$

N

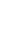

5

$\circ$

竞

'0 


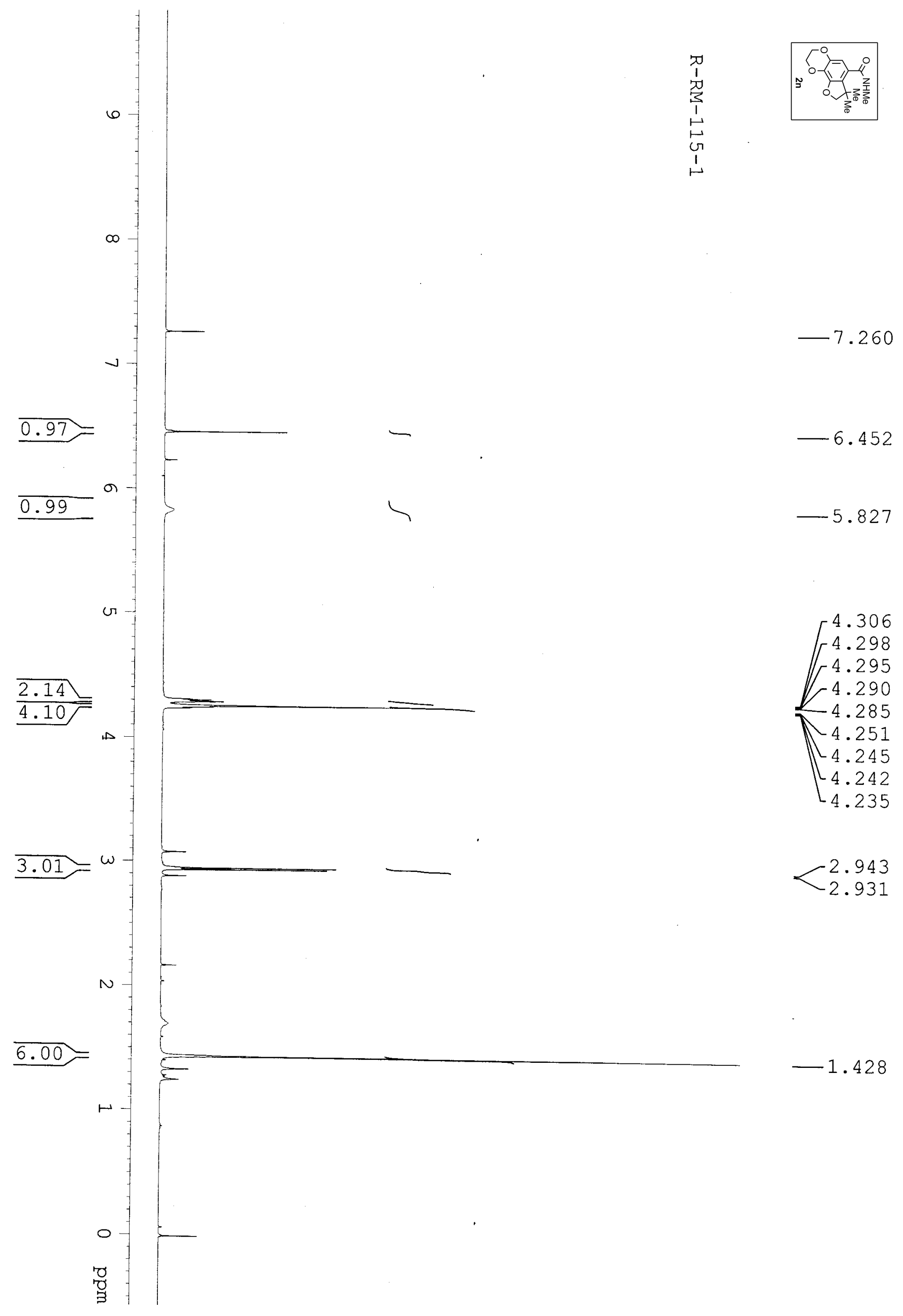




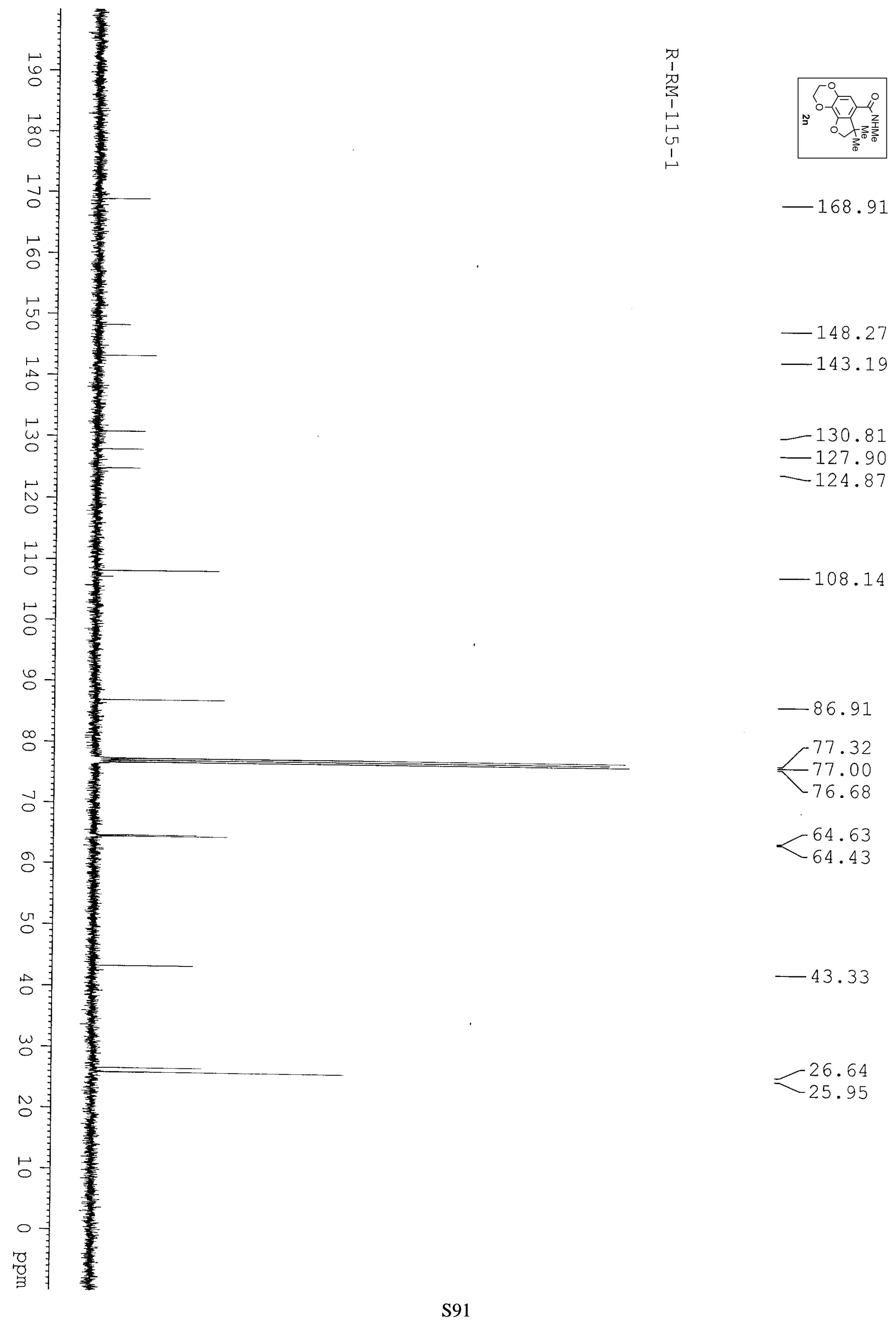




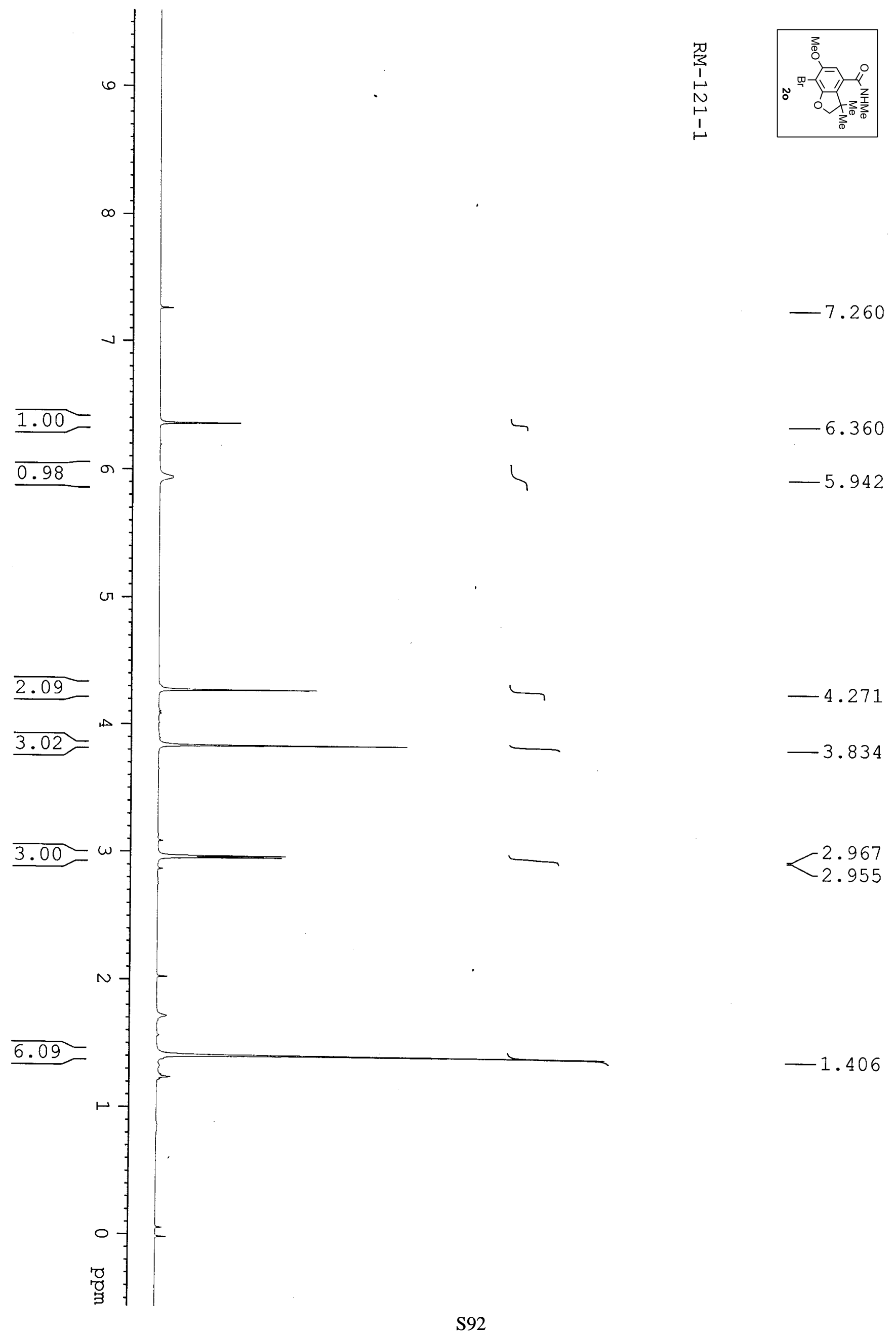




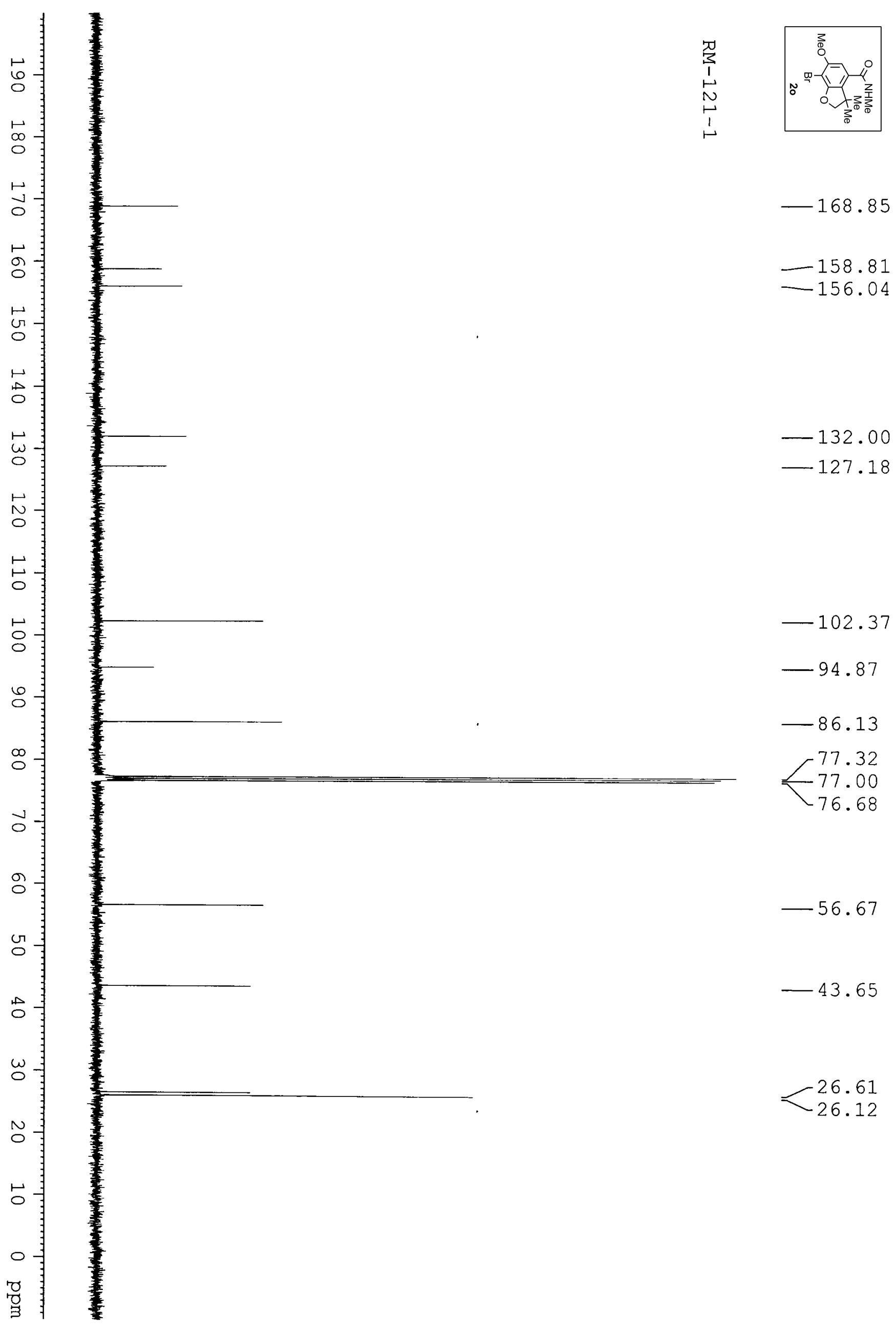




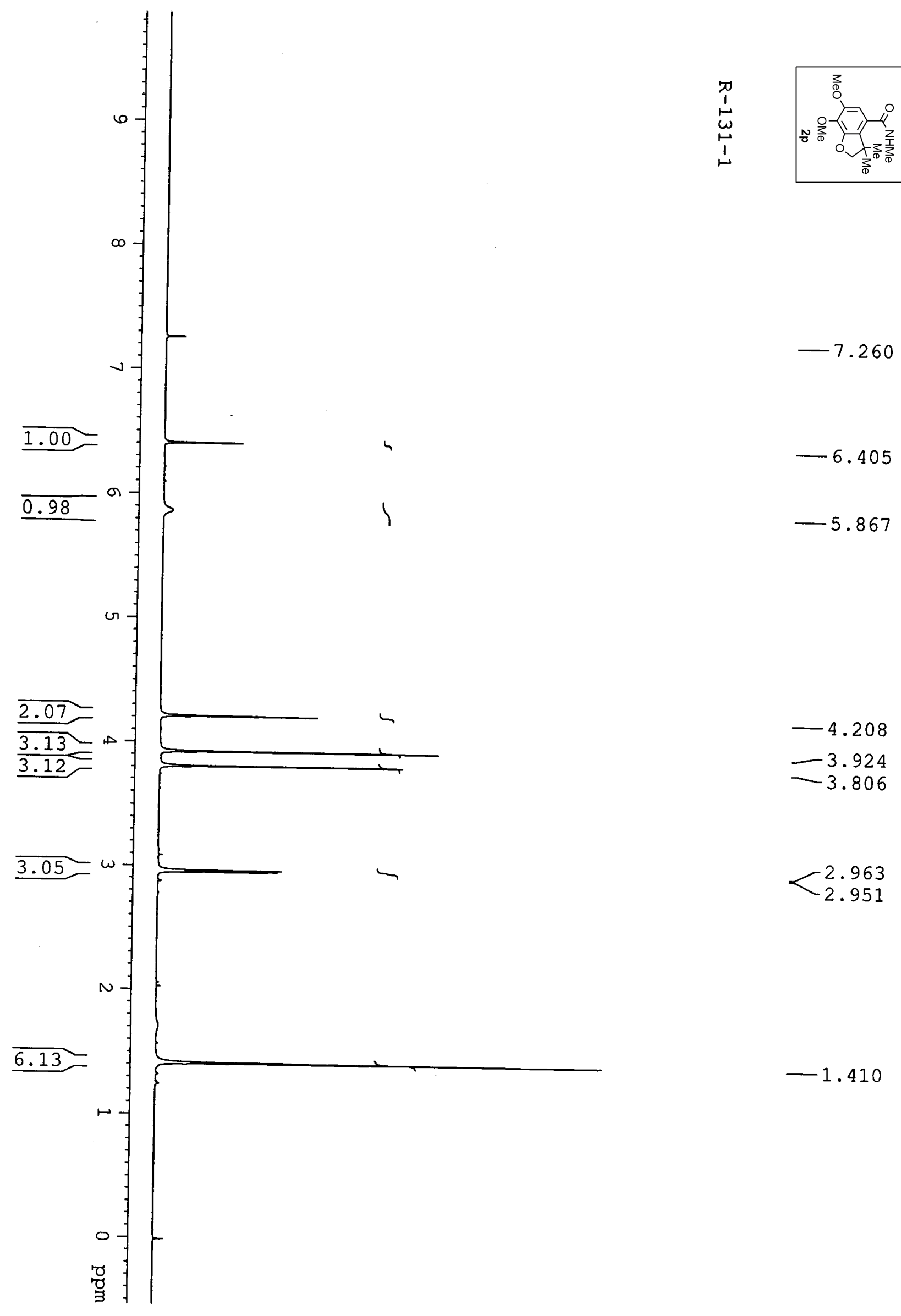




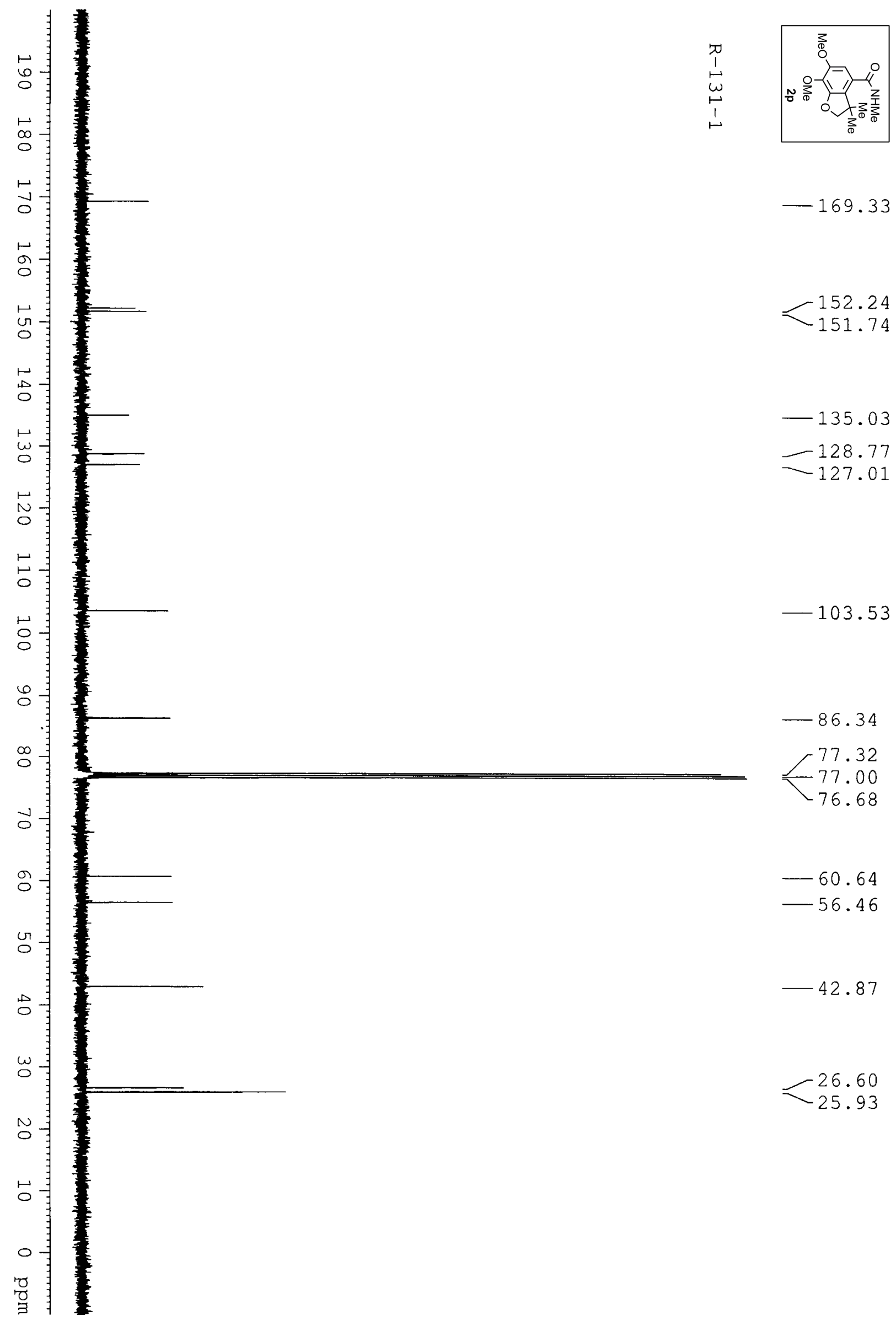



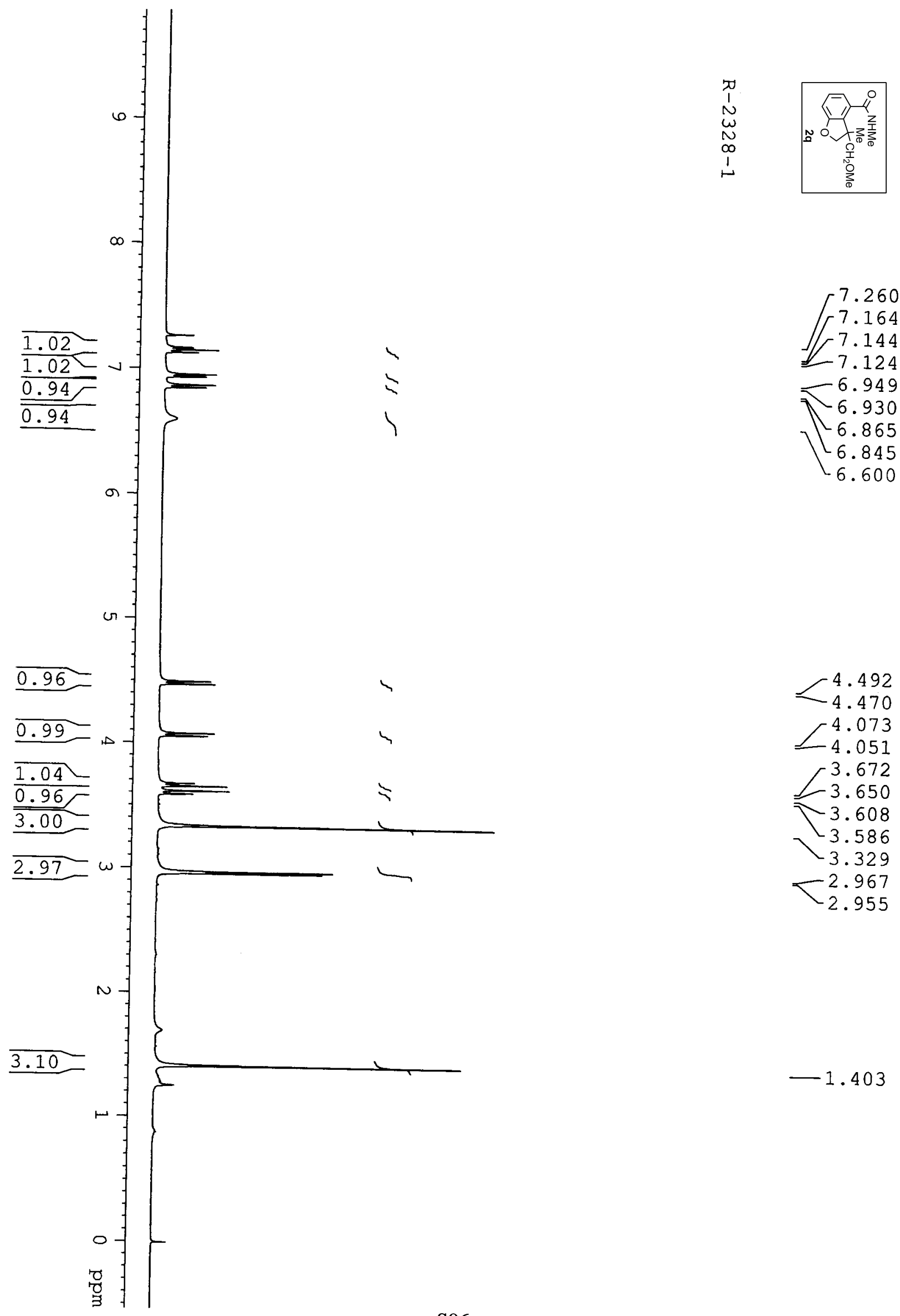

7.164
7.144

$-7.124$

$-6.949$

$-6.930$

$-6.865$

6.845

6.600

4.492

4.470
-4.073

4.073

4.051

3.672

$-3.650$

$-3.608$

3.586

3.329

$-2.967$

2.955 

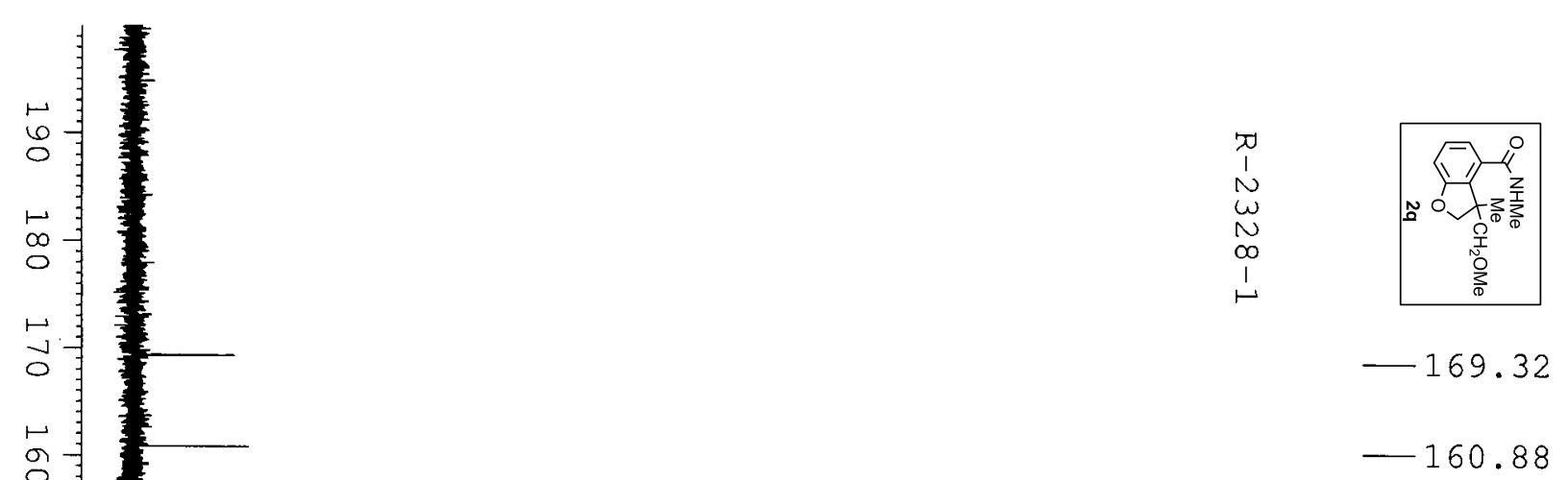

$-134.46$

$-129.56$

$\begin{array}{r}128.75 \\ \hline\end{array}$

$-119.66$

$-111.87$

占

뭉

6

$\infty$

ठิ

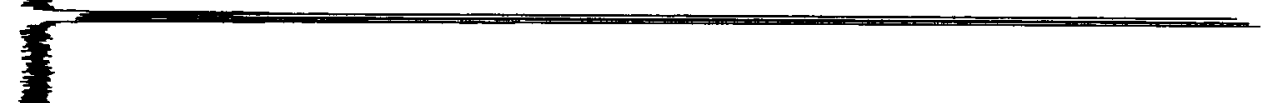

81.07

77.48

$-77.32$

$-77.00$

76.68

$-59.19$

$-47.63$

G

$\stackrel{\circ}{\circ}$

$\omega$

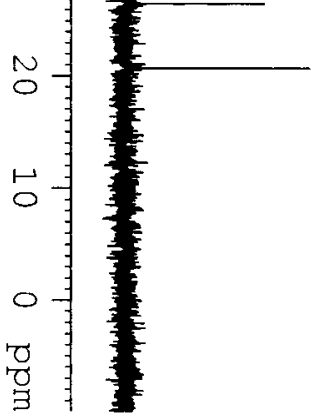

$-26.55$

$-20.70$ 

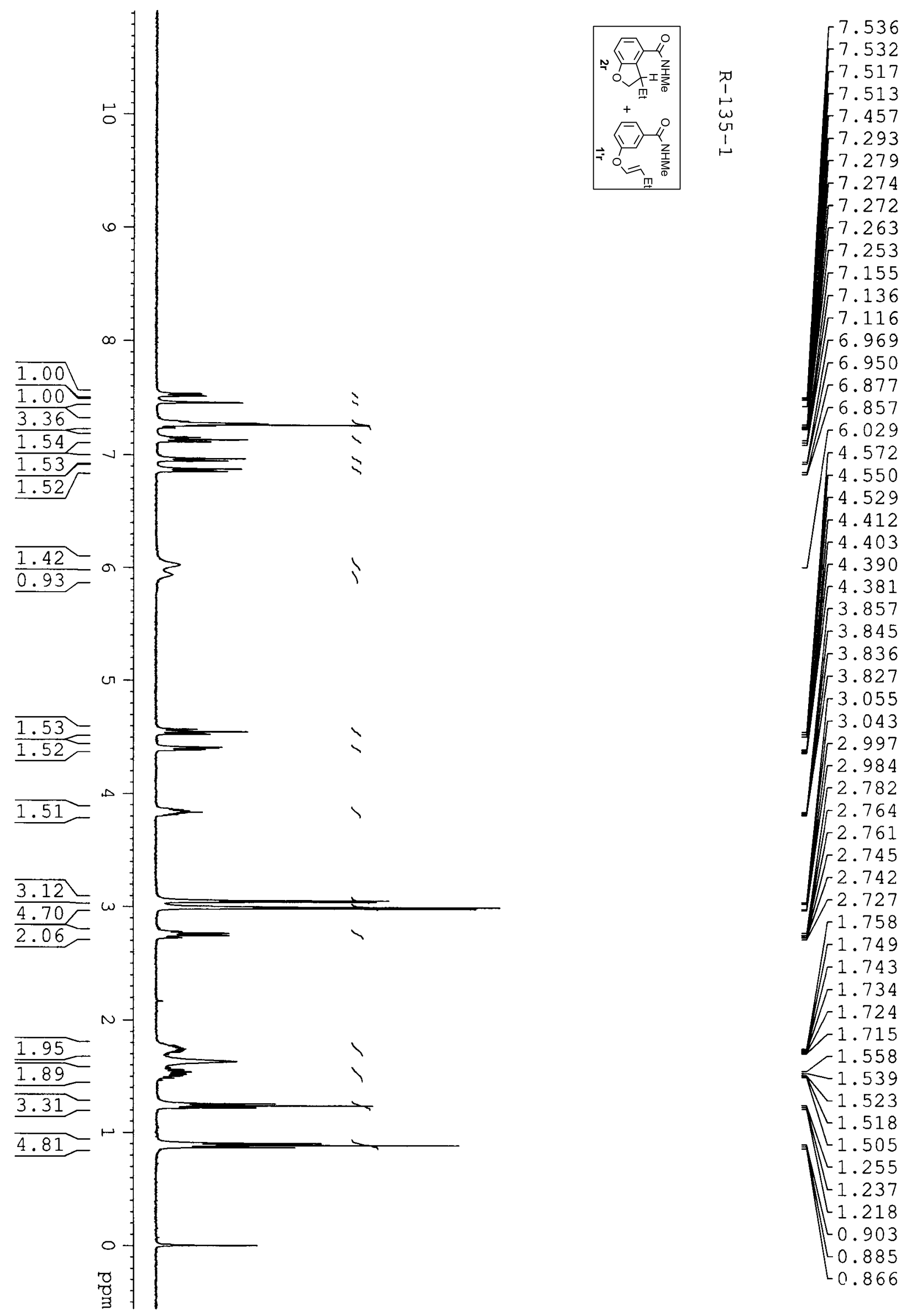

7.272

7.263

$-7.253$

$-7.155$

7.136

7.116

6.969

6.950

6.877

$\left[\begin{array}{c}6.857 \\ 6.029\end{array}\right.$

$=\left[\begin{array}{l}6.029 \\ 4.572\end{array}\right.$

$=\left[\begin{array}{l}4.572 \\ 4.550\end{array}\right.$

4.529

4.412

$\left[\begin{array}{l}4.403 \\ 4.390\end{array}\right.$

4.381

3.857

3.845

$-3.836$

$-3.827$

3.055

3.043

2.997

2.984

2.782

2.764

2.761

2.745

2.742

$-2.727$

1.758

1.749

1.743

$\Gamma^{1.734}$

1.724

$-1.715$

$-1.558$

1.539

1.523

1.518

1.505
1.255

1.255

1.237

1.218

0.903

0.885

0.866 


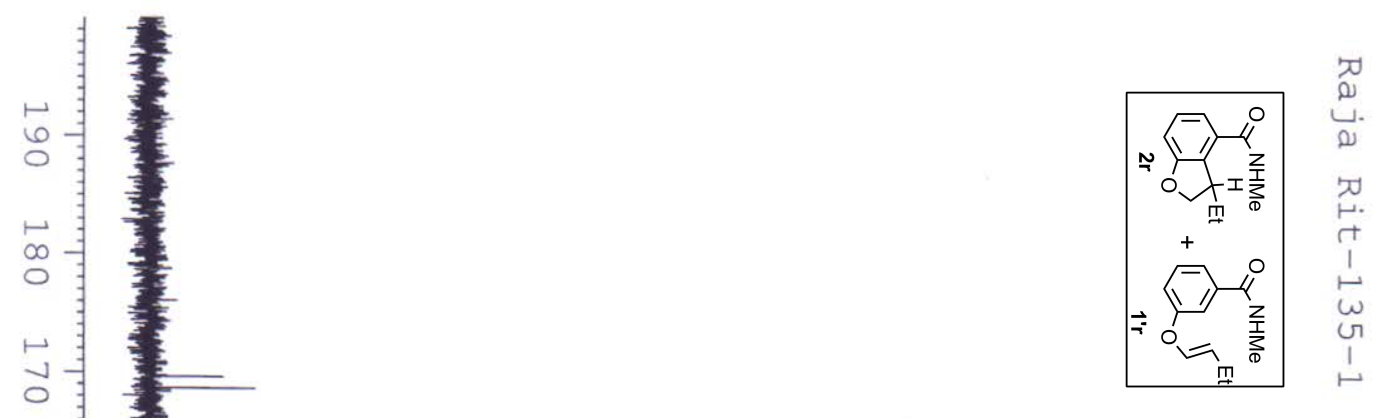

169.62
-168.63
-160.75
-156.08

$-142.23$

132.40

130.79

130.60

$-128.30$

$-125.05$

$-123.59$

$-122.64$

$-120.91$

118.21

113.36

111.84

○응

6

$\stackrel{\infty}{\circ}$

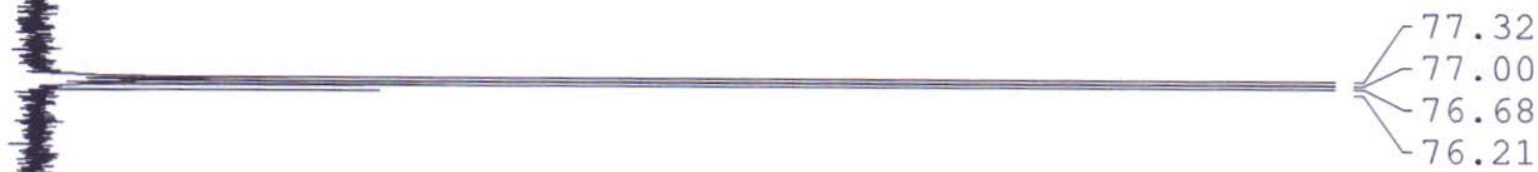

$\checkmark$

옹

ज

$\triangle$

$-42.81$



N.

O

5

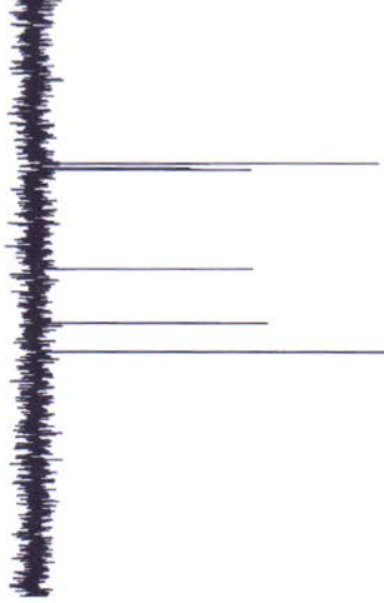

$-18.14$

$-13.47$

$-11.02$ 


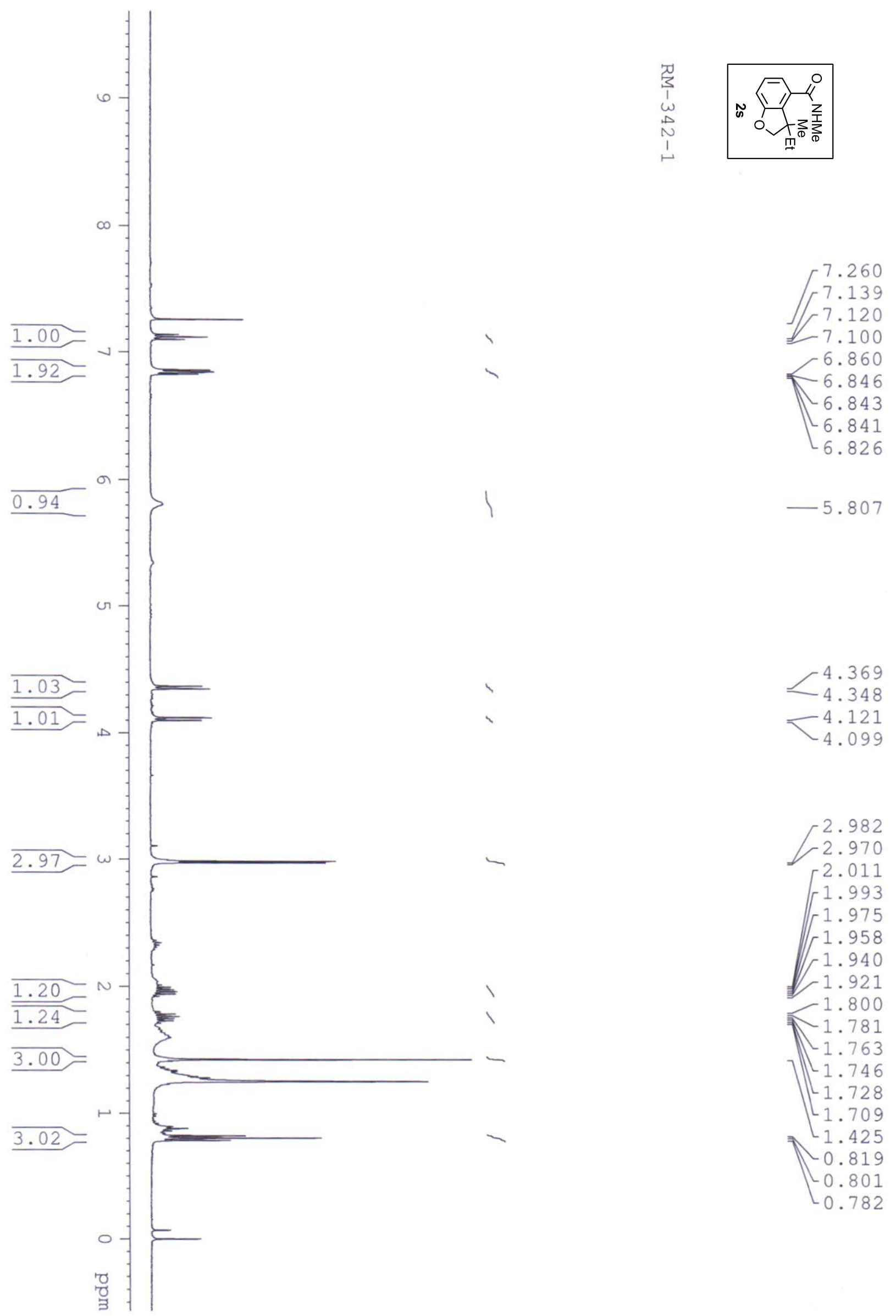




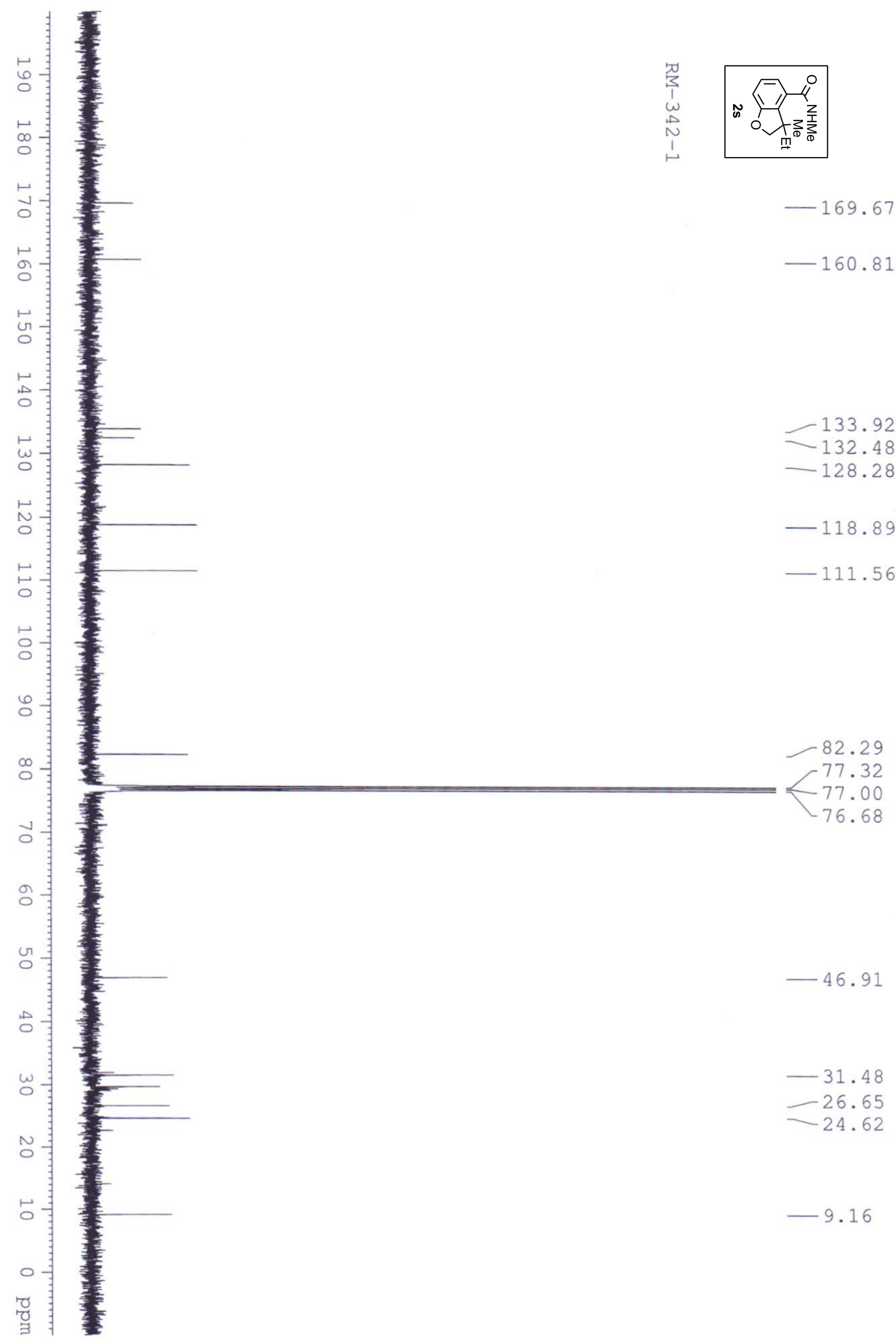




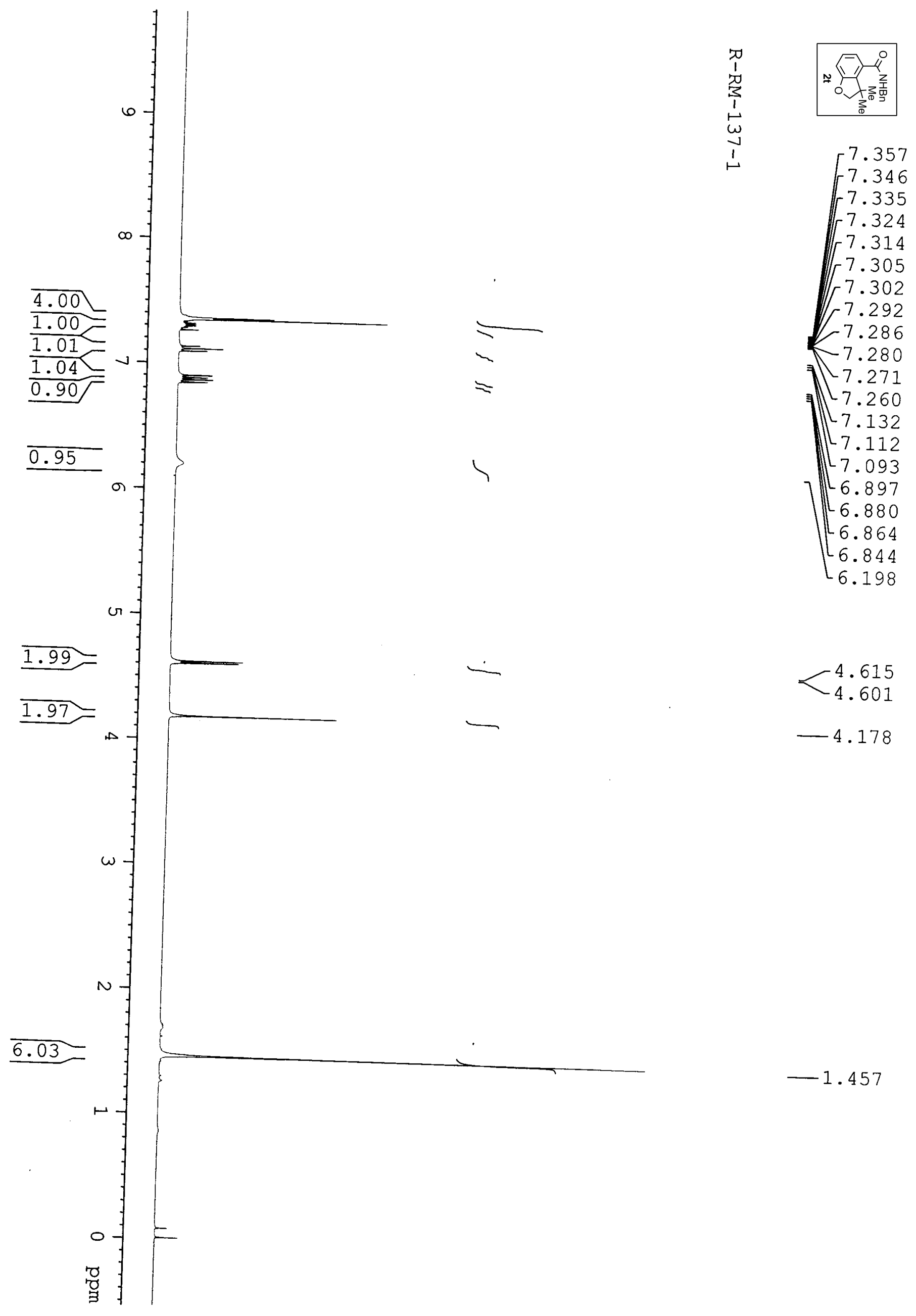




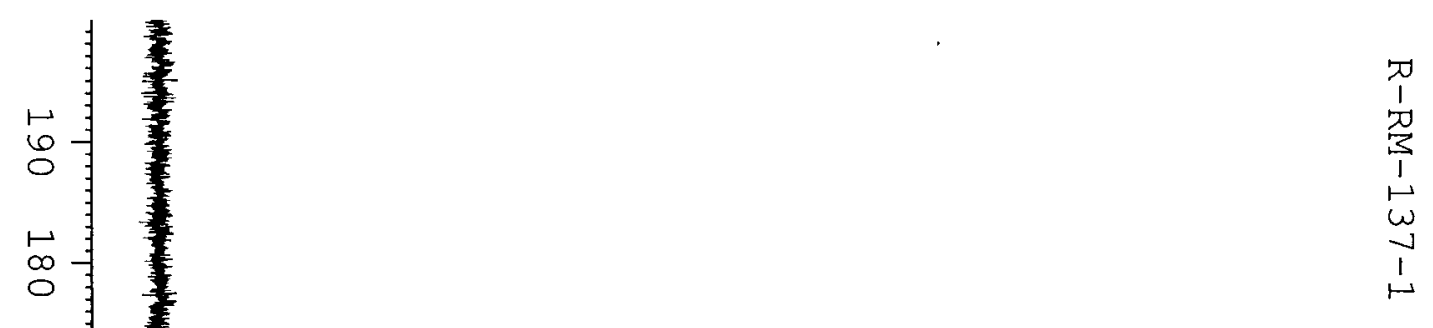

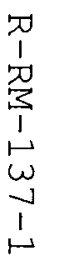

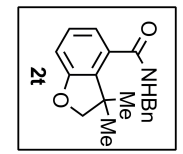

$-168.64$

$-160.34$

o

जั

$\stackrel{\leftrightarrow}{\circ}$

$\omega$

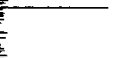

N
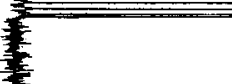

$\circ$

卢

$\stackrel{+}{\circ}$

6

$\infty$

o

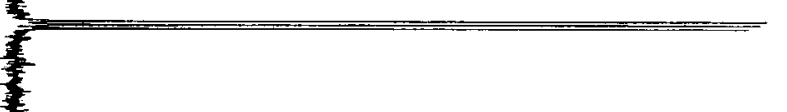

$-85.24$

77.32

$-77.00$

$-76.68$

134.10

$-133.30$

$-128.75$

$-128.24$

$-127.82$

127.60

118.93

$-111.94$

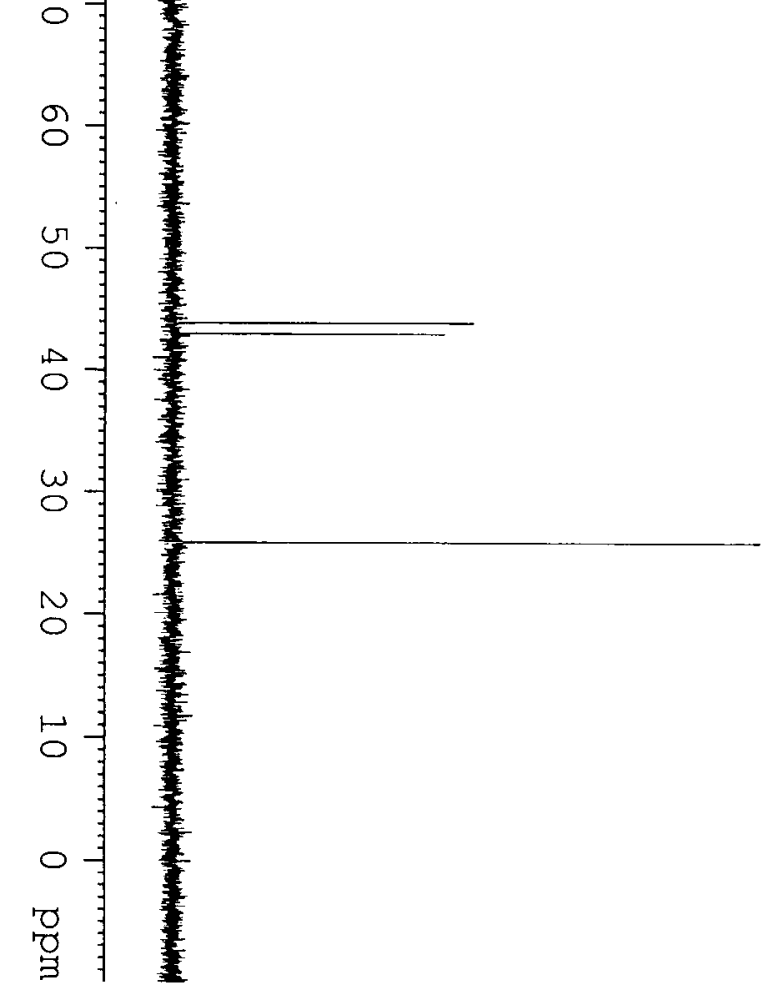

$-43.88$

42.97

$-25.86$ 

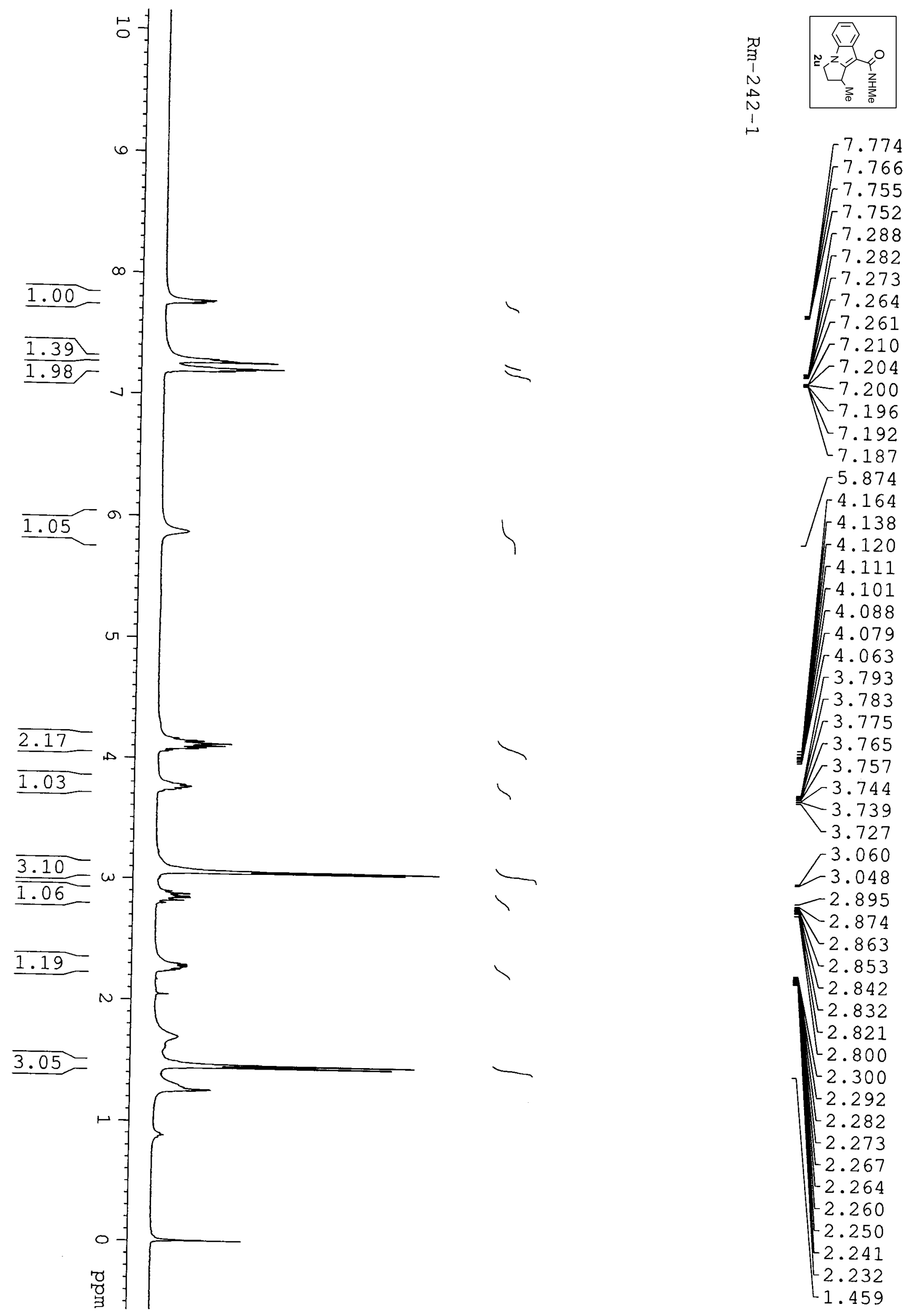

7.774

7.766

7.755

7.752

7.288

7.282

7.273

7.264

7.261

7.210

$-7.204$

$-7.200$

$-7.196$

7.192

7.187

5.874

$\int \begin{aligned} & 4.164 \\ & 4.138 \\ & 4.120\end{aligned}$

4.111

$-4.101$

$-4.088$

4.079

4.063

3.793

3.783

3.775

$r^{3.765}$

3.757

$-3.744$

$-3.739$

3.727

3.060

3.048

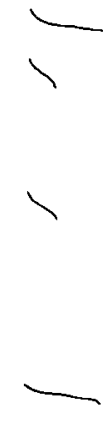

2.895

$-2.874$

2.863

$-2.853$

2.842

2.832

2.821

2.800

2.300

$-2.292$

2.282

2.273

2.267

$-2.264$

2.260

2.250

2.241

2.232

ᄂ.459 


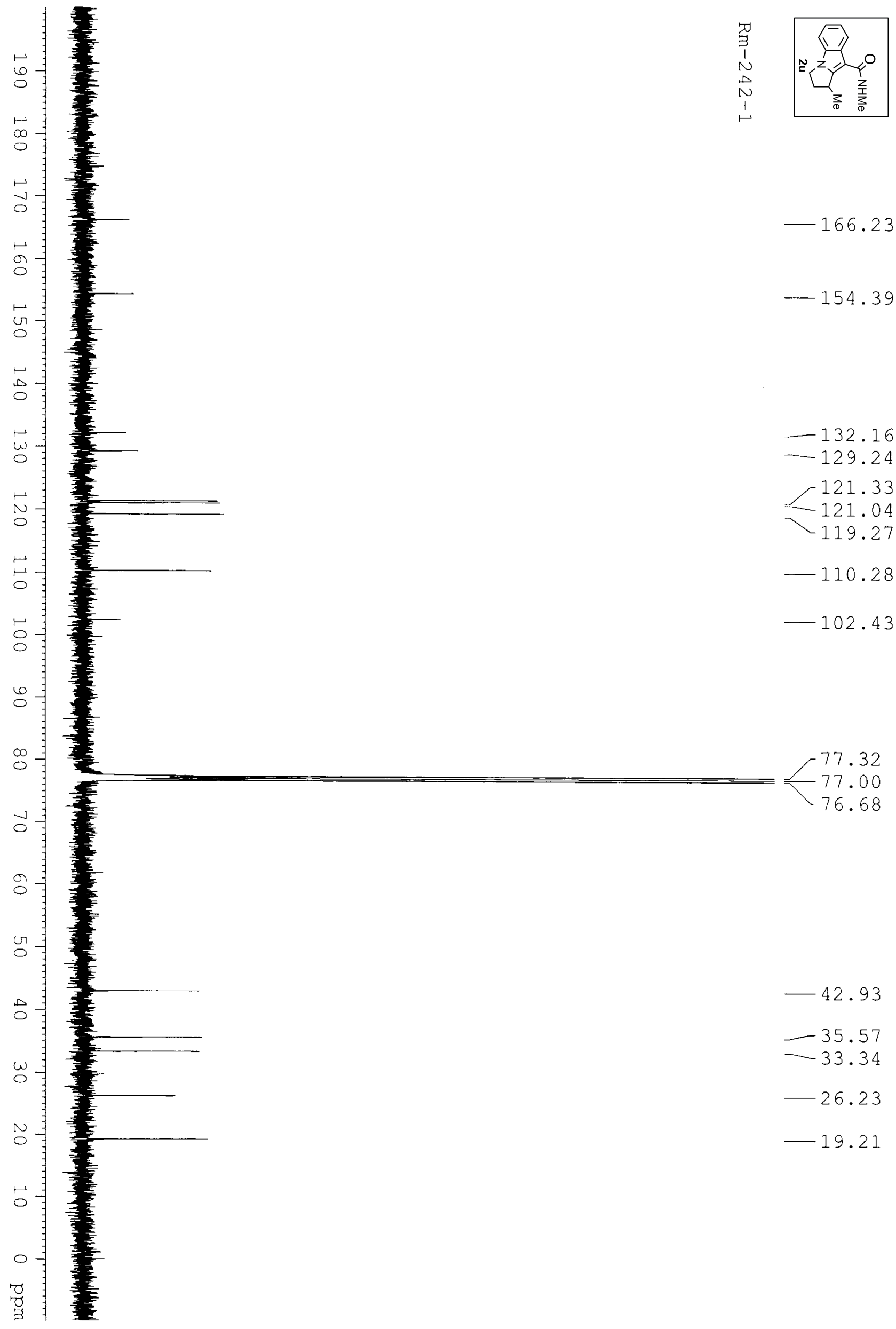



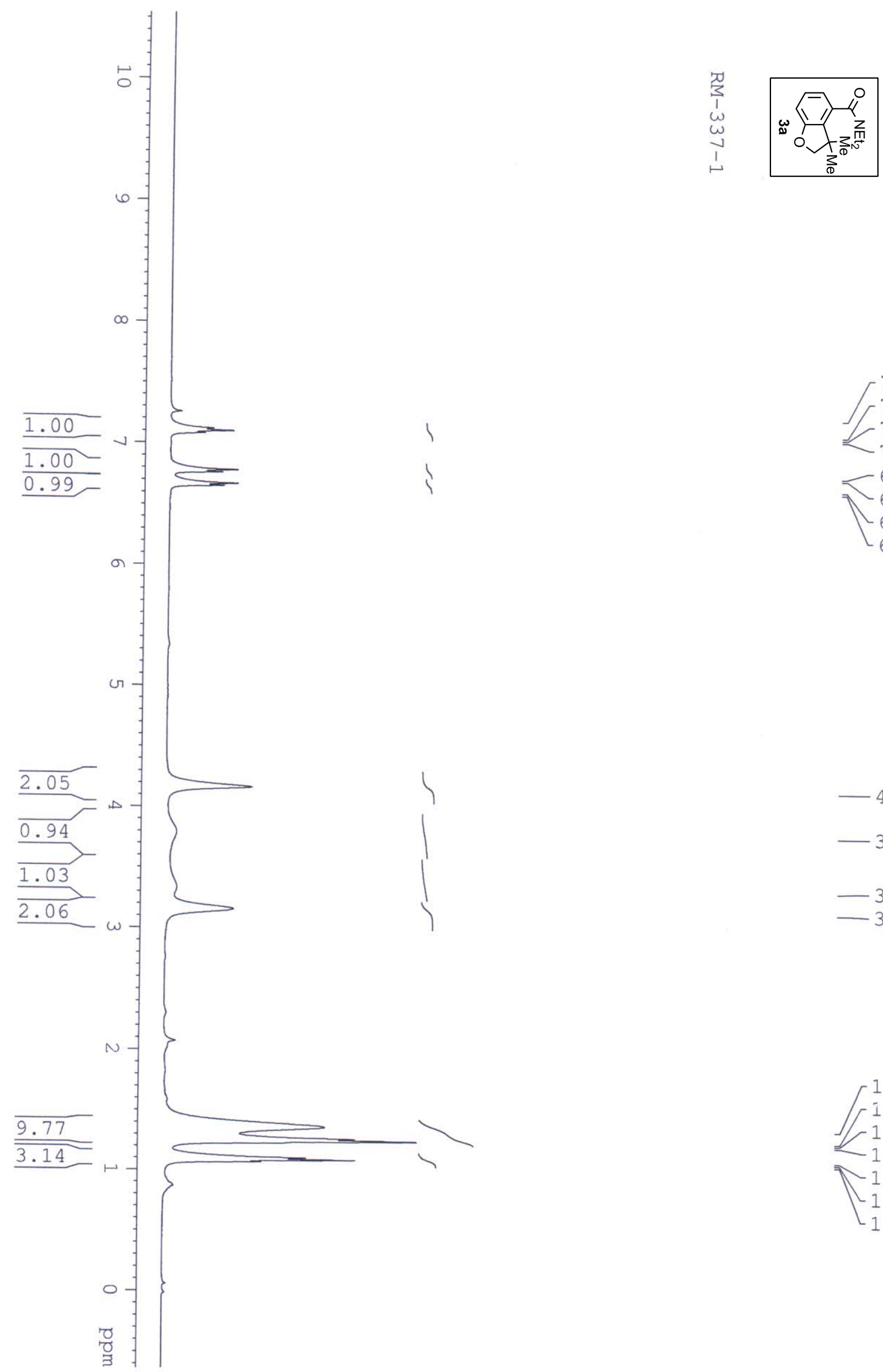

7.260

7.123

$-7.104$

$-7.085$

$-6.786$

$-6.766$

$-6.674$

6.656

$-4.169$

$-3.795$

$-3.337$

$-3.160$

1.367
1.265
1.248
1.231
1.104
1.088
1.071 

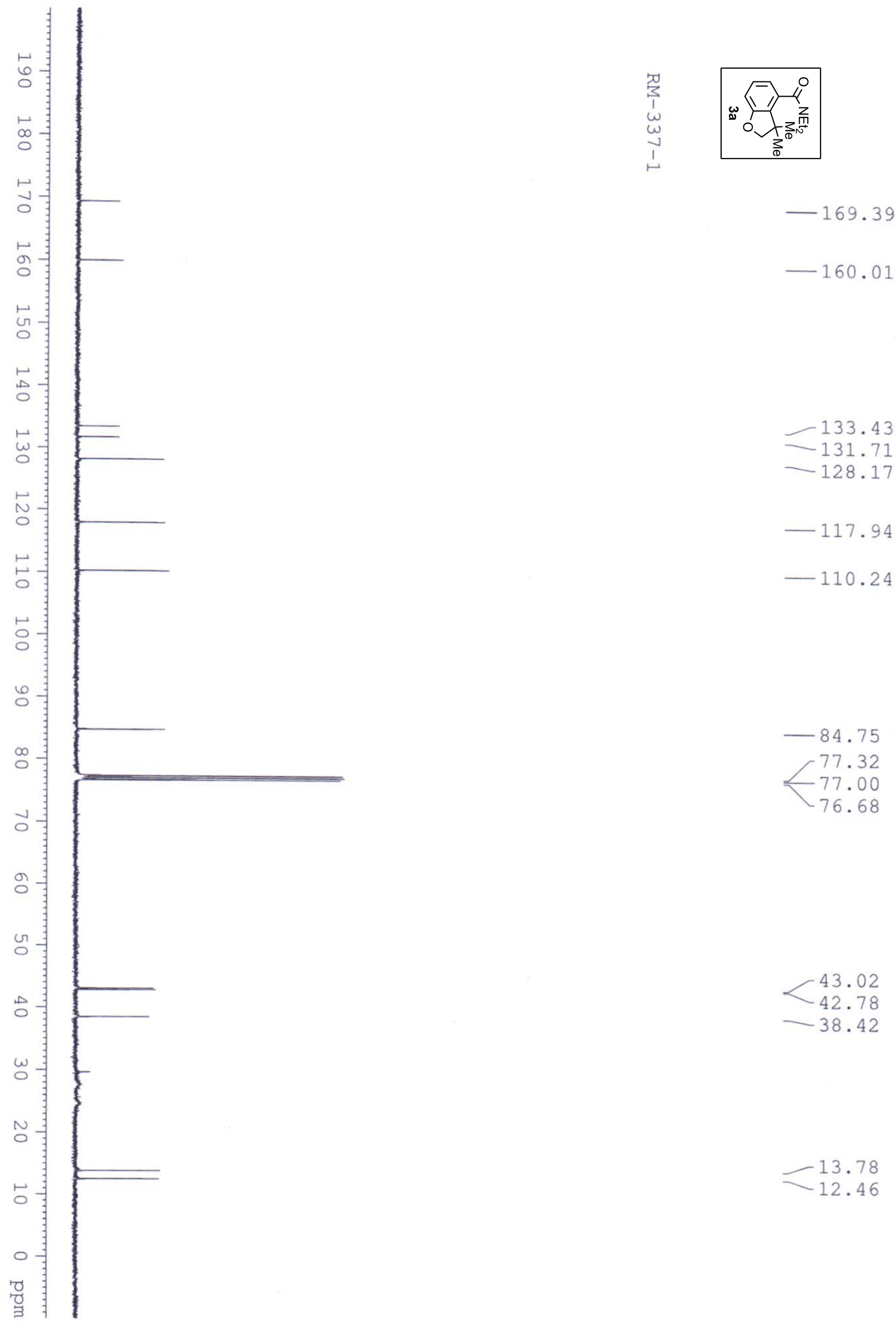

$-160.01$

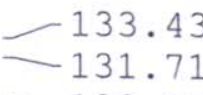

$-128.17$

$-117.94$

$-110.24$

$-84.75$

77.32

$-77.00$

76.68

$-43.02$

42.78

$-38.42$

$-13.78$

$-12.46$ 


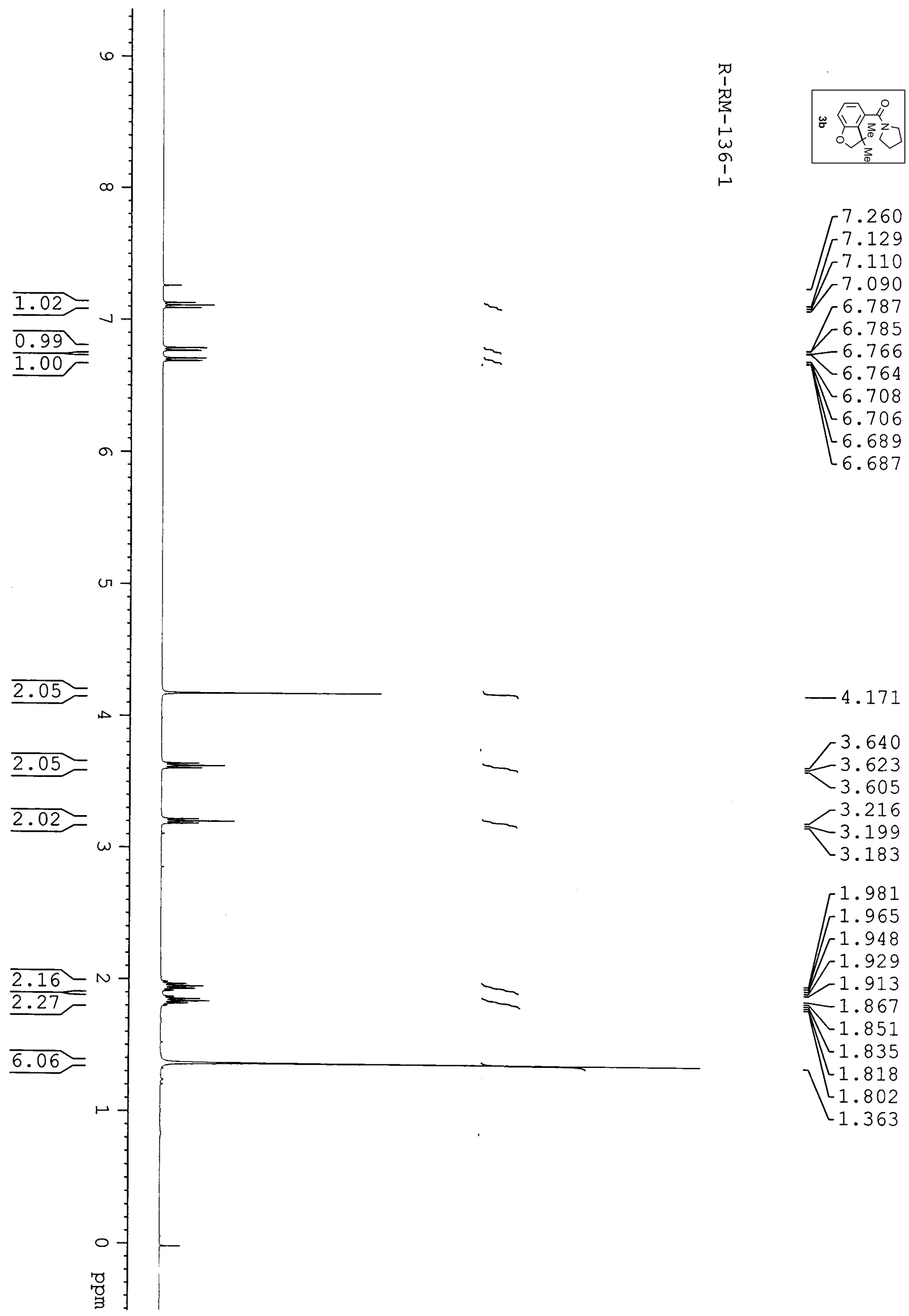



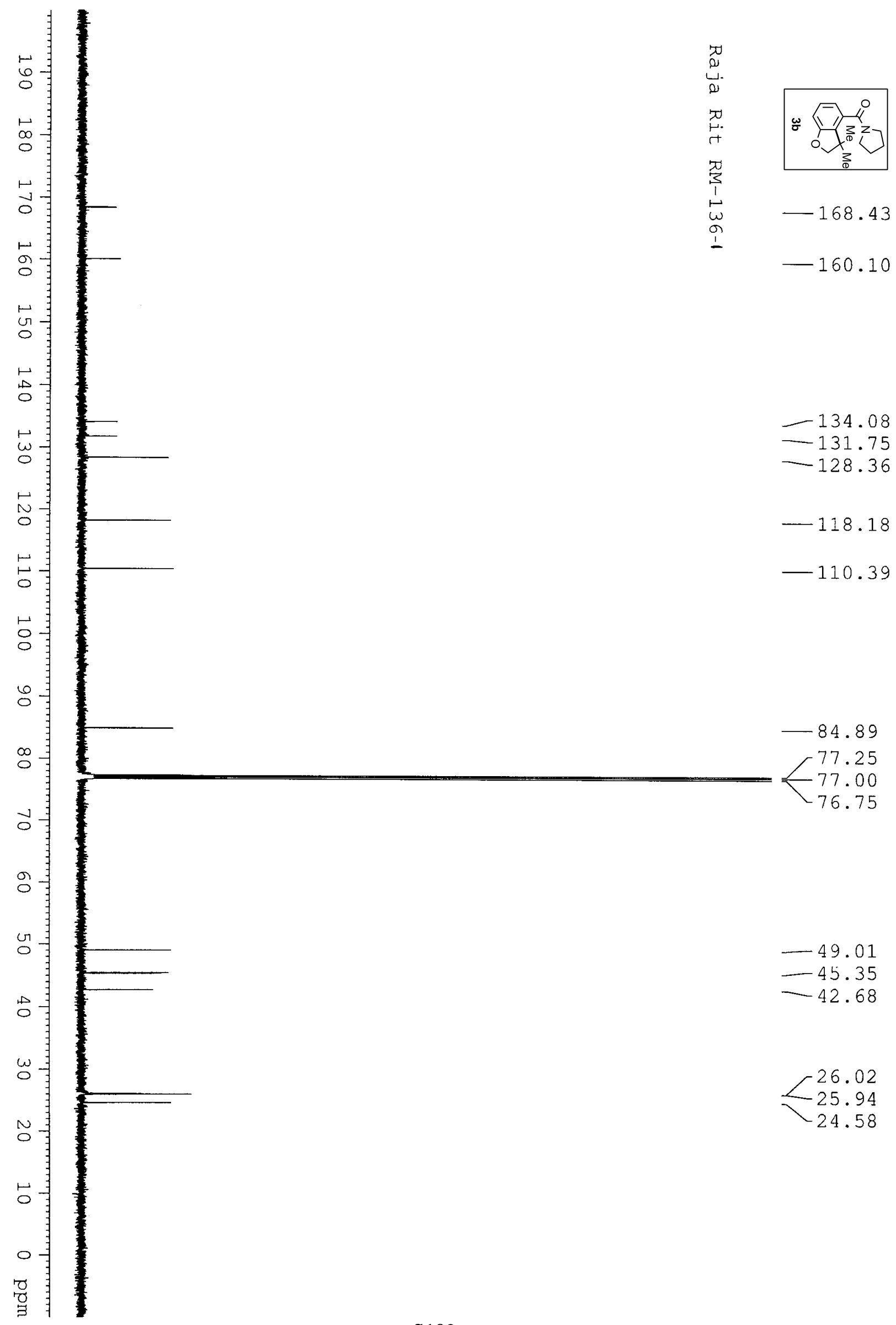

-134.08
-131.75
-128.36

$-118.18$

$-110.39$

$-84.89$

77.25

77.00
-76.75

$-76.75$

$-49.01$

$-45.35$

$-42.68$

26.02

$-25.94$

24.58 


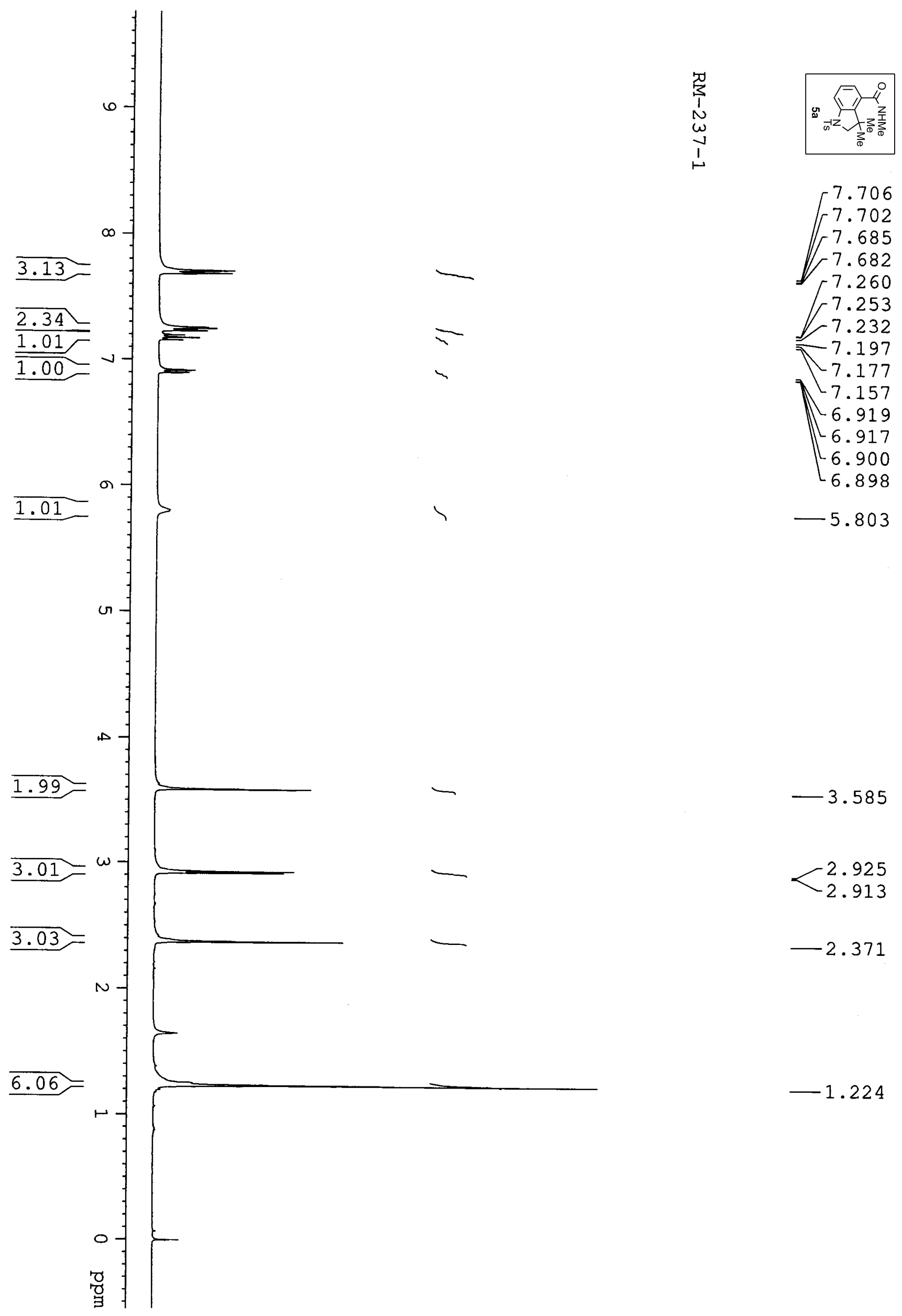



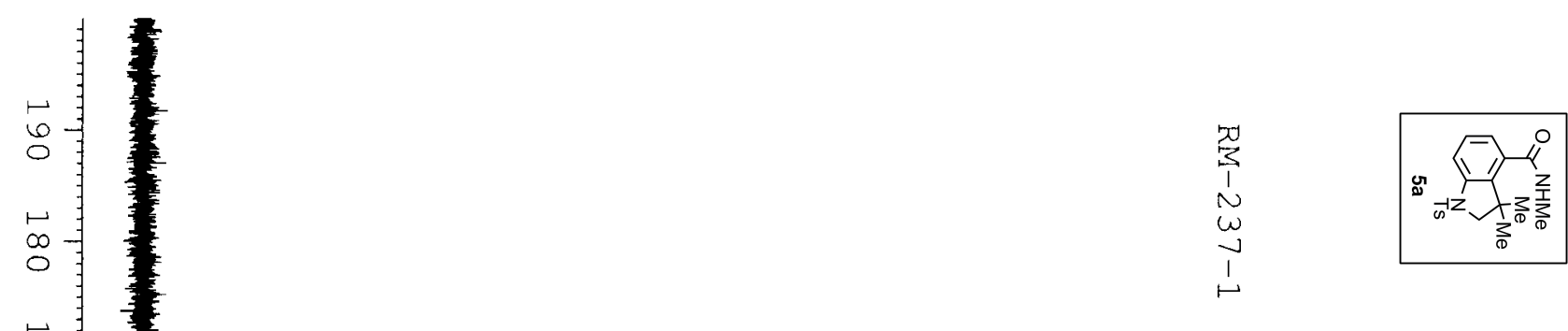

$-169.70$

144.27

$-141.87$

$-137.08$

$-133.96$

$<133.94$

$-129.71$

$-128.08$

127.25

$-122.00$

$-115.81$

卢

훙

$\because$

$\infty$

77.32
-77.00
76.68

$\stackrel{1}{0}$

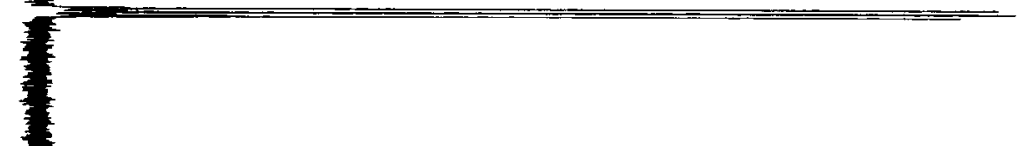

76.68

$-64.51$

용

G

$\stackrel{\circ}{\circ}$

夏

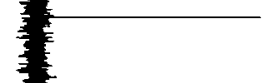

$\omega$

N

。

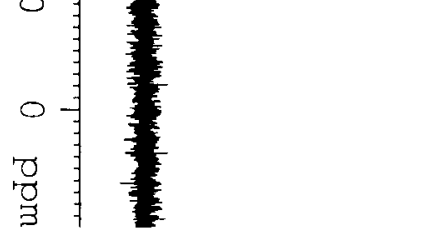

$-41.26$

$<26.61$

26.46

$-21.52$ 


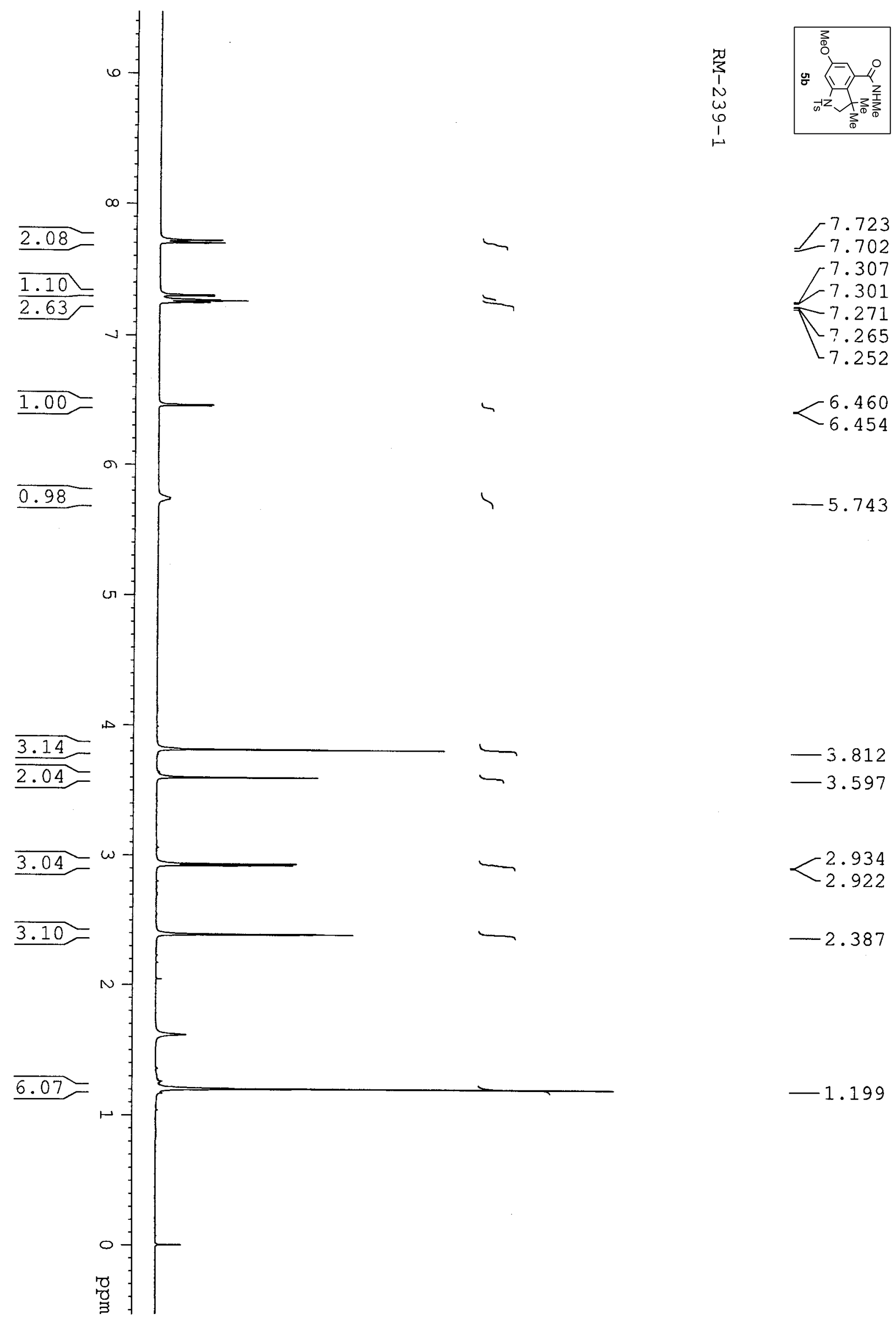



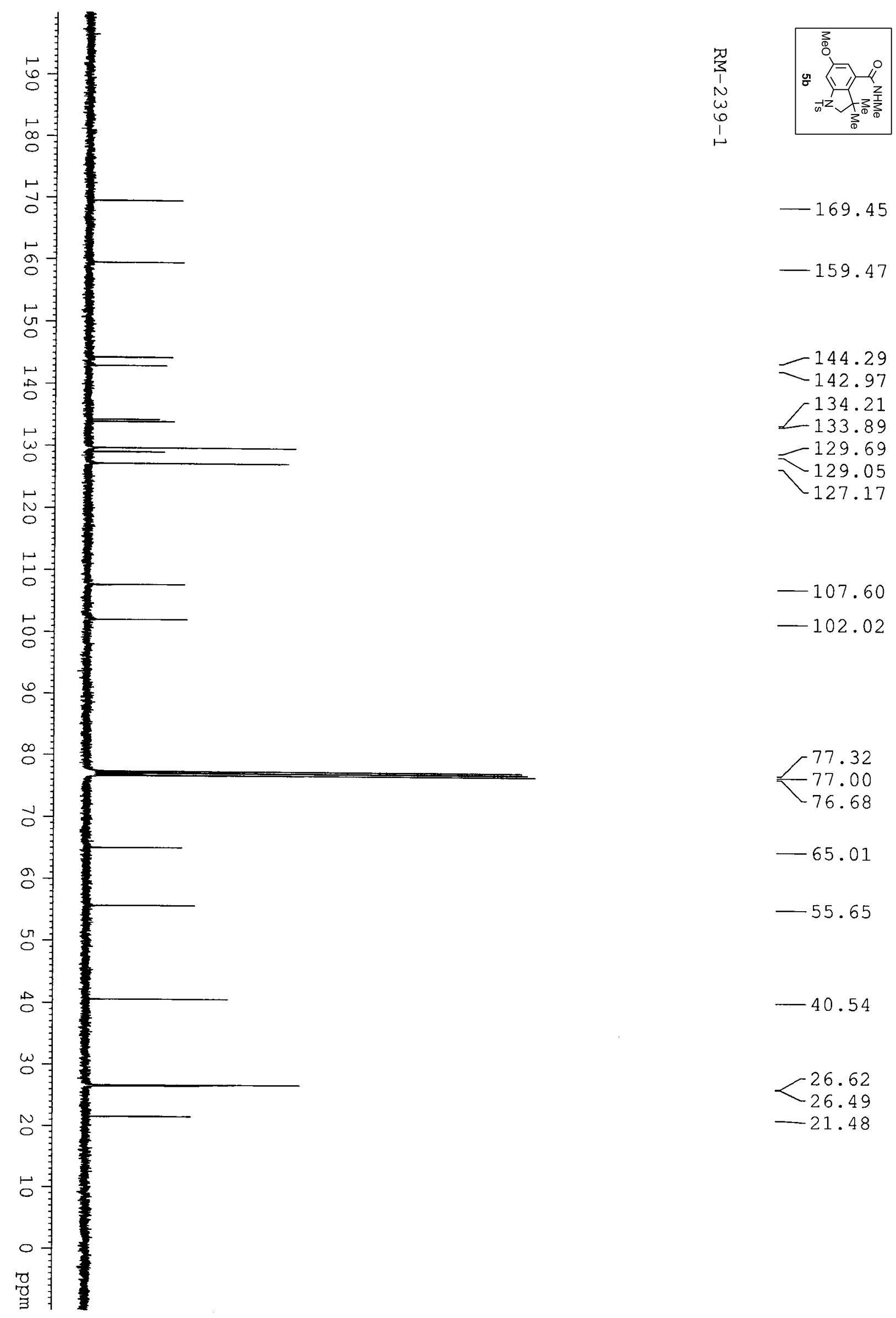

$-144.29$

$-142.97$ 134.21

133.89
-129.69

$-129.69$

$>129.05$

127.17

$-107.60$

$-102.02$

77.32
-77.00
76.68

$-65.01$

$-55.65$

$-40.54$

$<26.62$

$\begin{array}{r}26.49 \\ \hline\end{array}$

$-21.48$ 

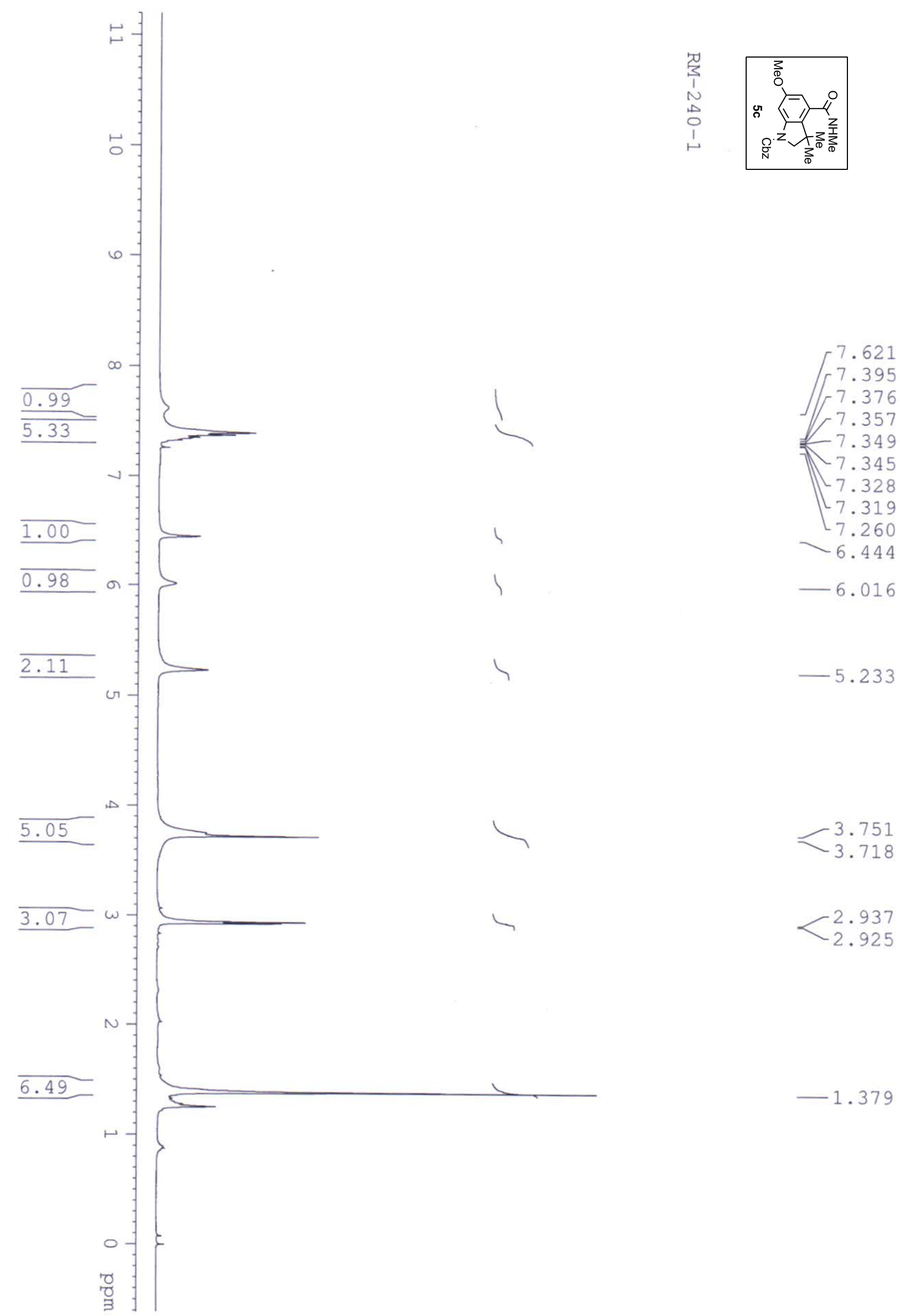

3.751
-3.718

$-2.937$

2.925

$-1.379$ 


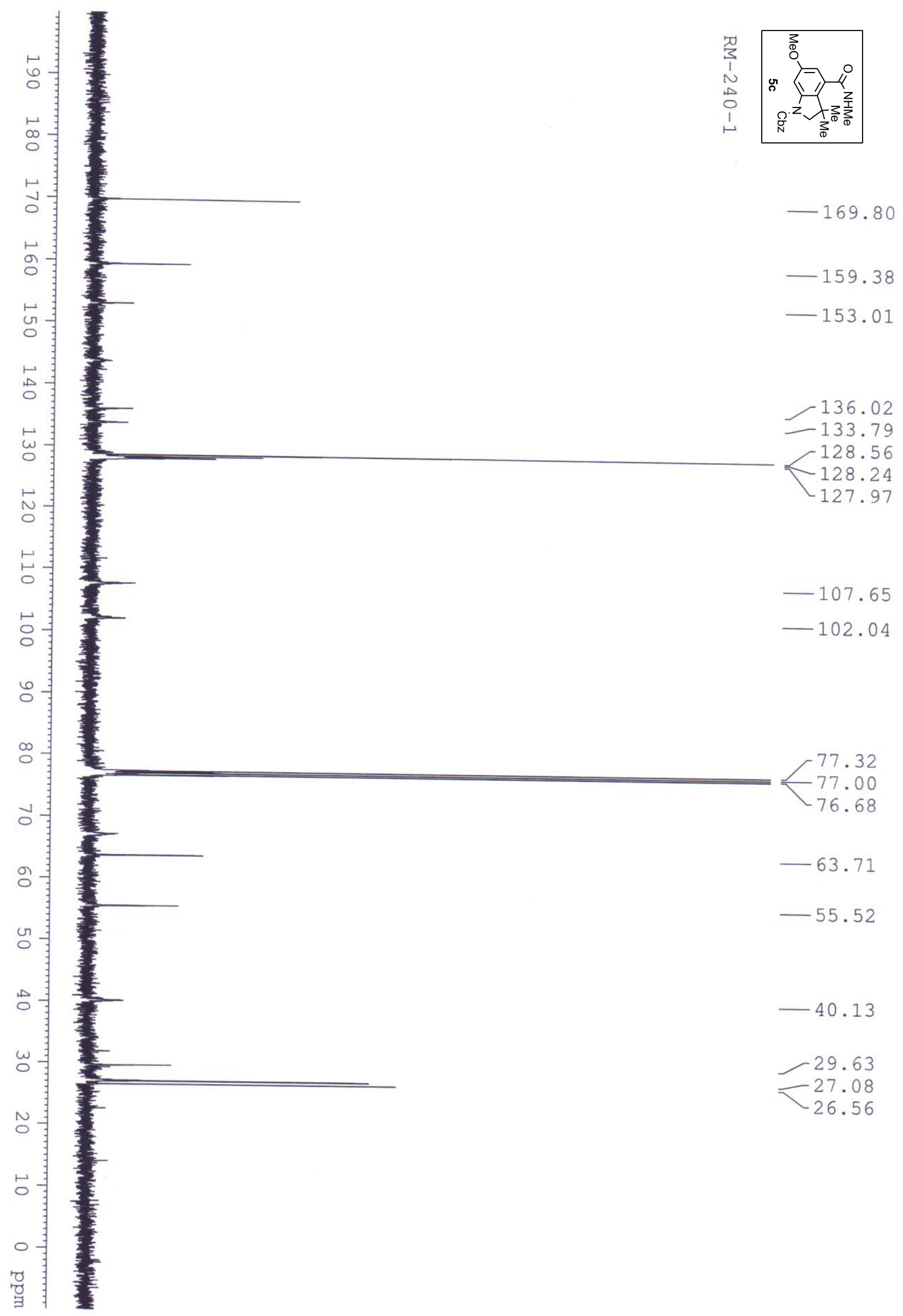




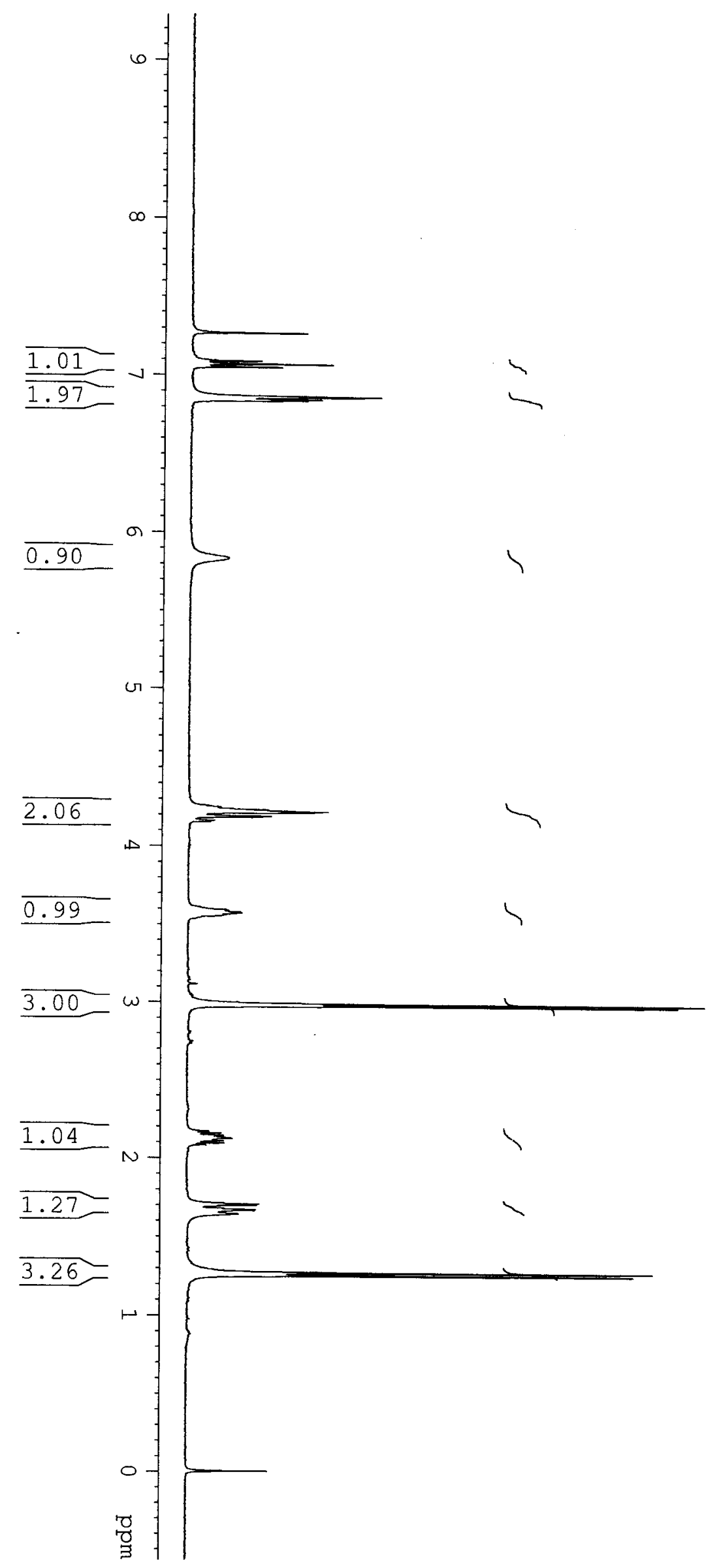

0
3
1
$\omega$
0
$\vdots$
1
1

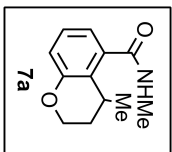

7.264

7.089

$-7.070$

$-7.050$

$-6.863$

$-6.859$

$-6.845$

6.838

$-5.835$

4.245

4.229

4.217

4.211

4.189

4.183

4.161

$-4.155$

3.591

3.585

$-3.573$

$-3.560$

r.986

2.974
2.168

2.155

2.141

$-2.133$

$-2.127$

$-2.121$

$-2.114$

$-2.106$

$-2.092$

2.079

$-1.711$

1.705

1.698

1.691

1.676

1.670

1.663

$-1.656$

1.268

L. 1.250 


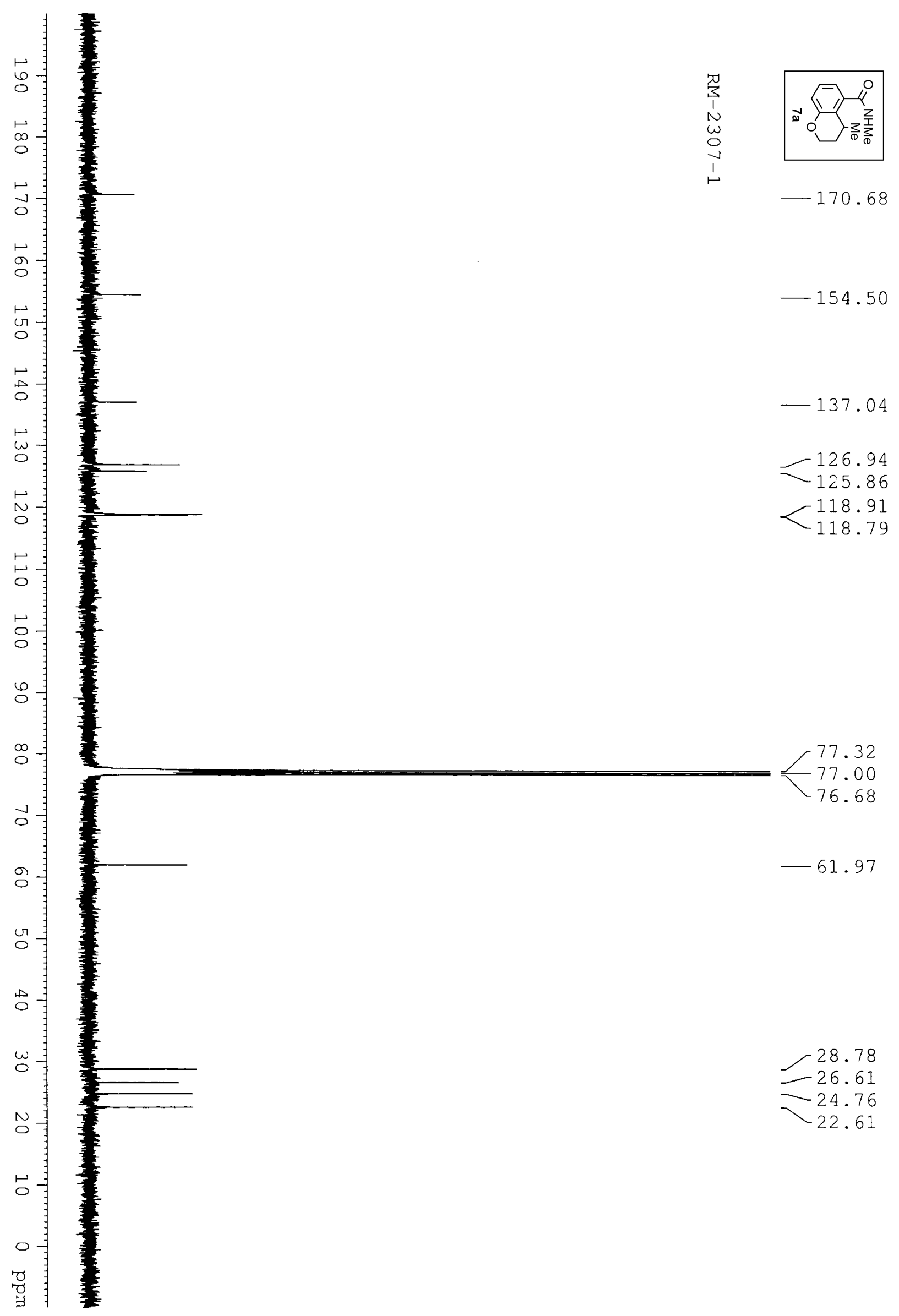




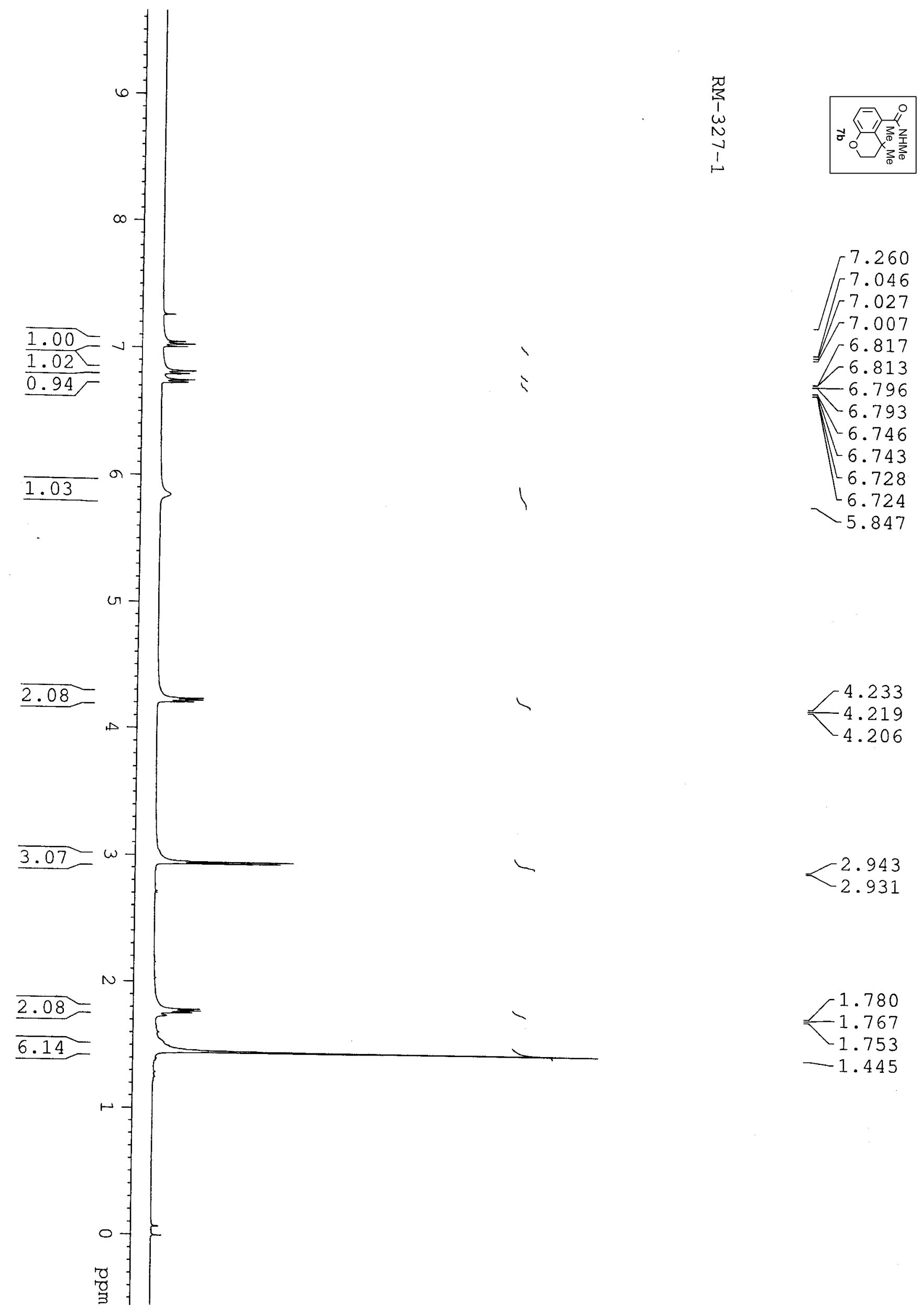



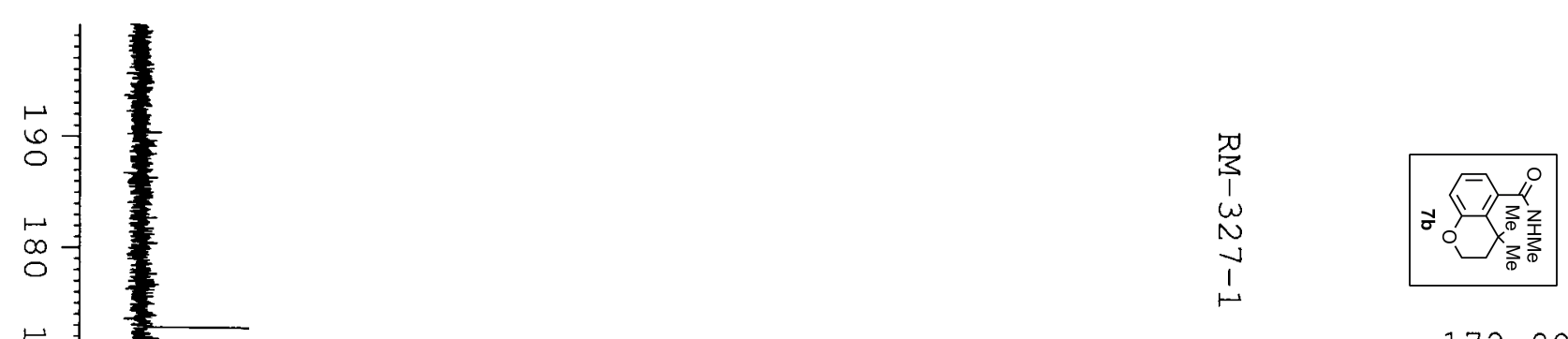

$-172.82$

o

เே

$\circ$

ज

$\stackrel{\vec{\Delta}}{\circ}$



N

艺

○

$\stackrel{5}{\circ}$

$\%$

$\stackrel{\infty}{\circ}$

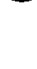

Јै

o青章

G

$\vec{\circ}$



ऽ

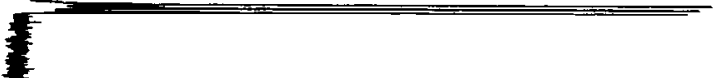

$-154.09$

$-138.03$

$-128.25$

$-126.96$

$-120.11$

$-118.88$

:

$=$

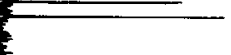

77.32

77.00

$-76.68$

$-62.42$

$-39.42$

$-31.53$

$-29.23$

$-26.74$

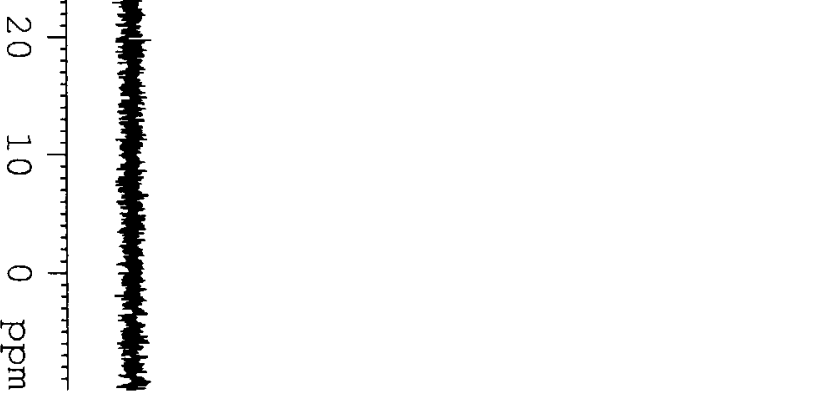




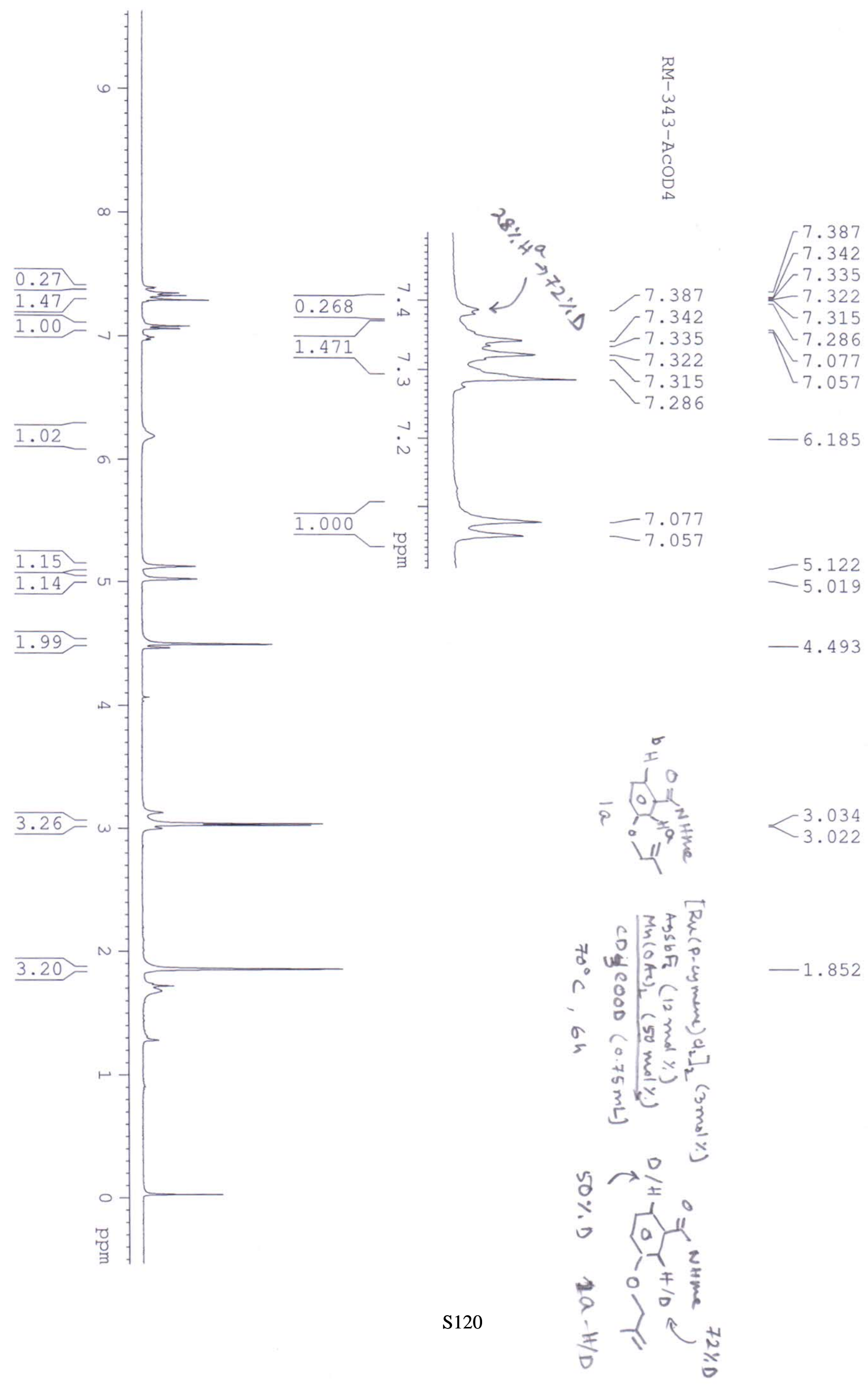




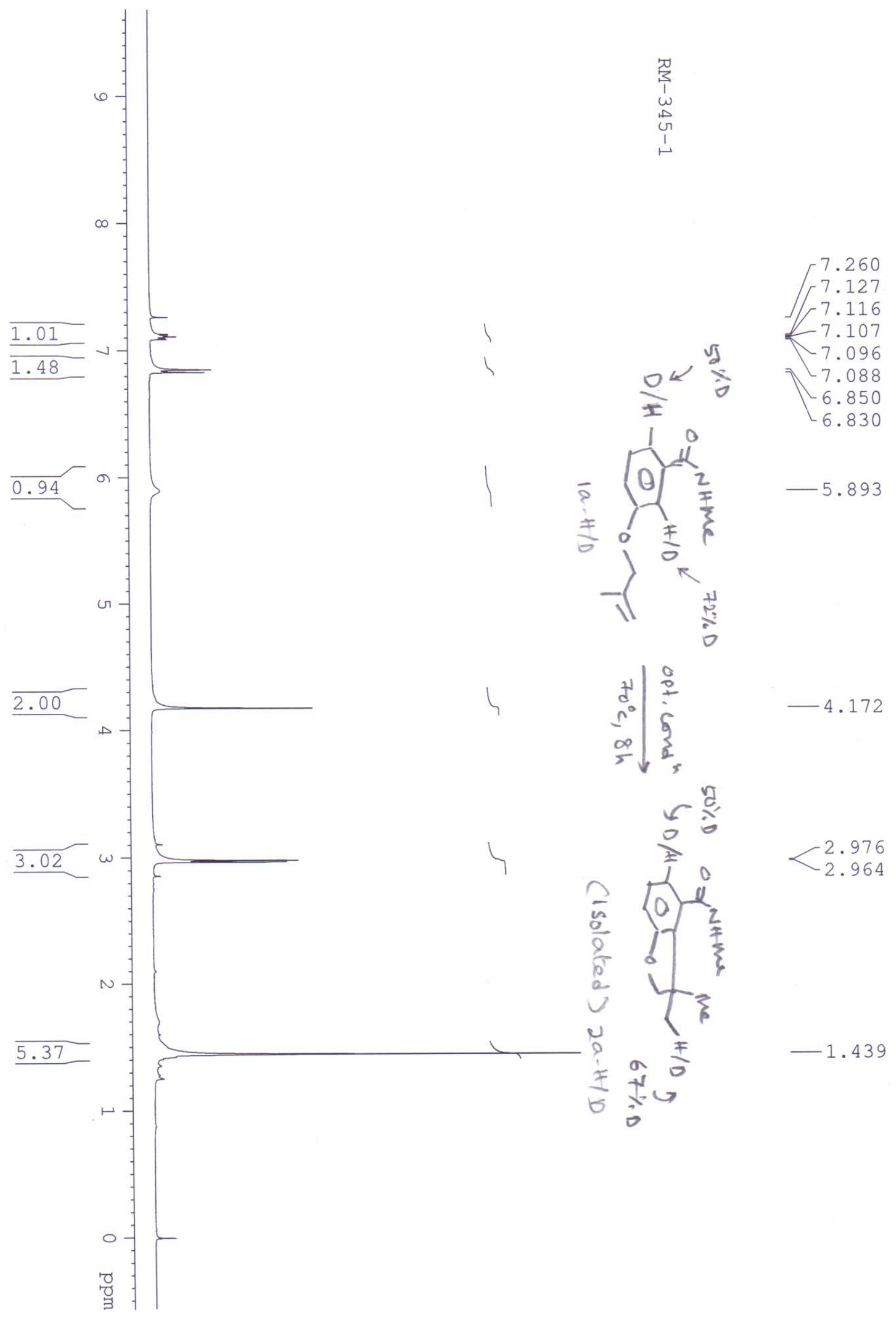




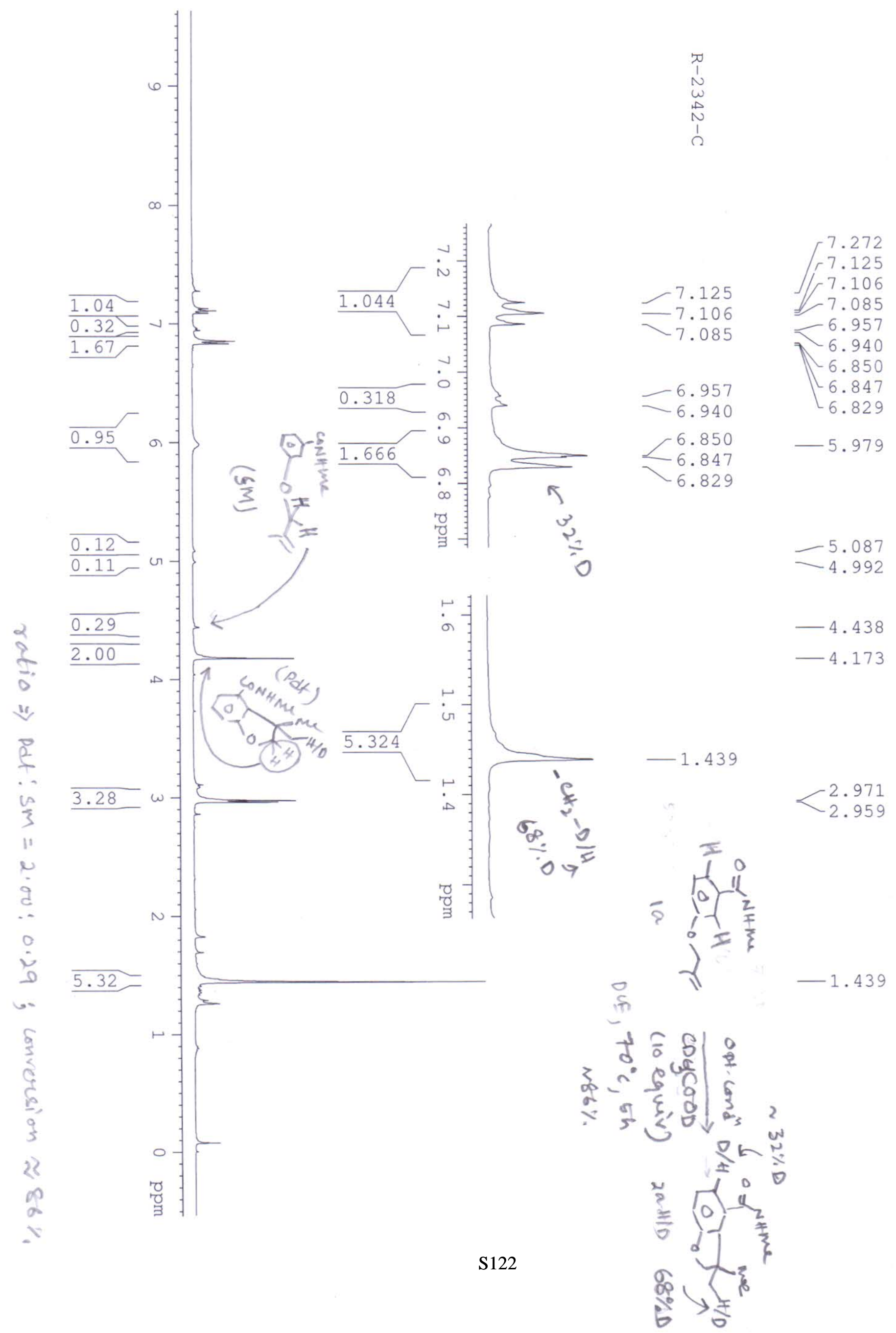


C. Hammett analysis
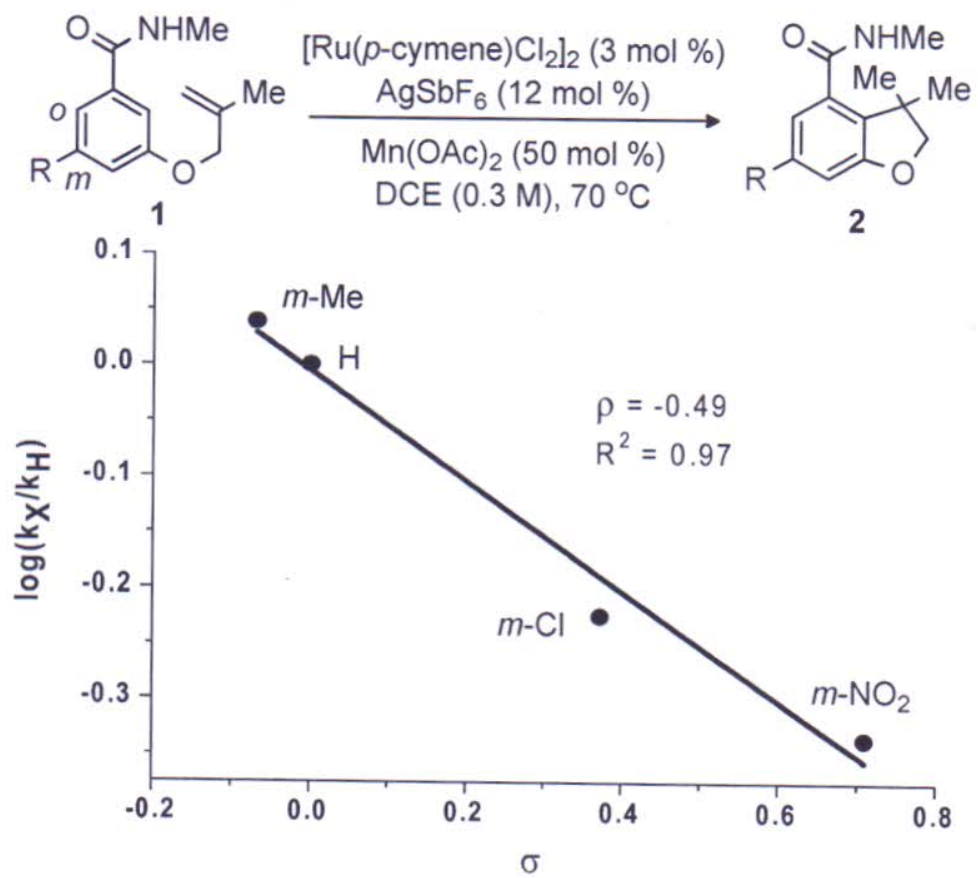

$$
\begin{aligned}
& \log \frac{k_{M e}}{k_{H}}=\log \frac{0.040}{0.0366}=0.0385 \\
& \log \frac{k_{l l}}{k_{H}}=\log \frac{0.0207}{0.0366}=-0.2475 \\
& \log \frac{k_{N D_{2}}}{k_{H}}=\log \frac{0.0169}{0.0366}=-0.3356
\end{aligned}
$$

\begin{tabular}{|c|c|c|}
\hline Entry & $R$ & $\mathrm{~K}_{x}$ \\
\hline 1 & $\mathrm{Me}$ & $0.040 / \mathrm{min}$ \\
2 & $\mathrm{H}$ & $0.0366 / \mathrm{min}$ \\
3 & $\mathrm{Cl}$ & $0.0207 / \mathrm{min}$ \\
4 & $\mathrm{NO}_{2}$ & $0.0169 / \mathrm{min}$ \\
\hline
\end{tabular}

$$
\begin{aligned}
& \sigma_{m-M_{e}}=-0.069 \\
& \sigma_{m-C l}=+0.373 \\
& \sigma_{m-N_{2}}=+0.710
\end{aligned}
$$

* Hammett equation:

$$
\log \frac{k_{x}}{k_{1 A}}=\sigma \cdot \rho
$$

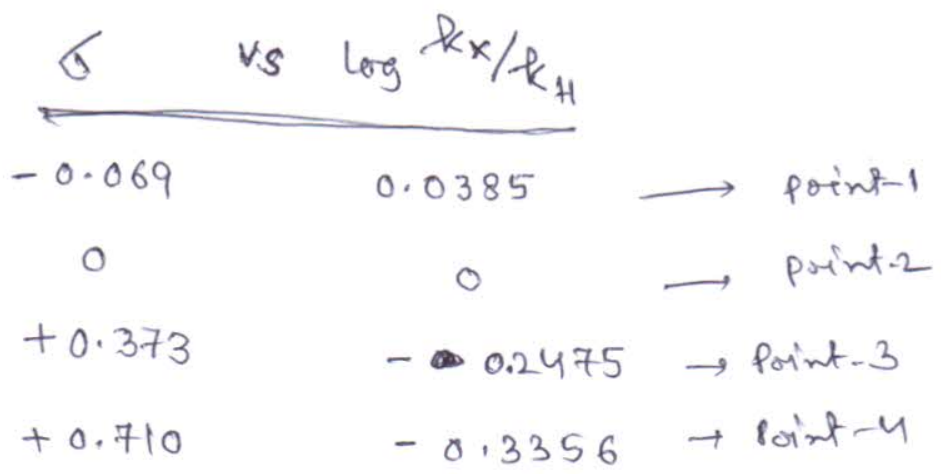

S123 


\section{UOH -SCHOOL OF CHEMISTRY -HRMS}

Analysis Info

Analysis Name

Method

Sample Name

Comment
D:IDatal016IDR.A.K.SAHOOJULYKG-2.d

tune low.m

KG-2-MEOH
Acquisition Date $\quad 7 / 18 / 2016$ 12:07:10 PM

Operator Rajesh Vashisth

Instrument maXis

\begin{tabular}{llllll}
\hline Acquisition Parameter & & & & \\
Source Type & ESI & Ion Polarity & Positive & Set Nebulizer & 0.3 Bar \\
Focus & Not active & Set Capillary & $2500 \mathrm{~V}$ & Set Dry Heater & $180^{\circ} \mathrm{C}$ \\
Scan Begin & $50 \mathrm{~m} / \mathrm{z}$ & Set End Plate Offset & $-500 \mathrm{~V}$ & Set Dry Gas & $4.0 \mathrm{l} / \mathrm{min}$ \\
Scan End & $1500 \mathrm{~m} / \mathrm{z}$ & Set Collision Cell RF & $300.0 \mathrm{Vpp}$ & Set Divert Valve & Waste \\
\hline
\end{tabular}

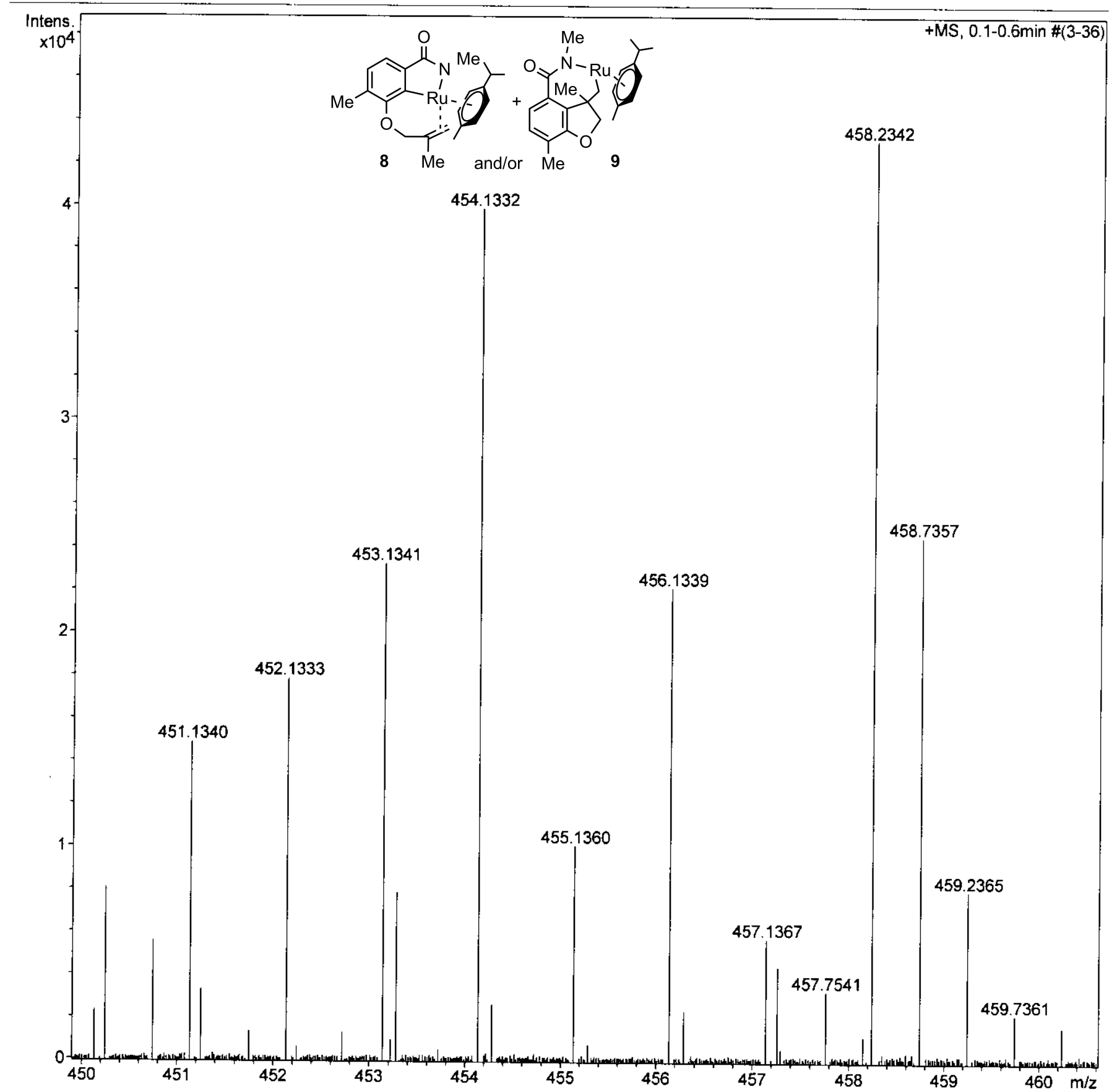

